\title{
Understanding and improving neurofeedback-guided self-regulation
}

Citation for published version (APA):

Skottnik, L. (2020). Understanding and improving neurofeedback-guided self-regulation: On the neuropsychological mechanisms of neurofeedback across mental tasks and time. [Doctoral Thesis, Maastricht University]. Gildeprint Drukkerijen. https://doi.org/10.26481/dis.20200408ls

Document status and date:

Published: 01/01/2020

DOI:

10.26481/dis.20200408ls

Document Version:

Publisher's PDF, also known as Version of record

\section{Please check the document version of this publication:}

- A submitted manuscript is the version of the article upon submission and before peer-review. There can be important differences between the submitted version and the official published version of record.

People interested in the research are advised to contact the author for the final version of the publication, or visit the DOI to the publisher's website.

- The final author version and the galley proof are versions of the publication after peer review.

- The final published version features the final layout of the paper including the volume, issue and page numbers.

Link to publication

\footnotetext{
General rights rights.

- You may freely distribute the URL identifying the publication in the public portal. please follow below link for the End User Agreement:

www.umlib.nl/taverne-license

Take down policy

If you believe that this document breaches copyright please contact us at:

repository@maastrichtuniversity.nl

providing details and we will investigate your claim.
}

Copyright and moral rights for the publications made accessible in the public portal are retained by the authors and/or other copyright owners and it is a condition of accessing publications that users recognise and abide by the legal requirements associated with these

- Users may download and print one copy of any publication from the public portal for the purpose of private study or research.

- You may not further distribute the material or use it for any profit-making activity or commercial gain

If the publication is distributed under the terms of Article $25 \mathrm{fa}$ of the Dutch Copyright Act, indicated by the "Taverne" license above, 


\section{Doctoral Thesis}

\section{Understanding and improving}

neurofeedback-guided self-regulation:

On the neuropsychological mechanisms of neurofeedback across mental tasks and time

Leon Skottnik 
(C) Leon Skottnik, Maastricht 2020.

All rights reserved. No part of this dissertation may be reproduced, stored in a retrieval system, or transmitted in any form, or by any means, electronic, mechanical, photocopying, recording or otherwise, without prior written permission from the author.

Printed by: Gildeprint

Cover: Leon Skottnik

ISBN: 978-94-6380-753-1 


\title{
Understanding and improving
}

neurofeedback-guided self-regulation:

\section{On the neuropsychological mechanisms of neurofeedback across mental tasks and time}

\author{
Dissertation \\ to obtain the degree of Doctor at Maastricht University, \\ on the authority of the Rector Magnificus Prof. Dr. Rianne M. Letschert, \\ in accordance with the decision of the Board of Deans, \\ to be defended in public on Wednesday $8^{\text {th }}$ of April at 14:45 hours \\ by
}

Leon Skottnik 


\section{SUPERVISOR}

Prof. Dr. Rainer Goebel

\section{CO-SUPERVISOR}

Dr. Bettina Sorger

\section{ASSESSMENT COMMITTEE}

Prof. Dr. Alexander T. Sack (chair)

Dr. Florian Krause, Donders Institute for Brain, Cognition and Behaviour, Nijmegen

Dr. Dennis Hernaus

Prof. Dr. Frank Scharnowski, University of Vienna, Austria

The work presented in this dissertation was funded by the European Commission's Health Cooperation Work Programme of the 7th Framework Programme

[Grant Agreement $n^{\circ} 602186$ (BRAINTRAIN)] 


\section{Table of Contents}

1. THE EMBEDDED MIND $\quad 11$

2. DYNAMIC FEEDBACK AND THE BRAIN

3. MEASURING NEURAL PROCESSES FOR BCIS

4. PROVIDING FEEDBACK BASED ON MARKERS OF NEURAL ACTIVATION 18

5. THE NEURAL BASIS OF NEUROFEEDBACK GUIDED SELF-REGULATION 21

CHAPTER 2 - TWO TASKS ARE BETTER THAN ONE: INITIAL MOTOR-IMAGERY NEUROFEEDBACK CAN INCREASE EFFICACY OF RTFMRI NEUROFEEDBACK-GUIDED EMOTION REGULATION TRAININGS

1. INTRODUCTION

2. Methods AND DeSIGN

3. ANALYSIS $\quad 54$

4. RESULTS $\quad 65$

5. DISCUSSION

SUPPLEMENTARY MATERIAL $\quad 92$

CHAPTER 3 - A STABLE NETWORK THAT ADAPTS FLEXIBLY: WHOLE-BRAIN ACTIVATION AND TARGET-REGION CONNECTIVITY DURING RTFMRI NEUROFEEDBACK ACROSS DIFFERENT MENTAL TASKS AND TIME 101

1. INTRODUCTION 104

2. Methods AND DeSIGN 108

3. ANALYSIS 113

4. RESULTS $\quad 122$

5. DISCUSSION $\quad 138$

SUPPLEMENTARY MATERIAL $\quad 150$ 
1. INTRODUCTION

2. METHODS AND DESIGN

3. ANALYSIS

169

4. RESULTS

5. DISCUSSION

2. GENERAL DISCUSSION 


Chapter 1 - General Introduction 

"For the integrity of the whole is impaired if a man break any link in the unity and coherence of either part or cause."

Marcus Aurelius (Jackson, 1906).

\section{The embedded mind}

This extract from Mark Aurelius' "Meditations" reflects the fundament of classical stoic psychology: Human behaviour can only be evaluated in relation to the natural chain of events, in which the underlying mental processes are embedded (Salles, 2017). In classical stoicism, the human mind was viewed as being driven by the same natural forces which govern the environment (Ritter, Gründer, Gabriel, \& Weber, 1971), and this natural chain of events became the most important variable for evaluating and adapting psychological states in relation to it (Weinkauf, 2001a, 2001b).

Much as stoicism, modern psychology developed from deterministic and materialistic philosophical traditions (Schultz \& Schultz, 2008; Sharples, 1986) and like stoic psychology, many therapeutic methods today analyse and reshape cognitive concepts in order to alter emotional states (Beck, 2011; Forschner, 2018; Gross \& Thompson, 2007). Not surprisingly, modern psychology has been influenced directly by stoicism at several points in history, notably during the development of cognitive therapy (Hoellen, 1986; Hollon et al., 2002; Robertson, 2016). But despite these theoretical parallels between stoicism and contemporary psychology, modern experimental psychology had refrained from understanding 
mental states in relation to a constant chain of external events, during its development towards an empirical science: Instead of trying to analyse dynamic interactions between the mind and its environment, prominently Ernst Heinrich Weber and Gustav Theodor Fechner related momentary samples of psychological events to discrete values of physical variables (Fechner, 1860; Schultz \& Schultz, 2008, pp. 78-84). This "psychophysical" approach allowed researchers to identify consistent relationships between psychological experiences and physical events (as operationalized by Wilhelm Wundt, see Figure 1), thereby receiving wide recognition from the scientific community. Over the following decades, the body of research in psychophysics grew tremendously, creating the basis for the rising field of cognitive psychology, and finally cognitive neuroscience, which turned its focus towards the neural basis of mental processes (Gazzaniga, Ivry, \& Mangun, 2009).

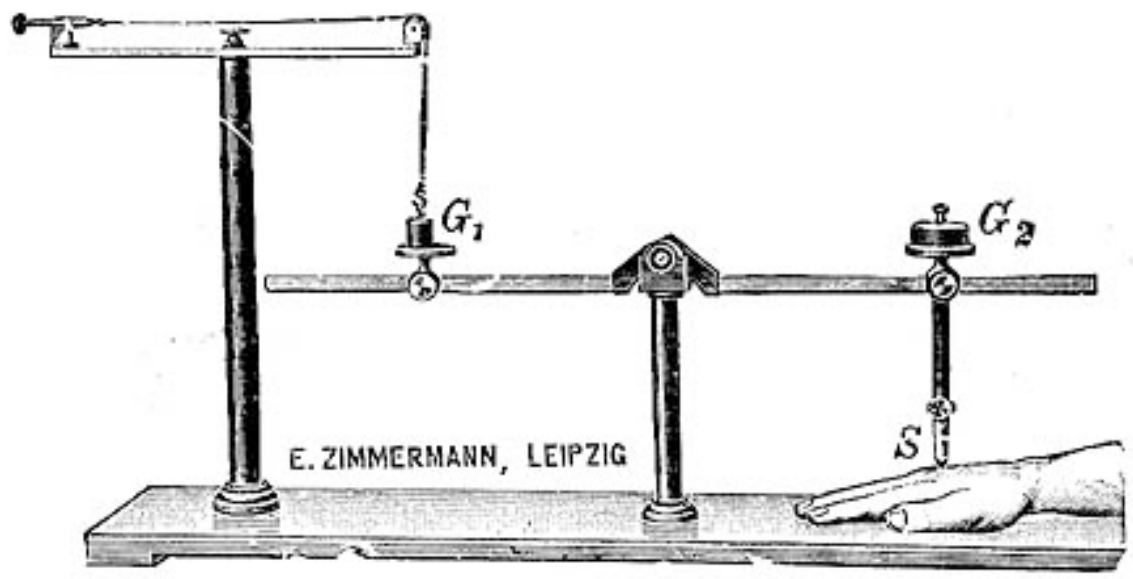

Figure 1: Wilhelm Wundt's aesthesiometer. A measurement device in early psychophysics to study the tactile sensitivity in a controlled fashion, ca. 1883 (Stoelting, 1930).

While during the twentieth century, neuroscientific models for mental processes were emerging rapidly, the majority of experimental protocols were designed to 
detect brain activation associated with predefined stimuli or actions (Maley \& Piccinini, 2016): Participants received for example visual stimulation and according brain activation in visual cortices could be revealed (Blakemore \& Campbell, 1969), or they performed a certain mental action, e.g. mental calculation, and activation in task-related brain regions could be detected (Elul, 1969). Across various domains of neuroscience, these approaches produced extensive knowledge on the neural basis of mental processes, due to their ability to reduce the complexity of the brain by isolating variables of interest. But a disadvantage of these approaches was that the brain was treated as a system which simply reacts to information or creates output (Potter, El Hady, \& Fetz, 2014). Hence, the majority of experimental procedures was tied to a separation of perception, action and subsequent reactions from the environment, thereby ignoring the embodied nature of the brain as a biological system that evolved while interacting with responsive and continuously changing surroundings.

\section{Dynamic feedback and the brain}

How fundamental the ongoing interplay between actions and consequences is implemented in the nervous system, can for example be inferred from the crucial involvement of neural feedback loops in human motor coordination: Ongoing motor behaviour is constantly evaluated and adapted based on dynamically changing feedback information from the visual or somatosensory system (Desmurget et al. (2001), Paillard (1996), see Figure 2 for an early recognition of this issue by René Descartes), even during strongly automatized actions as standing upright (Peterka \& Loughlin, 2004). The importance of dynamic feedback integration is not restricted to processing of basic sensory inputs, but also holds for higher-level cognitive functions, including spoken language (Hickok, Houde, \& 
Rong, 2011; Tourville, Reilly, \& Guenther, 2008) and social cognition (Decety \& Lamm, 2006; Singer \& Lamm, 2009).

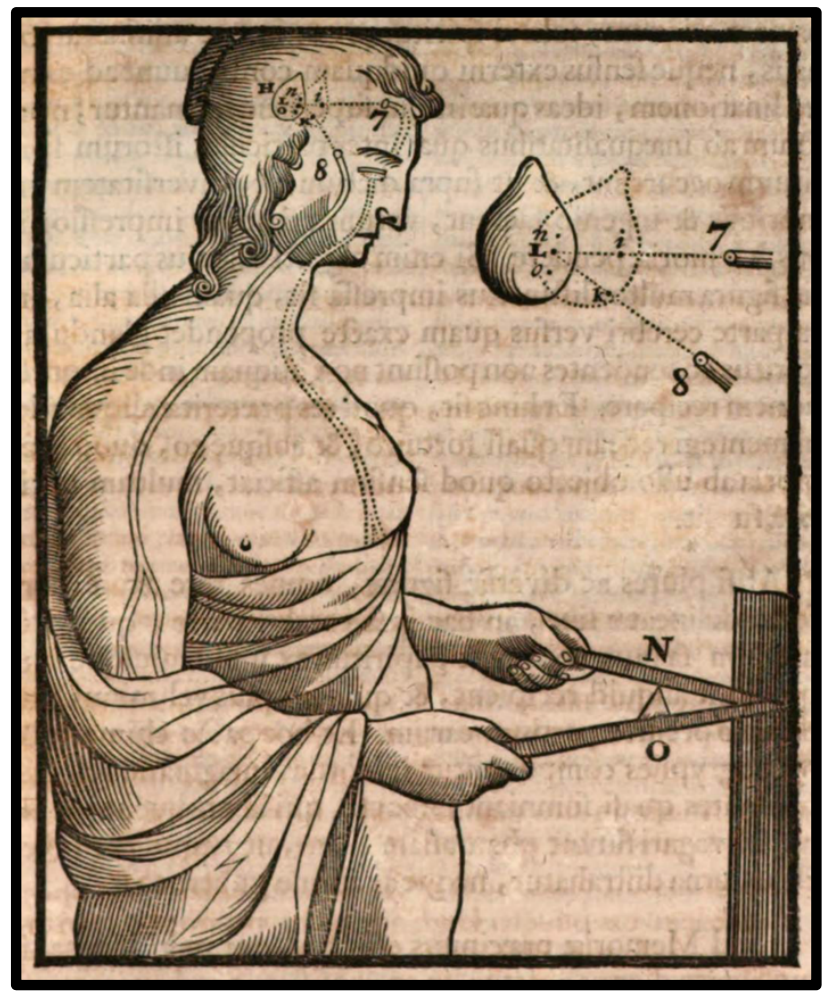

Figure 2: René Descartes' depiction of motor control guided by visual feedback (Descartes, 1677). Multisensory feedback integration forms a basic principle of the human nervous system and is a crucial component of complex motor behaviour.

In experimental settings, dynamic feedback environments were primarily created by detecting changes in behaviour and adapting stimulation accordingly (Mané, Adams, \& Donchin, 1989) or by providing biofeedback based on sympathetic/parasympathetic activity, as for example heart rate (Gatchel, Hatch, Maynard, Turns, \& Taunton-Blackwood, 1979; Goldstein, Ross, \& Brady, 1977; Whitehead, Drescher, Heiman, \& Blackwell, 1977; Williamson \& Blanchard, 1979) 
or electrodermal activity (Crider, 1978; Nagai, Goldstein, Fenwick, \& Trimble, 2004; Toomin \& Toomin, 1975). But technical set-ups which measure and process correlates of neural activation in real-time, also created the possibility to provide feedback from ongoing brain activation using brain-computer interfaces (BCls), which receive input from measurement devices that record markers of neural activity and facilitate this information to control an output device (Figure 3 ).

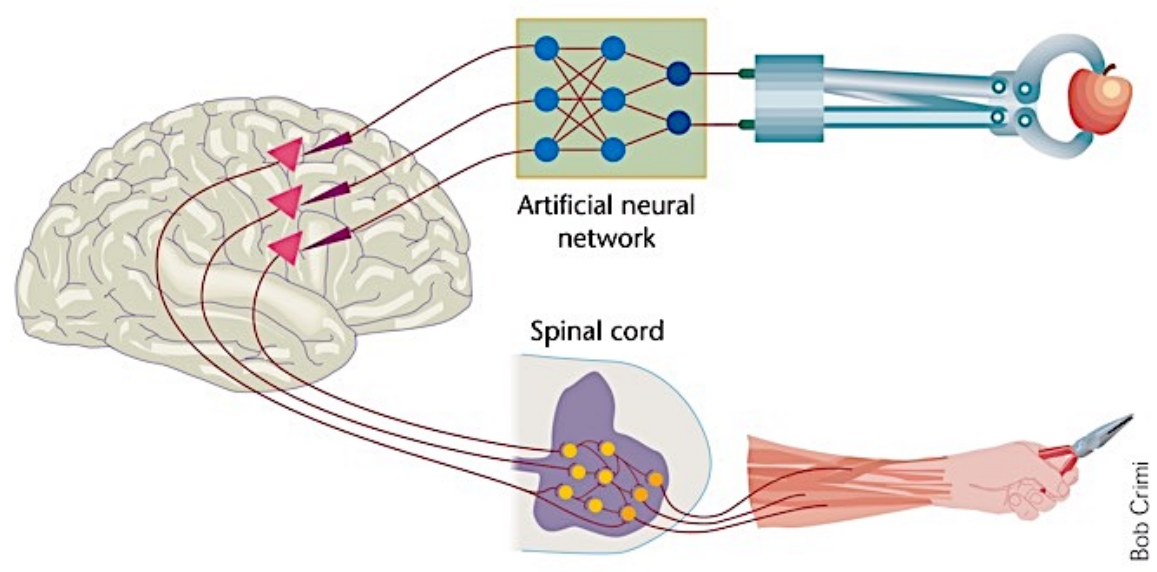

Figure 3: Schematic comparison between a $\mathrm{BCl}$ for motor control and natural motor control. During natural motor control, motor cortex neurons send information to the spinal cord, where it is distributed to contract skeletal muscles. In the depicted $\mathrm{BCl}$, motor cortex activity is recorded and transferred to an external computing device, which recomputes the incoming information using an artificial neural network to invoke movements of a robotic arm, adapted from Fetz (1999).

\section{Measuring neural processes for $\mathrm{BCls}$}

Taking a closer look at the first conceptual stage of a $\mathrm{BCl}$, its input, already reveals the multifaceted possibilities of $\mathrm{BCls:} \mathrm{Up} \mathrm{to} \mathrm{this} \mathrm{date,} \mathrm{BCl}$ s have successfully been created by facilitating information from a variety of neural processing levels, i.e., 
from the cellular level based on activity from single neurons (Chamorro, Levi, Rodríguez, Pinto, \& Varona, 2009; Fetz, 1969; Moritz \& Fetz, 2011) or local field potentials (for an overview see Maling and Mclntyre (2016)), to markers of brain activation extracted from large neural clusters as functional or macro-anatomical regions of interest (e.g. Berman, Horovitz, and Hallett (2013); deCharms et al. (2005); Garrison et al. (2013); Greer, Trujillo, Glover, and Knutson (2014); Haller, Birbaumer, and Veit (2010); Zotev et al. (2011)), including sub-voxel activation pattern within these regions (Shibata, 2012; Shibata, Sasaki, Watanabe, \& Kawato, 2016), activation across whole functional networks (McDonald et al., 2017; Skouras \& Scharnowski, 2018), or indices of inter-regional connectivity (Hwang et al., 2011; Liew et al., 2016; Pineda, Juavinett, \& Datko, 2012; Yamashita, Hayasaka, Kawato, \& Imamizu, 2017).

Given the multitude of techniques that could potentially be used to create feedback from ongoing brain processes, it remains an open issue under which circumstances a specific neural marker is superior to others in certain clinical or research settings. For practical reasons however, non-invasive techniques such as scalp electroencephalography (EEG) and real-time functional magnetic resonance imaging (rtfMRI) have dominated human $\mathrm{BCl}$ applications so far, as they do not require potentially hazardous neurosurgical procedures like intracranial recording techniques (Morshed \& Khan, 2014).

But also $\mathrm{FMRI}$ and EEG gather distinct types of information and are therefore associated with certain restraints with regard to the information that they can provide. EEG records extracellular electrophysiological signals that dominantly reflect synaptic activity (Buzsáki, Anastassiou, \& Koch, 2012). By relying on electrophysiological signals, EEG can track neural processes with a higher temporal 
resolution compared to $\mathrm{fMRI}$, which does not measure neural activity itself, but rather indirect markers of neural activity, i.e. fluctuations in haemoglobin-oxygen bindings in the cerebral blood system (Ogawa, Lee, Kay, \& Tank, 1990; Ogawa et al., 2000; Ogawa \& Lee, 1990). This blood-oxygen-level dependent signal (BOLD signal) measured by fMRI reliably correlates with neuronal activity (Brinker et al., 1999; Mathiesen, Caesar, Akgören, \& Lauritzen, 1998; Ogawa et al., 2000; Rees, Friston, \& Koch, 2000). However, peaks of BOLD signal changes only occur at about 6 - $8 \mathrm{sec}$ after a neural source event due to the sluggishness of the hemodynamic response (i.e. cerebral blood flow changes in response to neural activity; for a comprehensive overview on the relationship between neural activity and the BOLD signal see Logothetis, Pauls, Augath, Trinath, and Oeltermann (2001)). Together with the relatively slow sampling rate applied in fMRI of typically about 2 - 4 sec (Stroman, 2016), the sluggishness of the hemodynamic response constitutes the major cause for the relatively poor temporal resolution of fMRI (Miezin, Maccotta, Ollinger, Petersen, \& Buckner, 2000).

In addition to differences in temporal resolution, $\mathrm{FMRI}$ and EEG differ with regard to their spatial resolution and brain coverage. The spatial resolution of EEG is hampered by the fact, that electrical signals travel through intracranial tissue into various directions: When arriving at the outer scalp skin, electrophysiological signals of interest are heavily scattered and corrupted by physiological noise. This causes a low spatial resolution of about 5 - 9 mm of EEG (Babiloni, Cincotti, Carducci, Rossini, \& Babiloni, 2001; Burle et al., 2015; Nunez et al., 1994). Additionally, this also results in profound limitations with regard to brain coverage, as signals that originate from subcortical and deep temporal areas are too distorted by noise for sensible analysis (Grech et al. (2008); interestingly recent modelling approaches based on combined fMRI-EEG data are nevertheless able to utilize 
activation from the amygdala for BCls (see Keynan et al. (2019), but note that the selected fingerprint might reflect prefrontal activation strongly associated with amygdala activity). In contrast, the spatial resolution of fMRI typically lies around 2 - $4 \mathrm{~mm}$ and activation in subcortical structures can be tracked reliably (Auer \& Auer, 1998). Furthermore, through application of high-field fMRI even sub-millimetre spatial-resolution can be achieved (Duyn, 2012) and high-field rtfMRI neurofeedback BCls have already successfully been implemented (Gröne et al., 2015)

\section{Providing feedback based on markers of neural activation}

After a marker of neural processing has been extracted for a $\mathrm{BCl}$, the retrieved information is facilitated in order to control an output device. If the behaviour of the output device is fed back to the subject, the subject can learn to adapt his own neural activity to gain control over the output device (Velliste, Perel, Spalding, Whitford, and Schwartz (2008), Figure 4), thereby using the behaviour of the output device as feedback on his own brain state.

Taking into account that neural feedback loops form the mechanistic core process of learning and memory formation (notably as driving mechanism of operant conditioning, see Staddon and Cerutti (2003); Adams (1971), Adams, Goetz, and Marshall (1972)), the high potential for research and clinical applications of $\mathrm{BCls}$ becomes evident. $\mathrm{BCl}$ set-ups that focus on providing participants with feedback on their current brain activation in order for them to achieve a certain neural target state, termed neurofeedback paradigms (Figure 5), have therefor been widely used (an up-to-date review on such neurofeedback approaches was recently created by Jeunet, Lotte, Batail, Philip, and Micoulaud Franchi (2018)). 

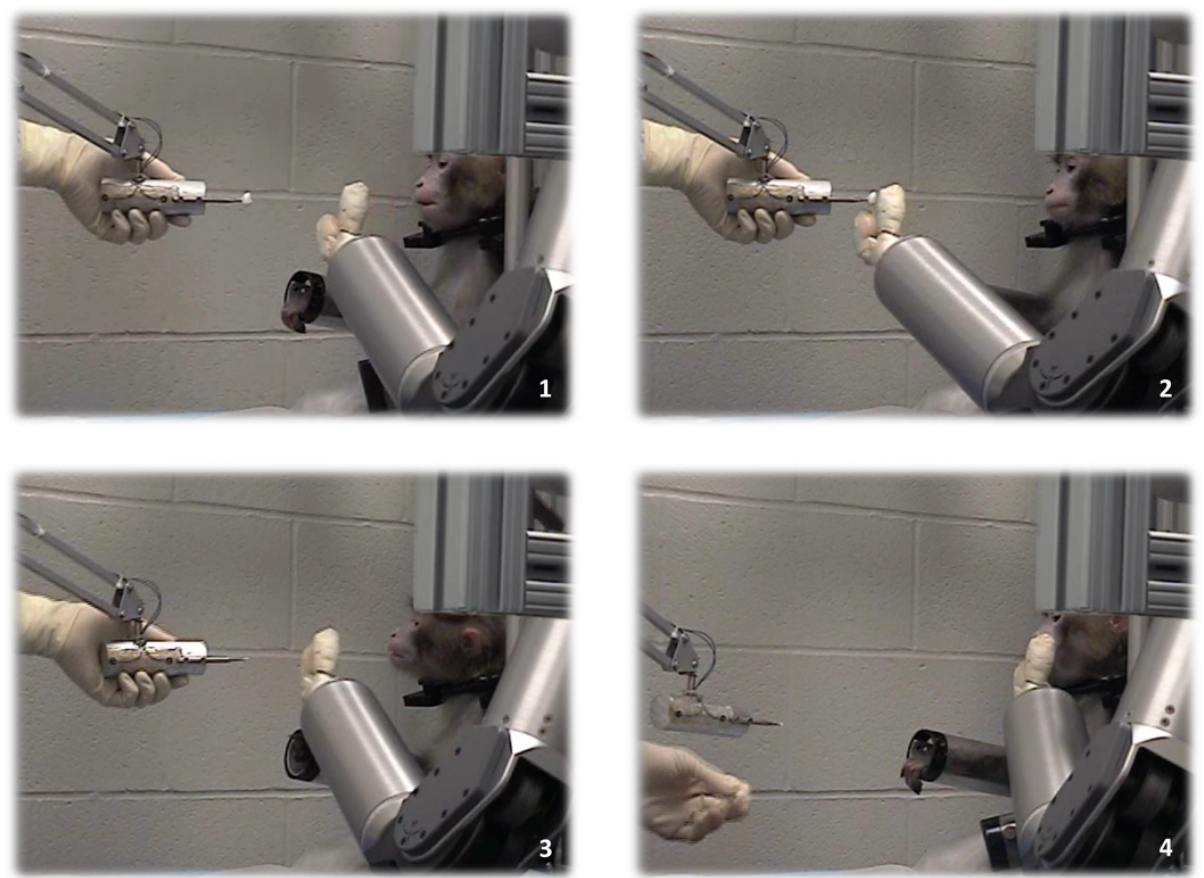

Figure 4: Example of a $\mathbf{B C l}$ for visuomotor control. Neural activity from the motor cortex of a monkey is recorded and facilitated to guide movements of a robotic arm. When food is presented to the monkey, the animal adapts its motor cortex activity to reach for the food using the robotic arm ( 1 and 2 ) and move it back to his mouth ( 3 and 4). Visual feedback about the position of the robotic arm is thereby used by the monkey to guide his motor cortex activity. Velliste et al. (2008), 4:21 (1), 4:22 (2), 4:33 (3), 4:45 (4).

While in humans, early EEG neurofeedback BCls already appeared in 1960 (for a summary of the early history of EEG neurofeedback see Kamiya (2011)), contemporary $\mathrm{BCl}$ applications increasingly apply hemodynamic neuroimaging techniques as rtfMRI (early neurofeedback studies by Yoo and Jolesz (2002)), due to the higher spatial resolution of $\mathrm{fMRI}$ as well as its ability to reliably track 
activation in limbic areas, key structures involved in major psychopathologies as mood disorders (Baumann et al., 1999; Carroll, Curtis, \& Mendels, 1976; Chen, Suckling, Lennox, Ooi, \& Bullmore, 2011), addiction (Everitt, Dickinson, \& Robbins, 2001; Koob, Sanna, \& Bloom, 1998; Makris et al., 2008; Wikler, Norrell, \& Miller, 1972) and anxiety disorders (Modell, Mountz, Curtis, \& Greden, 1989; Shin, Rauch, \& Pitman, 2006; Stein, Goldin, Sareen, Zorrilla, \& Brown, 2002).

So far, rtfMRI neurofeedback paradigms (Figure 5) have most commonly provided participants with sensory (dominantly visual) neurofeedback (Arns et al. (2017); notably recent approaches also deliver neurofeedback to the brain using brain stimulation techniques as transcranial magnetic stimulation, see Sokhadze et al. (2014), Koganemaru et al. (2018)). By receiving visual neurofeedback, participants could learn to gain control over clinically relevant neural target processes as brain activation related to affective states (Johnston, Boehm, Healy, Goebel, \& Linden, 2010). By providing patients with the possibility to undergo neurofeedback guided self-regulation trainings, previous studies successfully showed that rtfMRI neurofeedback could elevate the psychiatric and neural symptomatology of patients with the most prevalent mental disorders in Europe (Alonso et al., 2004), i.e. mood disorders (Linden et al., 2012; Young, Misaki, et al., 2017; Young, Siegle, et al., 2017; Yuan et al., 2014), addiction (Canterberry et al., 2013; Hanlon et al., 2013; Kim, Yoo, Tegethoff, Meinlschmidt, \& Lee, 2015; Li et al., 2013) and anxiety disorders (Gerin et al., 2016; Nicholson et al., 2017; Scheinost et al., 2013; Zilverstand, Sorger, Sarkheil, \& Goebel, 2015). 


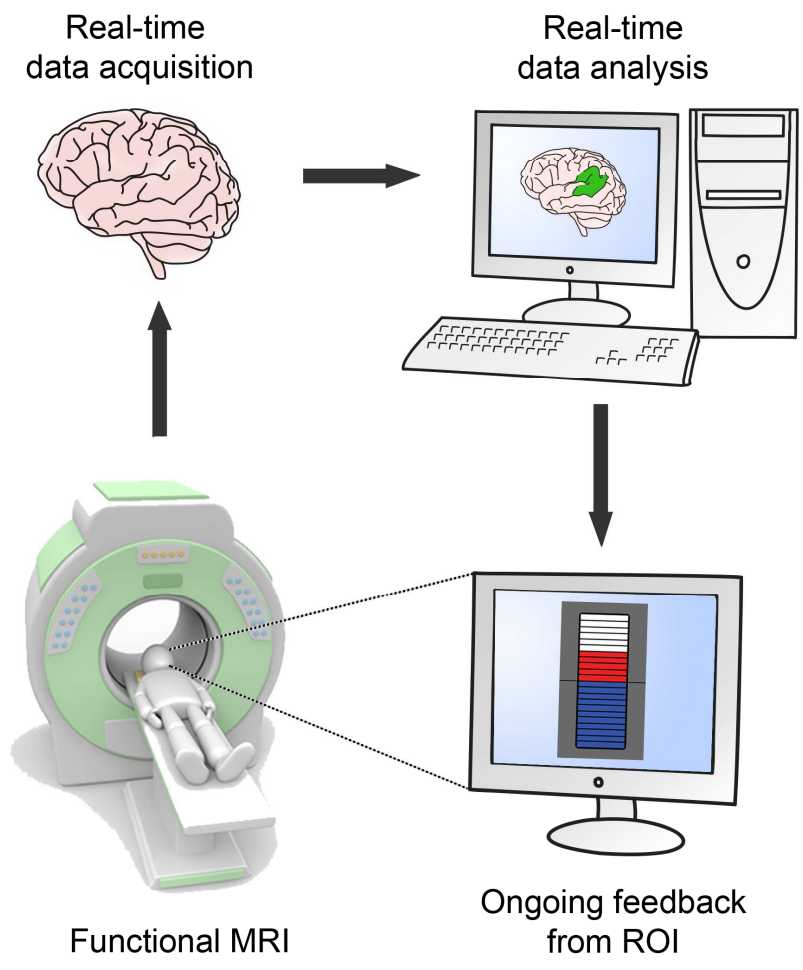

Figure 5: A common fMRI neurofeedback set-up. Changes in the blood-oxygen-level dependent signal are measured and processed in real-time. The mean signal change of a region of interest (ROI) is presented visually to the participant by colouring segments of a thermometer to indicate the magnitude of regional brain activation (Thibault, MacPherson, Lifshitz, Roth, \& Raz, 2017).

\section{The neural basis of neurofeedback guided self-regulation}

But despite decades of applying neurofeedback in neuroscience, psychology and medicine, considerably little is known about the neural processes involved in neurofeedback-guided self-regulation. Although previous research suggests an interplay of reward processing, self-regulation and learning mechanisms in interaction with brain networks involved in the specific mental task driving the 
feedback (Sitaram et al., 2017), studies that investigate underlying neural mechanisms of neurofeedback are sparse: While a recent meta-analysis reported extensive overlap of whole-brain activation across different neurofeedback studies in prefrontal, parietal, occipital as well as subcortical areas and deactivation of the default-mode-network (Emmert et al., 2016), this study did not control for general effects of self-regulation. Studies that aimed to disentangle the neural basis of neurofeedback in turn from the neural basis self-regulation itself, have done so only for very specific self-regulation tasks, e.g. for motor imagery (Marchesotti et al. (2017)).

So far, theoretical accounts on the neural basis of neurofeedback had therefor to rely on accumulated evidence from different single studies with considerable variability in applied methods and study contents. In addition to the scarceness of structured evidence on the neural basis of neurofeedback guided self-regulation, the field also lacks evidence on the temporal properties of the neural processes that take place within involved networks. Taking into account how crucial timing is in operant conditioning (Grossberg, 1971; Staddon \& Cerutti, 2003), these questions appear to be fundamental for the understanding how neurofeedback training can induce learning and reshape the brain.

By examining the three main neuropsychological processes underlying neurofeedback training (Sitaram et al., 2017), i.e., feedback processing, selfregulation and learning, the work presented in this thesis addresses open issues concerning the functional basis of neurofeedback trainings and ultimately strives to underline the practical relevance of these issues for the design of future neurofeedback interventions. Evidence on the neural and psychological basis of 
neurofeedback was collected to provide an overview on how humans learn to gain control over provided neurofeedback signals in the following studies:

\section{Chapter 2}

The work presented in chapter two of this thesis focusses on effects of neurofeedback interventions that are modulated by differences in training styles. Specifically, the effect of providing an initial neurofeedback task with assumed lower task demands before switching to the main training was investigated. Ten participants underwent three rtfMRI neurofeedback sessions, in order to learn to regulate activation of the anterior insula (aINS) to three different target levels, either by performing the identical emotion regulation task over the whole training, or by first training to reach three target levels with motor-imagery-driven neurofeedback from the supplementary motor area (SMA). After evaluating the task difficulty of SMA and aINS neurofeedback, effects of the two training styles were compared with regard to self-regulation behaviour, self-regulation performance and psychological measures associated with positive learning outcomes. By connecting the gathered findings, this chapter aims to evaluate whether combining different neurofeedback tasks with varying task difficulty can improve the learning process during neurofeedback interventions.

\section{Chapter 3}

The work presented in chapter three of this thesis focusses on the neural basis of self-regulation during neurofeedback across different neurofeedback tasks and training time-points. Whole-brain activation and connectivity of the neurofeedback target regions to the whole brain in the participants undergoing the procedure described in chapter 2 are analysed. This chapter thereby aims to disentangle task general brain networks from tasks specific networks that become active during 
neurofeedback guided self-regulation. In order to observe how the neural basis of self-regulation changes during neurofeedback over time, activation and connectivity were additionally compared between the different training sessions over the course of the whole three week intervention.

\section{Chapter 4}

The research presented in chapter four of this thesis focusses on the neural basis of feedback processing during neurofeedback. To contribute to an understanding of neurofeedback that holds value across different mental task domains, neurofeedback guided self-regulation was compared to self-regulation without neurofeedback across various mental tasks. Ten participants freely chose one selfregulation task and underwent two training sessions during fMRI scanning, one with and one without receiving rtfMRI neurofeedback. During neurofeedback sessions, neurofeedback was provided based on activity in task-related, individually defined target regions. In both sessions, participants aimed at reaching and holding low, medium, or high brain-activation levels in the target region. To investigate the neural mechanisms specific to neurofeedback but independent from general effects of self-regulation, we compared whole-brain activation as measured with functional magnetic resonance imaging (fMRI) across different mental tasks involving gradual self-regulation with and without providing neurofeedback. Furthermore, activation in the striatum, a key region in feedback learning, was analysed and related to self-regulation performance, in order to detect effects specifically modulated by the information contained in the neurofeedback. 


\section{References}

Adams, J. A. (1971). A Closed-Loop Theory of Motor Learning. Journal of Motor Behavior, 3(2), 111-150.

Adams, J. A., Goetz, E. T., \& Marshall, P. H. (1972). Response feedback and motor learning. Journal of Experimental Psychology, 92(3), 391.

Alonso, J., Angermeyer, M., Bernert, S., Bruffaerts, R., Brugha, T., Bryson, H., . . . Demyttenaere, K. (2004). Prevalence of mental disorders in Europe: results from the European Study of the Epidemiology of Mental Disorders (ESEMeD) project. Acta psychiatrica scandinavica, 109, 21-27.

Arns, M., Batail, J.-M., Bioulac, S., Congedo, M., Daudet, C., Drapier, D., . . L Lotte, F. (2017). Neurofeedback: One of today's techniques in psychiatry? L'Encéphale, 43(2), 135-145.

Auer, D., \& Auer, L. (1998). Functional magnetic resonance imaging in clinical neuroscience: Current state and future prospects Minimally Invasive Techniques for Neurosurgery (pp. 237-242): Springer.

Babiloni, F., Cincotti, F., Carducci, F., Rossini, P. M., \& Babiloni, C. (2001). Spatial enhancement of EEG data by surface Laplacian estimation: the use of magnetic resonance imaging-based head models: Elsevier.

Baumann, B., Danos, P., Krell, D., Diekmann, S., Leschinger, A., Stauch, R., . . . Bogerts, B. (1999). Reduced volume of limbic system-affiliated basal ganglia in mood disorders: preliminary data from a postmortem study. The Journal of neuropsychiatry and clinical neurosciences, 11(1), 71-78.

Beck, J. S. (2011). Cognitive behavior therapy: Basics and beyond: Guilford press.

Berman, B. D., Horovitz, S. G., \& Hallett, M. (2013). Modulation of functionally localized right insular cortex activity using real-time fMRI-based neurofeedback. Frontiers in human neuroscience, 7, 638.

Blakemore, C., \& Campbell, F. W. (1969). On the existence of neurones in the human 
visual system selectively sensitive to the orientation and size of retinal images. The Journal of physiology, 203(1), 237-260.

Brinker, G., Bock, C., Busch, E., Krep, H., Hossmann, K. A., \& Hoehn-Berlage, M. (1999). Simultaneous recording of evoked potentials and T-weighted MR images during somatosensory stimulation of rat. Magnetic Resonance in Medicine: An Official Journal of the International Society for Magnetic Resonance in Medicine, 41(3), 469-473.

Burle, B., Spieser, L., Roger, C., Casini, L., Hasbroucq, T., \& Vidal, F. (2015). Spatial and temporal resolutions of EEG: Is it really black and white? A scalp current density view. International Journal of Psychophysiology, 97(3), 210-220.

Buzsáki, G., Anastassiou, C. A., \& Koch, C. (2012). The origin of extracellular fields and currents-EEG, ECoG, LFP and spikes. Nature reviews neuroscience, 13(6), 407.

Canterberry, M., Hanlon, C. A., Hartwell, K. J., Li, X., Owens, M., LeMatty, T., . . . Brady, K. T. (2013). Sustained reduction of nicotine craving with real-time neurofeedback: exploring the role of severity of dependence. Nicotine \& tobacco research, 15(12), 2120-2124.

Carroll, B. J., Curtis, G. C., \& Mendels, J. (1976). Neuroendocrine regulation in depression: I. Limbic system-adrenocortical dysfunction. Archives of General Psychiatry, 33(9), 1039-1044.

Chamorro, P., Levi, R., Rodríguez, F. B., Pinto, R. D., \& Varona, P. (2009). Real-time activity-dependent drug microinjection. BMC Neuroscience, 10(1), P296.

Chen, C. H., Suckling, J., Lennox, B. R., Ooi, C., \& Bullmore, E. T. (2011). A quantitative meta-analysis of fMRI studies in bipolar disorder. Bipolar disorders, 13(1), 1-15.

Crider, A. (1978). The electrodermal response: biofeedback and individual difference studies. Applied Psychology, 28(1), 37-48. 
Decety, J., \& Lamm, C. (2006). Human empathy through the lens of social neuroscience. The scientific World journal, 6, 1146-1163.

deCharms, R. C., Maeda, F., Glover, G. H., Ludlow, D., Pauly, J. M., Soneji, D., . . . Mackey, S. C. (2005). Control over brain activation and pain learned by using real-time functional MRI. Proceedings of the National Academy of Sciences of the United States of America, 102(51), 18626-18631.

Descartes, R. (1677). Tractatus de homine, et de formatione foetus: Elsevir.

Desmurget, M., Gréa, H., Grethe, J. S., Prablanc, C., Alexander, G. E., \& Grafton, S. T. (2001). Functional anatomy of nonvisual feedback loops during reaching: a positron emission tomography study. Journal of Neuroscience, 21(8), 29192928.

Duyn, J. H. (2012). The future of ultra-high field MRI and fMRI for study of the human brain. Neurolmage, 62(2), 1241-1248.

Elul, R. (1969). Gaussian behavior of the electroencephalogram: changes during performance of mental task. Science, 164(3877), 328-331.

Emmert, K., Kopel, R., Sulzer, J., Brühl, A. B., Berman, B. D., Linden, D. E., . . Frank, S. (2016). Meta-analysis of real-time fMRI neurofeedback studies using individual participant data: How is brain regulation mediated? Neurolmage, $124,806-812$.

Everitt, B. J., Dickinson, A., \& Robbins, T. W. (2001). The neuropsychological basis of addictive behaviour. Brain Research Reviews, 36(2-3), 129-138.

Fechner, G. (1860). Elemente der Psychophysik (Elements of Psychophysics)(HE Adler, Trans.). New York: Holt, Rinehart and Winston. Inc.(1860/1966).

Fetz, E. E. (1969). Operant conditioning of cortical unit activity. Science, 163(3870), 955-958.

Fetz, E. E. (1999). Real-time control of a robotic arm by neuronal ensembles. Nature neuroscience, 2(7), 583. 
Forschner, M. (2018). Die Theorie der Affekte Die Philosophie der Stoa (pp. 224 245): Theiss.

Garrison, K., Santoyo, J., Davis, J., Thornhill, T., Kerr, C., \& Brewer, J. (2013). Effortless awareness: using real time neurofeedback to investigate correlates of posterior cingulate cortex activity in meditators' self-report. Frontiers in human neuroscience, 7, 440 .

Gatchel, R. J., Hatch, J. P., Maynard, A., Turns, R., \& Taunton-Blackwood, A. (1979). Comparison of heart rate biofeedback, false biofeedback, and systematic desensitization in reducing speech anxiety: Short-and long-term effectiveness. Journal of Consulting and Clinical Psychology, 47(3), 620.

Gazzaniga, M., Ivry, R., \& Mangun, G. (2009). A brief history of cognitive neuroscience. Cognitive Neuroscience: The Biology of the Mind. 3rd ed. New York: WW Norton \& Company Inc, 1-17.

Gerin, M. I., Fichtenholtz, H., Roy, A., Walsh, C. J., Krystal, J. H., Southwick, S., \& Hampson, M. (2016). Real-time fMRI neurofeedback with war veterans with chronic PTSD: a feasibility study. Frontiers in psychiatry, 7, 111.

Goldstein, D. S., Ross, R. S., \& Brady, J. V. (1977). Biofeedback heart rate training during exercise. Biofeedback and Self-regulation, 2(2), 107-125.

Grech, R., Cassar, T., Muscat, J., Camilleri, K. P., Fabri, S. G., Zervakis, M., . . . Vanrumste, B. (2008). Review on solving the inverse problem in EEG source analysis. Journal of neuroengineering and rehabilitation, 5(1), 25.

Greer, S. M., Trujillo, A. J., Glover, G. H., \& Knutson, B. (2014). Control of nucleus accumbens activity with neurofeedback. Neurolmage, 96, 237-244.

Gröne, M., Dyck, M., Koush, Y., Bergert, S., Mathiak, K., Alawi, E., . . Mathiak, K. (2015). Upregulation of the rostral anterior cingulate cortex can alter the perception of emotions: fMRI-based neurofeedback at 3 and 7 T. Brain topography, 28(2), 197-207. 
Gross, J., \& Thompson, R. (2007). Emotion Regulation: Conceptual Foundations. In J. Gross (Ed.), Handbook of emotion regulation (pp. 3 - 27). New York: Guilford.

Grossberg, S. (1971). On the dynamics of operant conditioning. Journal of Theoretical Biology, 33(2), 225-255.

Haller, S., Birbaumer, N., \& Veit, R. (2010). Real-time fMRI feedback training may improve chronic tinnitus. European Radiology, 20(3), 696-703.

Hanlon, C. A., Hartwell, K. J., Canterberry, M., Li, X., Owens, M., LeMatty, T., . . . George, M. S. (2013). Reduction of cue-induced craving through realtime neurofeedback in nicotine users: the role of region of interest selection and multiple visits. Psychiatry Research: Neuroimaging, 213(1), 79-81.

Hickok, G., Houde, J., \& Rong, F. (2011). Sensorimotor integration in speech processing: computational basis and neural organization. Neuron, 69(3), 407-422.

Hoellen, B. (1986). Stoizismus und rational-emotive Therapie (RET): ein Vergleich: Centaurus-Verlagsgesellschaft.

Hollon, S. D., Muñoz, R. F., Barlow, D. H., Beardslee, W. R., Bell, C. C., Bernal, G., . . Kohn, L. (2002). Psychosocial intervention development for the prevention and treatment of depression: promoting innovation and increasing access. Biological psychiatry, 52(6), 610-630.

Hwang, H.-J., Kim, K.-H., Jung, Y.-J., Kim, D.-W., Lee, Y.-H., \& Im, C.-H. (2011). An EEGbased real-time cortical functional connectivity imaging system. Medical \& biological engineering \& computing, 49(9), 985-995.

Jackson, J. (1906). The Meditations of Marcus Aurelius Antoninus: Oxford at the Clarendon Press.

Jeunet, C., Lotte, F., Batail, J.-M., Philip, P., \& Micoulaud Franchi, J.-A. (2018). Using Recent $\mathrm{BCl}$ Literature to Deepen our Understanding of Clinical 
Neurofeedback: A Short Review. Neuroscience, 378, 225-233.

Johnston, S. J., Boehm, S. G., Healy, D., Goebel, R., \& Linden, D. E. (2010). Neurofeedback: A promising tool for the self-regulation of emotion networks. Neurolmage, 49(1), 1066-1072.

Kamiya, J. (2011). The first communications about operant conditioning of the EEG. Journal of Neurotherapy, 15(1), 65-73.

Keynan, J. N., Cohen, A., Jackont, G., Green, N., Goldway, N., Davidov, A., ... Hendler, T. (2019). Electrical fingerprint of the amygdala guides neurofeedback training for stress resilience. Nature Human Behaviour, 3(1), 63-73.

Kim, D.-Y., Yoo, S.-S., Tegethoff, M., Meinlschmidt, G., \& Lee, J.-H. (2015). The inclusion of functional connectivity information into fMRI-based neurofeedback improves its efficacy in the reduction of cigarette cravings. Journal of cognitive neuroscience, 27(8), 1552-1572.

Koganemaru, S., Mikami, Y., Maezawa, H., Ikeda, S., Ikoma, K., \& Mima, T. (2018). Neurofeedback Control of the Human GABAergic System Using Noninvasive Brain Stimulation. Neuroscience, 380, 38-48.

Koob, G. F., Sanna, P. P., \& Bloom, F. E. (1998). Neuroscience of addiction. Neuron, 21(3), 467-476.

Li, X., Hartwell, K. J., Borckardt, J., Prisciandaro, J. J., Saladin, M. E., Morgan, P. S., . . . George, M. S. (2013). Volitional reduction of anterior cingulate cortex activity produces decreased cue craving in smoking cessation: a preliminary real-time fMRI study. Addiction biology, 18(4), 739-748.

Liew, S.-L., Rana, M., Cornelsen, S., Fortunato de Barros Filho, M., Birbaumer, N., Sitaram, R., . . Soekadar, S. R. (2016). Improving Motor Corticothalamic Communication After Stroke Using Real-Time fMRI Connectivity-Based Neurofeedback. Neurorehabilitation and Neural Repair, 30(7), 671-675.

Linden, D. E. J., Habes, I., Johnston, S. J., Linden, S., Tatineni, R., Subramanian, L., . . 
Goebel, R. (2012). Real-Time Self-Regulation of Emotion Networks in Patients with Depression. PloS one, 7(6), e38115.

Logothetis, N. K., Pauls, J., Augath, M., Trinath, T., \& Oeltermann, A. (2001). Neurophysiological investigation of the basis of the fMRI signal. Nature, 412(6843), 150.

Makris, N., Oscar-Berman, M., Jaffin, S. K., Hodge, S. M., Kennedy, D. N., Caviness, V. S., ... Harris, G. J. (2008). Decreased volume of the brain reward system in alcoholism. Biological psychiatry, 64(3), 192-202.

Maley, C., \& Piccinini, G. (2016). Closed Loops in Neuroscience and Compution: What It Means and Why It Matters. Paper presented at the Closed Loop Neuroscience.

Maling, N., \& McIntyre, C. (2016). Local Field Potential Analysis for Closed-Loop Neuromodulation Closed Loop Neuroscience: Elsevier Inc.

Mané, A. M., Adams, J. A., \& Donchin, E. (1989). Adaptive and part-whole training in the acquisition of a complex perceptual-motor skill. Acta Psychologica, 71(1), 179-196.

Marchesotti, S., Martuzzi, R., Schurger, A., Blefari, M. L., del Millán, J. R., Bleuler, H., \& Blanke, O. (2017). Cortical and subcortical mechanisms of brain-machine interfaces. Human Brain Mapping, 38(6), 2971-2989.

Mathiesen, C., Caesar, K., Akgören, N., \& Lauritzen, M. (1998). Modification of activity-dependent increases of cerebral blood flow by excitatory synaptic activity and spikes in rat cerebellar cortex. The Journal of physiology, 512(2), 555-566.

McDonald, A. R., Muraskin, J., Dam, N. T. V., Froehlich, C., Puccio, B., Pellman, J., . . Craddock, R. C. (2017). The real-time fMRI neurofeedback based stratification of Default Network Regulation Neuroimaging data repository. Neurolmage, 146, 157-170. 
Miezin, F. M., Maccotta, L., Ollinger, J., Petersen, S., \& Buckner, R. (2000). Characterizing the hemodynamic response: effects of presentation rate, sampling procedure, and the possibility of ordering brain activity based on relative timing. Neurolmage, 11(6), 735-759.

Modell, J. G., Mountz, J. M., Curtis, G. C., \& Greden, J. F. (1989). Neurophysiologic dysfunction in basal ganglia/limbic striatal and thalamocortical circuits as a pathogenetic mechanism of obsessive-compulsive disorder. The Journal of neuropsychiatry and clinical neurosciences.

Moritz, C. T., \& Fetz, E. E. (2011). Volitional control of single cortical neurons in a brain-machine interface. Journal of neural engineering, 8(2), 025017.

Morshed, B. I., \& Khan, A. (2014). A brief review of brain signal monitoring technologies for $\mathrm{BCl}$ applications: challenges and prospects. Journal of Bioengineering \& Biomedical Sciences, 4(1), 1.

Nagai, Y., Goldstein, L. H., Fenwick, P. B., \& Trimble, M. R. (2004). Clinical efficacy of galvanic skin response biofeedback training in reducing seizures in adult epilepsy: a preliminary randomized controlled study. Epilepsy \& Behavior, 5(2), 216-223.

Nicholson, A. A., Rabellino, D., Densmore, M., Frewen, P. A., Paret, C., Kluetsch, R., ... McKinnon, M. C. (2017). The neurobiology of emotion regulation in posttraumatic stress disorder: Amygdala downregulation via real-time fMRI neurofeedback. Human Brain Mapping, 38(1), 541-560.

Nunez, P., Silberstein, R., Cadusch, P., Wijesinghe, R., Westdorp, A., \& Srinivasan, R. (1994). A theoretical and experimental study of high resolution EEG based on surface Laplacians and cortical imaging. Electroencephalography and clinical neurophysiology, 90(1), 40-57.

Ogawa, S., Lee, T.-M., Kay, A. R., \& Tank, D. W. (1990). Brain magnetic resonance imaging with contrast dependent on blood oxygenation. Proceedings of the 
National Academy of Sciences, 87(24), 9868-9872.

Ogawa, S., Lee, T.-M., Stepnoski, R., Chen, W., Zhu, X.-H., \& Ugurbil, K. (2000). An approach to probe some neural systems interaction by functional MRI at neural time scale down to milliseconds. Proceedings of the National Academy of Sciences, 97(20), 11026-11031.

Ogawa, S., \& Lee, T. M. (1990). Magnetic resonance imaging of blood vessels at high fields: in vivo and in vitro measurements and image simulation. Magnetic resonance in medicine, 16(1), 9-18.

Paillard, J. (1996). Fast and slow feedback loops for the visual correction of spatial errors in a pointing task: a reappraisal. Canadian journal of physiology and pharmacology, 74(4), 401-417.

Peterka, R. J., \& Loughlin, P. J. (2004). Dynamic regulation of sensorimotor integration in human postural control. Journal of neurophysiology, 91(1), 410-423.

Pineda, J., Juavinett, A., \& Datko, M. (2012). Self-regulation of brain oscillations as a treatment for aberrant brain connections in children with autism. Medical hypotheses, 79(6), 790-798.

Potter, S. M., El Hady, A., \& Fetz, E. E. (2014). Closed-loop neuroscience and neuroengineering. Frontiers in neural circuits, 8, 115.

Rees, G., Friston, K., \& Koch, C. (2000). A direct quantitative relationship between the functional properties of human and macaque V5. Nature neuroscience, 3(7), 716.

Ritter, J., Gründer, K., Gabriel, G., \& Weber, M. (1971). Stoizismus Historisches Wörterbuch der Philosophie (Vol. 10, pp. 180): Schwabe Basel.

Robertson, D. (2016). The Stoic influence on modern psychotherapy. The Routledge Handbook of the Stoic Tradition. London, Routledge, 374-388.

Salles, R. (2017). The Stoic Theory of the Psychology of Action The Stoics on 
determinism and compatibilism (pp. 34-39): Routledge.

Scheinost, D., Stoica, T., Saksa, J., Papademetris, X., Constable, R., Pittenger, C., \& Hampson, M. (2013). Orbitofrontal cortex neurofeedback produces lasting changes in contamination anxiety and resting-state connectivity. Translational psychiatry, 3(4), e250.

Schultz, D. P., \& Schultz, S. E. (2008). Philosophical influences on psychology $A$ history of modern psychology (pp. 27 - 62): Cengage Learning.

Sharples, R. W. (1986). Soft determinism and freedom in early Stoicism. Phronesis, 266-279.

Shibata, K. (2012). A new neuroscientific approach using decoded neurofeedback (DecNef). Rinsho shinkeigaku= Clinical neurology, 52(11), 1185-1187.

Shibata, K., Sasaki, Y., Watanabe, T., \& Kawato, M. (2016). Response to Comment on'Perceptual Learning Incepted by Decoded fMRI Neurofeedback Without Stimulus Presentation'; How can a decoded neurofeedback method (DecNef) lead to successful reinforcement and visual perceptual learning? arXiv preprint arXiv:1612.04234.

Shin, L. M., Rauch, S. L., \& Pitman, R. K. (2006). Amygdala, medial prefrontal cortex, and hippocampal function in PTSD. Annals of the New York Academy of Sciences, 1071(1), 67-79.

Singer, T., \& Lamm, C. (2009). The social neuroscience of empathy. Annals of the New York Academy of Sciences, 1156(1), 81-96.

Sitaram, R., Ros, T., Stoeckel, L., Haller, S., Scharnowski, F., Lewis-Peacock, J., . . . Oblak, E. (2017). Closed-loop brain training: the science of neurofeedback. Nature reviews neuroscience, 18(2), 86.

Skouras, S., \& Scharnowski, F. (2018). The effects of psychiatric history and age on self-regulation of the default mode network. bioRxiv, 342220.

Sokhadze, E. M., El-Baz, A. S., Tasman, A., Sears, L. L., Wang, Y., Lamina, E. V., \& 
Casanova, M. F. (2014). Neuromodulation integrating rTMS and neurofeedback for the treatment of autism spectrum disorder: an exploratory study. Applied psychophysiology and biofeedback, 39(3-4), 237257.

Staddon, J. E. R., \& Cerutti, D. T. (2003). Operant Conditioning. Annual Review of Psychology, 54(1), 115-144.

Stein, M. B., Goldin, P. R., Sareen, J., Zorrilla, L. T. E., \& Brown, G. G. (2002). Increased amygdala activation to angry and contemptuous faces in generalized social phobia. Archives of General Psychiatry, 59(11), 1027-1034.

Stoelting, C. (1930). Apparatus, Test and Supplies for Psychology, Psychometry, Psychotechnology, Psychiatry, Neurology, Anthropology, Phonetics, Physiology, and Pharmacology. Chicago.(Collection Rand B. Evans), The Virtual Laboratory: Max-Planck-Institute for the History of Science, Berlin.

Stroman, P. W. (2016). Essentials of functional MRI: CRC Press.

Thibault, R. T., MacPherson, A., Lifshitz, M., Roth, R. R., \& Raz, A. (2017). Neurofeedback with fMRI: A critical systematic review. Neurolmage.

Toomin, M. K., \& Toomin, H. (1975). GSR biofeedback in psychotherapy: Some clinical observations. Psychotherapy: Theory, Research \& Practice, 12(1), 33.

Tourville, J. A., Reilly, K. J., \& Guenther, F. H. (2008). Neural mechanisms underlying auditory feedback control of speech. Neurolmage, 39(3), 1429-1443.

Velliste, M., Perel, S., Spalding, M. C., Whitford, A. S., \& Schwartz, A. B. (2008). Cortical control of a prosthetic arm for self-feeding. Nature, 453(7198), 1098.

Weinkauf, W. (2001a). Ethik Die Philosophie der Stoa: ausgewählte Texte (pp. 194 203): Reclam.

Weinkauf, W. (2001b). Naturphilosophie Die Philosophie der Stoa: ausgewählte Texte (pp. 105 - 113): Reclam. 
Whitehead, W. E., Drescher, V. M., Heiman, P., \& Blackwell, B. (1977). Relation of heart rate control to heartbeat perception. Biofeedback and Selfregulation, 2(4), 371-392.

Wikler, A., Norrell, H., \& Miller, D. (1972). Limbic system and opioid addiction in the rat. Experimental neurology, 34(3), 543-557.

Williamson, D. A., \& Blanchard, E. B. (1979). Heart rate and blood pressure biofeedback. Biofeedback and Self-regulation, 4(1), 1-34.

Yamashita, A., Hayasaka, S., Kawato, M., \& Imamizu, H. (2017). Connectivity Neurofeedback Training Can Differentially Change Functional Connectivity and Cognitive Performance. Cerebral Cortex, 27(10), 4960-4970.

Yoo, S.-S., \& Jolesz, F. A. (2002). Functional MRI for neurofeedback: feasibility studyon a hand motor task. Neuroreport, 13(11), 1377-1381.

Young, K. D., Misaki, M., Harmer, C. J., Victor, T., Zotev, V., Phillips, R., ... Bodurka, J. (2017). Real-time functional magnetic resonance imaging amygdala neurofeedback changes positive information processing in major depressive disorder. Biological psychiatry, 82(8), 578-586.

Young, K. D., Siegle, G. J., Zotev, V., Phillips, R., Misaki, M., Yuan, H., . . Bodurka, J. (2017). Randomized clinical trial of real-time fMRI amygdala neurofeedback for major depressive disorder: effects on symptoms and autobiographical memory recall. American Journal of Psychiatry, 174(8), 748-755.

Yuan, H., Young, K. D., Phillips, R., Zotev, V., Misaki, M., \& Bodurka, J. (2014). Restingstate functional connectivity modulation and sustained changes after realtime functional magnetic resonance imaging neurofeedback training in depression. Brain connectivity, 4(9), 690-701.

Zilverstand, A., Sorger, B., Sarkheil, P., \& Goebel, R. (2015). fMRI neurofeedback facilitates anxiety regulation in females with spider phobia. Frontiers in behavioral neuroscience, 9, 148. 
Zotev, V., Krueger, F., Phillips, R., Alvarez, R. P., Simmons, W. K., Bellgowan, P., . . Bodurka, J. (2011). Self-regulation of amygdala activation using real-time fMRI neurofeedback. PloS one, 6(9), e24522. 



\section{Chapter 2 - Two tasks are better than one: Initial motor- imagery neurofeedback can increase efficacy of rtfMRI neurofeedback-guided emotion regulation trainings}

\section{Based on:}

Skottnik, L., Sorger, B., Benjamins, C., \& Goebel, R. (in preparation). Two tasks are better than one: Initial motorimagery neurofeedback can increase efficacy of rtfMRI neurofeedback-guided emotion regulation trainings. 



\section{Abstract}

While evidence for the clinical relevance of neurofeedback-guided emotion regulation is steadily growing, a remaining draw-back of neurofeedback is its high cost intensity. Improving the effectiveness of neurofeedback interventions is therefore crucial for gaining clinical efficiency. To contribute to the efficacy of neurofeedback interventions, this proof-of-concept study investigates the effects of providing an initial neurofeedback task with assumed lower task demands before switching to the main training. Ten participants underwent three real-time fMRI neurofeedback-training sessions, in order to learn to regulate activation of the anterior insula (aINS) to three different target levels, either by performing the identical emotion regulation task over the whole training, or by first training to reach three target levels with motor-imagery-driven neurofeedback from the supplementary motor area (SMA). While achieving fine-grained control over the fMRI-signal level was more feasible for participants receiving SMA in comparison to aINS neurofeedback during the initial training, both groups were able to significantly improve their aINS regulation performance over the subsequent sessions. Although both groups did not differ in their ability to stabilize the neurofeedback signal at a certain target level, the group initially obtaining neurofeedback experience through training with a motor-imagery task showed an increased tendency to up-regulate the neurofeedback signal, which correlated with more effective training outcomes with regard to post-training positive affect. Findings were supported by participants' self-evaluation on personal neurofeedback regulation abilities and motivation. 


\section{Introduction}

Since over four decades neurofeedback has been applied in various clinical settings (first systematic clinical data presented by Sterman and Friar (1972), see Blanchard and Young (1974)), including in the treatment of major affective disorders like depression (Hammond, 2005; Linden, 2014; Mehler et al., 2018; Quaedflieg et al., 2015; Young et al., 2014), anxiety disorders (Moore, 2000; Zilverstand, Sorger, Sarkheil, \& Goebel, 2015) and addiction (Dalkner et al., 2017; Ghosh, Jahan, \& Singh, 2014; Hanlon et al., 2013; Rostami \& Dehghani-Arani, 2015). In comparison to pharmacological, surgical or brain stimulation-based intervention methods, neurofeedback trainings most commonly require active engagement from patients during the intervention. Accordingly, besides the psychological target processes used to modulate the neurofeedback signal, differences in neurofeedback training outcomes have also been related to task-general psychological factors such as motivation (Nijboer, Birbaumer, \& Kubler, 2010; Nijboer et al., 2008) and cognitive resources, like attention or memory span (Alkoby, Abu-Rmileh, Shriki, \& Todder, 2017; Daum et al., 1993; Schneider et al., 1992; Wangler et al., 2011).

While real-time functional magnetic resonance imaging (rtfMRI) neurofeedback provides the possibility to feedback brain activation from single regions or networks deep in the brain, it is tied to a novel and challenging technical environment, not present in other treatment options which also require active self-regulation (e.g., as psychotherapy or meditation). In addition to acquiring specific self-regulation skills, this demands from participants to understand the working mechanisms of neurofeedback and its relationship to the psychological target process, thereby creating a complex learning situation. 
To facilitate learning, training approaches that include different levels of task difficulty have been used since long in other learning environments (Diaz, Winsler, Atencio, \& Harbers, 1992; Porter \& Saemi, 2010; Van Zundert, Könings, Sluijsmans, \& Van Merriënboer, 2012). The current study aims to determine whether comparable training approaches can also be beneficial for neurofeedback interventions. To answer this question, we provided participants with the possibility to gain neurofeedback experience during a lesser demanding neurofeedback task, before they continued with a more advanced neurofeedback task. We expected the benefits of this approach to be twofold: On the one hand, participants could in the beginning accommodate to the environment and focus on learning the practical working mechanisms of neurofeedback, potentially allowing more cognitive resources for training the psychological target process in later training runs. On the other hand, positive neurofeedback experience acquired during a task that was subjectively experienced as being more controllable, was expected to affect task-general psychological factors as motivation and selfefficacy.

Especially in a clinical context, these general psychological factors have been shown to be of major importance: Motivation is a crucial factor for the success of psychotherapeutic interventions (for an overview see Ryan and Deci (2008)). Furthermore, the relationship between self-efficacy and positive treatment outcomes has been repeatedly shown for affective disorders as social-anxiety disorder (Goldin et al., 2012), panic disorder (Bouchard et al., 2007; Gallagher et al., 2013) and in addiction (Burleson \& Kaminer, 2005; Loeber, Croissant, Heinz, Mann, \& Flor, 2006) and is related to positive learning outcomes for interacting with novel technologies in general (Hevey, Smith, \& McGee, 1998; Mitchell \& Stuart, 1984). Finally, both motivation and self-efficacy have been shown to contribute in 
preventing drop-out from treatment interventions (Mitchell \& Stuart, 1984; Ryan, Plant, \& O'Malley, 1995).

Under these considerations, we investigated the effect of beginning a neurofeedback training with an initial task that we assumed to be less demanding for participants, compared to performing the same (more challenging) neurofeedback task over the whole training. To provide a comprehensive overview on training progression, we analysed differences between both groups with regard to neurofeedback regulation, changes in the psychological target process of the neurofeedback training and task-general psychological factors, i.e. motivation and self-efficacy. Two groups of participants underwent three real-time functional magnetic resonance (rtfMRI) neurofeedback training sessions with different training styles (Figure 1):

Participants in the first group ( $n=5)$, were asked to perform the same neurofeedback task across the whole training (constant task scenario). During all neurofeedback runs, participants in this group trained to regulate the blood-oxygen level dependent (BOLD) signal of the anterior insula (aINS) through regulation of positive emotions, aiming to stabilize its mean $\mathrm{fMRI}$ signal at three target activation levels (low, medium and high).

For participants in the second group $(n=5)$ the neurofeedback tasks changed over the course of the training (changing task scenario): During the first session, participants in this group were asked to regulate activation of the supplementary motor area (SMA) through motor imagery, aiming to stabilize its mean $\mathrm{fMRI}$ signal at low, medium and high activation levels. The SMA has been widely used as target region in neurofeedback and brain-computer interfaces studies in general (Guo, 
Zhao, Li, Yao, \& Chen, 2012; Scharnowski et al., 2004; Subramanian et al., 2016; Weiskopf et al., 2004) and it has been recently demonstrated that participants are able to gradually increase its mean BOLD activation using motor imagery to different target levels (Mehler et al., 2019; Sorger, Kamp, Weiskopf, Peters, \& Goebel, 2016). At the end of the first session, participants were provided with neurofeedback from the aINS and instructed to upregulate its BOLD-signal magnitude maximally by endogenously generating positive emotions, thereby getting familiarized with the actual psychological target process of the neurofeedback training, namely regulation of positive emotions. The subsequent two neurofeedback sessions started with neurofeedback of the SMA activation using motor imagery, but continued with modulation of the alNS using emotion regulation, in each case to three different target activation levels.

While several neurofeedback studies reported successful up- or down-regulation of the insula (Caria et al., 2007; Emmert et al., 2014; Johnston et al., 2011; Rance, Ruttorf, Nees, Schad, \& Flor, 2014), the alNS has also been implicated in neurofeedback based self-regulation in general (Emmert et al., 2016). We therefore expected it to be challenging for participants, to stabilize alNS activation around a specific target level, as aINS activation during neurofeedback training is not only driven by emotion regulation, but also fluctuates in reaction to the neurofeedback process in general. For patients, training to improve potentially dysfunctional psychological target processes can be expected to implicate a high cognitive load and potentially frustration. We therefor wanted to investigate whether a training approach with changing tasks, starting with a task that is experienced as less difficult, is especially suited, when task demands are high. 
To investigate the learning progress during neurofeedback, the current study thereby focussed on the actual visual information displayed during the neurofeedback training. While activation changes in the strengths of the fMRI signal as observed in previous studies indicate the achieved modulation of a target region by participants (Mehler et al., 2019; Sorger et al., 2016), these fluctuations are not directly perceived as feedback by participants and are therefore only indirectly linked to the subjective level of control of participants and the resulting learning process. In order to provide a comprehensive overview on how the visual feedback was regulated by participants, a twofold analysis was performed:

Firstly, we identified the ability of participants to gain fine-grained control over the neurofeedback signal, by calculating the absolute difference between the displayed activation level and the target activation level. While this performance marker indicated the total control that participants could exert over the signal, to perform fine-grained self-regulation participants had first to identify effective mental strategies that would boost the neurofeedback signal in comparison to baseline.

Secondly, we therefore identified to what extend participants increased the neurofeedback signal, and investigated how these increases were related to selfregulation performance (i.e. reaching the activation level and/or exceeding it).

By subsequently relating these two main markers of neurofeedback regulation to measures of positive affect, we could investigate in how far the different aspects of neurofeedback regulation (boosting activation and achieving fine grained control) actually related to the corresponding psychological target processes, i.e. positive affect. 
Specifically, we aimed at answering the following research objectives:

1. Do we succeed in providing participants with a less challenging neurofeedback task in the beginning of the training, i.e., do participants show superior control over the neurofeedback signal from the SMA in comparison to neurofeedback from the aINS?

2. Does a different initial neurofeedback task with lower task difficulty improve the efficacy of the neurofeedback training, i.e., does it improve the participants' ability to reach a defined target level and stabilize the signal at this level?

3. Does a different initial neurofeedback task with lower task difficulty affect the psychological target process more strongly and is this difference reflected in secondary psychological factors as motivation and self-efficacy?

\section{Methods and Design}

\subsection{Participants}

Ten healthy female participants (age: Mean $=22.9$ years, SD: 4.43 years; 1 lefthanded), all students of Maastricht University with normal or corrected-to-normal vision participated in the study. None of the participants had participated in a neurofeedback experiment before or was experienced in meditation. Participants were assigned to one of two training style groups (constant task scenario [CON] or changing task scenario [CHA]) using a minimization procedure for outcomes on relevant markers of cognitive function, MRI experience and emotional state (see 
analysis section 3.1). Before each MRI scanning session, participants gave written informed consent to participate in the study. The experimental procedure was approved by the local Ethics Committee of the Faculty of Psychology and Neuroscience at Maastricht University and participants gave their written consent to participate in the study.

\subsection{Experimental procedure}

All participants took part in a two-hour psychometric test session before the start of the neurofeedback training. Based on results of this behavioural session, participants were assigned to one of the two training-style groups (Figure 1). After acquisition of anatomical MRI and localization procedure (details below), the CHA group started the first session with SMA neurofeedback to achieve three target levels (low [20\% of the individual maxPSC], medium [50\% of the individual maxPSC] or high [ $80 \%$ of the individual maxPSC]) using motor imagery and finished the first session with a single run of neurofeedback guided up-regulation of the aINS (Figure $1 \mathrm{~A})$. In the subsequent two sessions, the CHA group started with a single run of motor-imagery-guided SMA neurofeedback aimed at reaching the target levels and continued with neurofeedback-regulation to target levels using positive emotion regulation (Figure $1 \mathrm{~B}$ ). After undergoing the same acquisition procedure of anatomical MRI and localization procedure, the CON group performed three sessions of aINS neurofeedback-regulation to target levels using positive emotion regulation across all neurofeedback runs (Figure $1 \mathrm{~A}, \mathrm{~B}$ ). All neurofeedback sessions of a participant were scheduled in subsequent calendar weeks. 


\section{Training Style}

\section{Constant \\ Changing}

A

Neurofeedback Runs

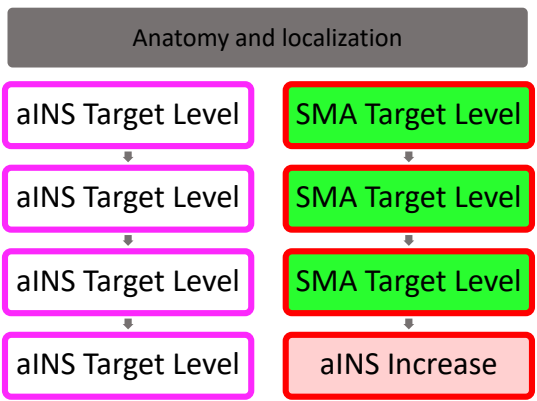

B
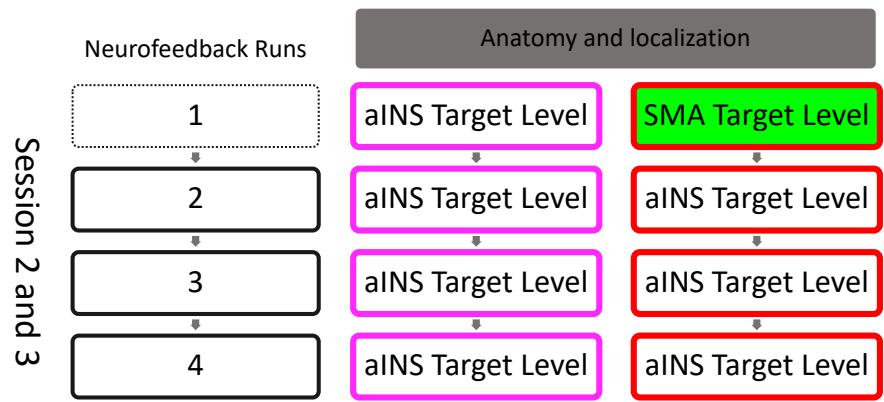

Figure 1: Study design. A. Neurofeedback training focus across sessions: The constant task group (magenta outline) performed the same emotion regulation task across all neurofeedback runs, while for the changing task group (red outline) the neurofeedback training focus varied across sessions, changing from motor imagery (receiving SMA neurofeedback, green) to emotion regulation (alNS neurofeedback). A. Training differences in the first sessions: During the first three runs, both groups regulated the neurofeedback signal to three different target levels, but differed with regard to the neurofeedback modality. The constant task group received aINS neurofeedback, while the changing task group at first received SMA neurofeedback (green), switching in the last run to upregulation of the aINS. B. Training differences in the second and third sessions: Both groups aimed at regulating the provided neurofeedback signal to three different target levels and differed only with regard to the neurofeedback modality in the first run. Runs outlined in black were used for comparing neurofeedback performance. 


\subsection{Visual stimulation and instructions}

\subsubsection{Localization procedures}

aINS localizer. Pictures with positive affect and high arousal (mean normative ratings for female participants in valence 8.23 [SD 0.23], arousal mean $=4.78$ [SD 0.74]) and neutral valence and average arousal (valence mean $=4.95$ [SD 0.07], arousal 3.17 [SD 0.68]) from the International Affective Pictures System (IAPS, Lang, Bradley, and Cuthbert (1997)) were presented in randomized fashion in blocks of four pictures ( $1.5 \mathrm{~s}$ per picture, 6 sec per picture block, 12 picture blocks per valence category), alternating with $12 \mathrm{~s}$ fixation periods. The first fixation period had a duration of $16 \mathrm{~s}$ (resulting in $448 \mathrm{~s}$ in total). Participants were instructed to observe the pictures and emerging emotions. This localization paradigm has previously been shown to localize regions involved in positive affect that can be up-regulated via generation of positive emotions guided by neurofeedback and the right anterior insula was most commonly activated across participants (Johnston et al., 2011).

SMA localizer. To determine the SMA involved in motor imagery, participants were presented with a white fixation cross during rest, which turned red when participants were asked to imagine performing a movement as vivid as possible. Rest blocks (16 s) alternated with motor-imagery blocks (20 s) until participants had performed twelve motor-imagery blocks. The first rest period had a duration of 20 $\mathrm{s}$ (resulting in $452 \mathrm{~s}$ in total).

During neurofeedback runs, rest blocks (20 s) alternated with neurofeedback blocks (30 s) until participants had performed nine neurofeedback blocks, the first rest block had a duration of 90 seconds to accommodate participants to the scanning situation (resulting in $540 \mathrm{~s}$ in total). During the whole neurofeedback run, a 
thermometer-like display was displayed (Figure 2), consisting of ten equally sized segments. During rest periods, only the thermometer (white on black background) was visible and participants were instructed not to think of anything particular. During neurofeedback trials, a certain segment of the thermometer was marked red and participants were instructed to increase the appearing neurofeedback signal (depicted by segments filled in grey) and stabilize it at the height of the target level. Apart from the last run of the first session for the CHA group (where only the highest segment [10] was marked red), the selected segments were 2, 5 and 8, alternating in random order.

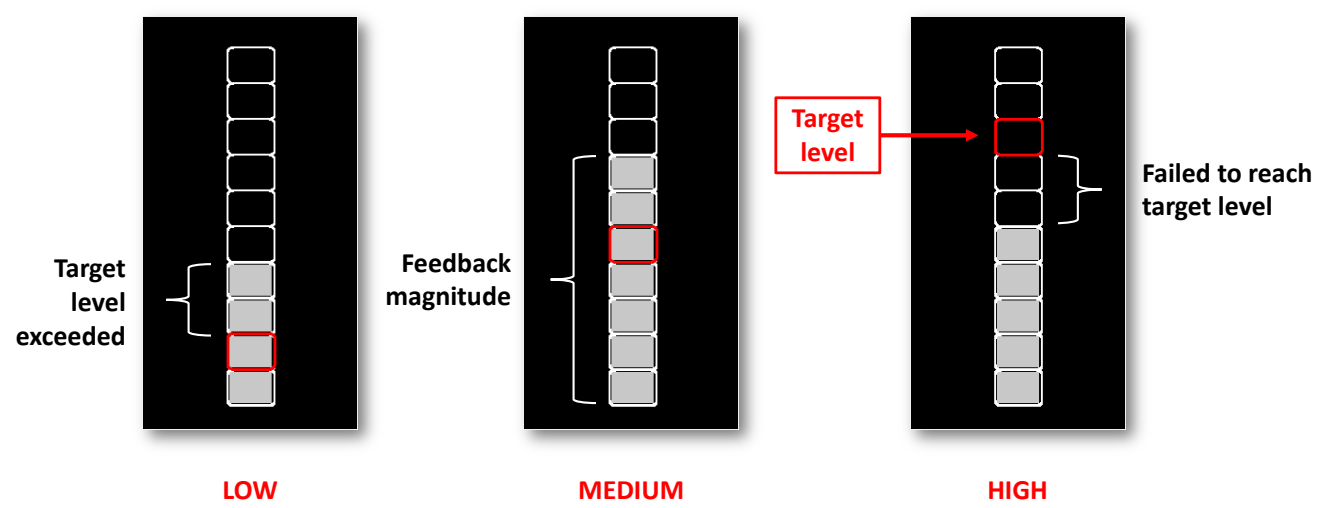

Figure 2: Neurofeedback display and markers of performance

Neurofeedback was provided by filling rectangular segments of a vertical thermometer grey, according to the current BOLD-signal magnitude in a participant's neurofeedback target region. During active task conditions, one segment of the thermometer was marked red, indicating the target activation level. During target-level runs, segments 2, 5 and 8 were marked interchangeably in a block-like fashion. Participants trained to increase the neurofeedback signal until they reached the target level and then stabilize the signal. They could deviate from the task goal by failing to reach the target level or by exceeding it. 


\subsubsection{Self-regulation instructions}

In the psychometric session, participants in both groups were informed that they would train to achieve control over brain activation within a region of their brain involved in emotion regulation, and were briefly introduced to the target-level design. Instructions for both groups differed only with regard to task instructions during the actual neurofeedback sessions, where the CHA group was additionally provided with instructions for SMA self-regulation through motor imagery and neurofeedback.

For regulating the aINS neurofeedback signal, participants were provided with the information, that the neurofeedback signal originated from a brain region involved in positive emotions. It was suggested to participants to increase the signal by imagining or remembering positive events but that they were free to explore and identify their personal strategy, which was most effective to reach the different target levels. For regulating the SMA-neurofeedback signal, participants were provided with the information that the neurofeedback signal originated from a brain region involved in initiating movements. It was suggested to participants to increase the signal by imagining a movement while focusing on the somaticexperience accompanied by this movement, but that they were free to explore and identify their personal strategy, which was most effective to reach the target level. To down-regulate the neurofeedback signal across both types of training, participants were instructed to vary the intensity of their mental operation or to focus on something task-irrelevant. 


\subsection{Data acquisition}

\subsubsection{Psychometric measures and questionnaires acquired during the pre-testing session}

During the psychometric pre-testing session, positive trait affect was acquired using the Positive Affect Negative Affect Scale Trait (PANAS Trait), positive scale (Watson, Clark, \& Tellegen, 1988), for motivation to participate in the training the questionnaire of current motivation, QCM (Freund, Kuhn, \& Holling, 2011). Fullscale IQ was estimated using the matrix reasoning and the vocabulary subscale of the Wechsler Adult Intelligence Scale (WAIS-IV).

\subsubsection{Post-scanning questionnaires}

At the end of each neurofeedback session, the affective state of participants was acquired using the PANAS State (Watson et al., 1988), motivation during the experiment using the QCM (Freund et al., 2011) and an estimate of neurofeedback skills and self-efficacy using the Neurofeedback Skills Questionnaire (NSQ). The NSQ is a questionnaire developed to measure self-efficacy to control a provided neurofeedback signal, as well as the extend of explicit knowledge on how to control the signal (see Supplementary Material SI). The NSQ consists of two parts: First, a qualitative part based on semi-structured questions assesses the content of selfregulation strategies as well as the efficacy of the strategies to regulate the neurofeedback signal. Second, a quantitative part based on Likert scales to assesses self-efficacy and knowledge of effective self-regulation strategies. 


\subsection{3 (F)MRI data}

All MR images were recorded using a 3-T Siemens Magnetom Prisma MRI system (Siemens, Erlangen, Germany) with a 64-channel receiver head coil.

Structural Data acquisition

All participants underwent a T1-weighted anatomical scan using a threedimensional (3D) magnetization prepared rapid-acquisition gradient-echo (MPRAGE) sequence (192 slices, slice thickness $=1 \mathrm{~mm}$, no gap, $\mathrm{TR}=2250 \mathrm{~ms}, \mathrm{TE}=$ $2.21 \mathrm{~ms}, \mathrm{FA}=9, \mathrm{FOV}=256 \times 256 \mathrm{~mm}^{2}$, matrix size $=256 \times 256$ ) in every MRI session.

\section{Functional Data acquisition}

Functional images were acquired with a $\mathrm{T} 2 *$-weighted echo-planar imaging (EPI) sequence. Except for the number of acquisitions (aINS localizer: 448 volumes; SMA localizer: 452 volumes; neurofeedback runs: 540 volumes), identical scanning parameters were used for all functional measurements (48 slices, slice thickness $=$ $2 \mathrm{~mm}, 1 \mathrm{~mm}$ gap, $\mathrm{TR}=1000 \mathrm{~ms}, \mathrm{TE}=30 \mathrm{~ms}, \mathrm{FA}=50, \mathrm{FOV}=210 \times 210 \mathrm{~mm}^{2}$, matrix size $=70 \times 70$, multi-band acceleration factor $=3$, slice encoding direction $=$ anterior to posterior).

\section{Analysis}

\subsection{Matching}

Participants were assigned to one of two training style groups using a minimization procedure (sequential balancing, Borm, Hoogendoorn, den Heijer, and Zielhuis (2005)) with the factors previous participation in MRI experiments, estimated IQ (weighted sum of vocabulary and matrix reasoning of WAIS-IV, Pearson Education, 
Inc., San Antonio, TX), positive trait affect (PANAS-Trait, Watson et al. (1988), positive scale), motivation to participate in the training (Freund et al., 2011).

\subsection{Neurofeedback preparation and generation}

\subsubsection{Analysis of anatomical data}

Analysis of anatomical data was performed in Brainvoyager QX Version 2.8 Anatomical data sets were corrected for spatial intensity inhomogeneity. For all participants, the anatomical data set from the first session was transferred into ACPC space and the anatomical data set from the second and third session was automatically aligned to the ACPC version of the first data set. For post-hoc analysis, anatomical data-sets were accordingly transferred into Talairach space (Talairach \& Tournoux, 1988).

\subsubsection{Creation of neurofeedback target regions}

Neurofeedback target regions were defined in Turbo-Brain Voyager (V2.8, Brain Innovation B.V., Maastricht, the Netherlands) based on activation during the localizer runs: During the first neurofeedback session, a general linear model (GLM) was calculated on the pre-processed (motion corrected, linear trend removed and aligned to anatomical scans in ACPS space) functional data obtained during the localizer runs. From the corresponding contrast (positive pictures vs. rest [aINS localizer] and motor imagery vs. rest [SMA localizer], functional voxels were selected based on the maximal F-values located in the anatomically defined areas in which the specific regions of interest were defined (aINS for emotion regulation and SMA for motor imagery, Table 1, Figure 3). Around the peak voxel of an 
activation cluster all touching voxels in the same functional slice were selected $(3 * 3$ voxel in one slice), as well as the corresponding voxels in the slices below and above the central-voxel (resulting in a square shaped ROI: $3 * 3 * 3$ voxel in total). The central voxel of the square was interpolated to $1 * 1 * 1$ voxel and saved in ACPC space (this was done in order to guide ROI selection during subsequent sessions, see Supplementary Figure SII)).

In the second and third session, a short resting-state localizer scan was performed (10 volumes) to determine the position of functional slices. Functional data was aligned to the T1-weighted images of the respective session, which was aligned to the anatomical images of session one in ACPC space. Subsequently, the region of interest was created by selecting $3 * 3 * 3$ voxel in direct proximity to the central voxel of the first session's ROI, that corresponded to the respective anatomically defined areas of each neurofeedback condition (SMA or aINS).

This approach supported the selection of corresponding functional voxels despite slight differences in slice positioning across sessions. Furthermore, this allowed to create regions of interest of the same size in 2D functional space, despite several interpolations between 2D and 3D space with differing voxel sizes (3- $\mathrm{mm}$ isovoxel for functional data, $1 \mathrm{~mm}$ isovoxel for T1-weighted scans), slight differences in sizes remained due to differences in slice orientation and manual selection errors (see Tables 1 and 2). For further offline-analysis, the 2D functional ROls where interpolated to 3D volumes of interest (VOIs) and transferred into ACPC space. 


\subsubsection{Generation of neurofeedback information}

The neurofeedback signal was calculated by following the general procedure previously validated for neurofeedback target-level designs by Sorger et al. (2016): In short, the percent signal change (PSC) of the current pre-processed volume of the consecutive runs was calculated as mean activation of all voxels within the particular ROI, with respect to a local baseline. For the current study, the percent signal change (PSC) of a given volume was calculated as the temporarily smoothed (by a factor of 6; i.e., the mean of the current and the five previous time-points) to stabilize the feedback signal acquired with the relatively high TR of $1000 \mathrm{~ms}$. The average activation during the preceding rest block constituted the baseline for the consecutive neurofeedback block. The PSC was then normalized by a maximum PSC (maxPSC), which was defined as follows: For emotion regulation, the maxPSC was set to $1 \%$ for all participants, to verify comparability between groups. For SMA regulation, the maxPSC was determined based on the mean percent signal change in the selected voxels of the localizer run (mean $=1.4 \%, S D=0.42 \%$; Table 1,2 ). The PSC at each TR was transformed into a feedback value on a scale between 0 and 10 , where 10 corresponded to maxPSC and each segment to $10 \%$ of the maxPSC in relation to the previous baseline period, so that the target levels $3,5,8$ corresponded to $30 \%, 50 \%$ and $80 \%$ of the maxPSC. In the thermometer display provided to participants, values below zero and above 10 were displayed as " 0 " and "10", respectively.

\subsection{Post-hoc analysis of neurofeedback data}

Analysis of neurofeedback performance was performed in MATLAB (v8.1 R13; The MathWorks, Natick, USA), besides analysis of variance (ANOVA), which was performed in SPSS (IBM Corp. Released 2013. SPSS, Version 22.0. Armonk, NY: IBM 
Corp). Values of feedback magnitude were extracted from the neurofeedback stimulation images (as presented to the participant during the neurofeedback experiment), resulting in one value of neurofeedback magnitude per scanned volume during modulation periods. For each scanned volume during modulation periods, one index of self-regulation accuracy was created by calculating the absolute difference between the target level and the feedback magnitude actually achieved by the participant (Figure 2).

\subsubsection{Comparing regulation performance between SMA and aINS neurofeedback (data of session 1)}

The difference in absolute distance to target level (i.e. the absolute difference between the achieved activation and the respective target level) between groups was tested using an independent samples t-test on the participants' mean absolute distance to target level for SMA neurofeedback and aINS neurofeedback (Figure 3). To determine the relationship between feedback magnitude and desired target level, a linear trend line was fitted between the target level values (20\% 50\%, 80\%) and the actual mean PSC achieved using Pearson's r. Gradual up-regulation performance of individual participants was investigated by obtaining Pearson's $r$ between single- trial means of feedback magnitude and the corresponding target levels. Obtained correlation coefficients were subsequently Fishers z-transformed for comparability. To examine potential interactions between gradual upregulation of the feedback signal and the neurofeedback modality provided in the initial session of each training style (CON: Emotion regulation, alNS feedback; DIF = Motor imagery, SMA feedback) on the population level, a two way between subjects ANOVA was calculated on the mean feedback magnitude for the factors FEEDBACK CONDITION (SMA, aINS) and TARGET LEVEL (low, medium, high). 


\begin{tabular}{|c|c|c|c|c|c|c|}
\hline \multicolumn{2}{|c|}{ Participants } & \multicolumn{3}{|c|}{ Center of mass } & \multicolumn{2}{|l|}{ Size } \\
\hline $\mathrm{CON}$ & Region & $x$ & $y$ & $z$ & Voxel & MaxPSC \\
\hline P01 & aINS & 43.02 & 8.75 & 2.32 & 724 & 1 \\
\hline P03 & aINS & 40.62 & 17.23 & 8.08 & 899 & 1 \\
\hline P05 & aINS & 45.62 & 19.54 & 3.06 & 745 & 1 \\
\hline P06 & aINS & 45.7 & 12.83 & 3.66 & 780 & 1 \\
\hline P10 & aINS & 35.45 & 17.31 & 9.44 & 838 & 1 \\
\hline \multicolumn{7}{|l|}{ CHA } \\
\hline $\mathrm{P} 02$ & SMA & -0.01 & -4.58 & 57 & 590 & 1 \\
\hline P04 & SMA & -1.93 & -11.29 & 55 & 585 & 2 \\
\hline P07 & SMA & -2.46 & -11.99 & 63.76 & 721 & 1.5 \\
\hline P08 & SMA & -3.05 & -12.64 & 63.69 & 642 & 1 \\
\hline P09 & SMA & -0.44 & -8.53 & 67.02 & 673 & 1.5 \\
\hline
\end{tabular}

Table 1: Properties of the neurofeedback target regions of session one. Position of individual participants' neurofeedback target regions during the first session in Talairach space, corresponding maxPSC values used for neurofeedback generation. Coordinates are in Talairach space.

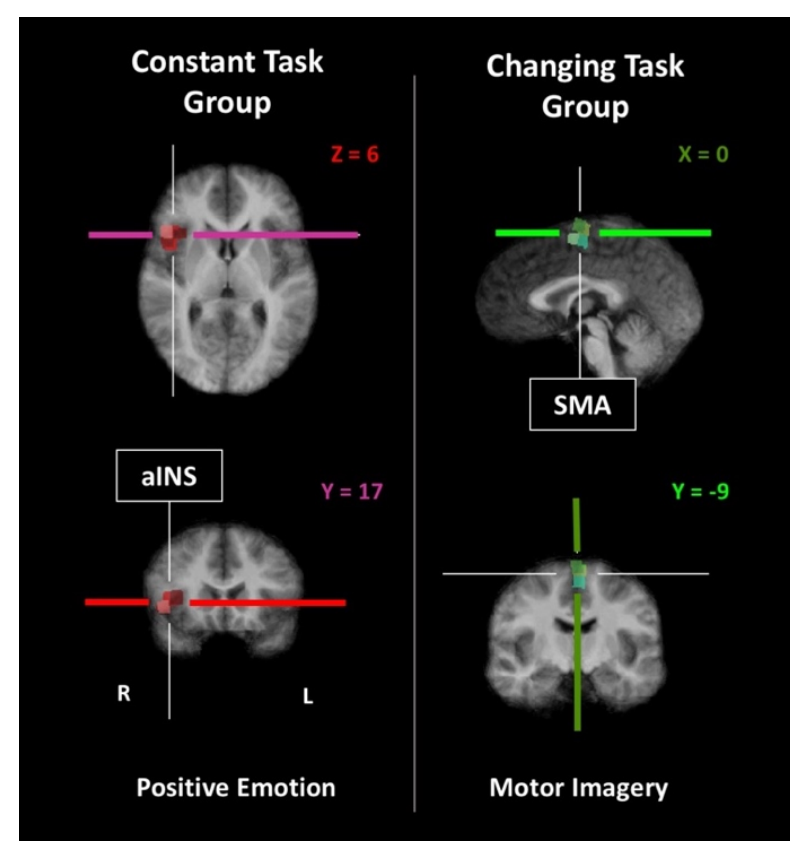

Figure 3: 3D reconstructions of neurofeedback target regions used for SMA and aINS performance comparison A. Volume representations of neurofeedback target regions during the first neurofeedback session for the constant task group (left side, aINS, magenta) and the changing task group (right side, SMA, green), shadings indicate individual participants. Regions are overlaid on the average group anatomy. 


\subsubsection{Comparing regulation performance between training styles}

\section{i. Consistency of aINS ROIs between groups}

Before comparing group performances during alNS neurofeedback, consistency of aINS ROIs across all sessions was assessed by interpolating the 2D functional ROIs to $3 \mathrm{D}$ volumes of interest (VOIs) and transferring them into ACPC and Talairach space (Table 2, Figure 4). Subsequently, the overlap of voxels between sessions was assessed within participants (logical AND). Separately for all combinations of sessions, the percentage of all voxels that overlapped from in a given comparison was calculated within participants, as for example: (overlapping voxel [session $2 \&$ $3]$ / total amount of voxel [session $2+3]) * 100$. Finally, consistency across sessions was compared between groups using independent samples t-test.

ii. Comparing aINS regulation performance between training styles (session 2 \& 3) To examine the effect of training and time on fine grained control over the feedback signal from the alNS (Table 2), a two-way between subjects ANOVA was performed on the mean absolute distance to target level with the factors TRAINING STYLE (constant task, changing task) * TIME (Session 2, 3). To examine the effect of training and time on fine-grained up-regulation of the feedback signal, a three-way between subjects ANOVA was performed on the mean feedback magnitude for the factors TRAINING STYLE (constant task, changing task) * TIME (Session 2, 3) * TARGET LEVEL (low, medium or high). Gradual up-regulation performance of individual participants was investigated by obtaining Pearson's $r$ between singletrial means of feedback magnitude and the corresponding target levels during the last session. Obtained correlation coefficients were subsequently Fishers ztransformed for comparability. 


\begin{tabular}{|c|c|c|c|c|c|}
\hline \multirow[b]{2}{*}{ Participant } & \multirow[b]{2}{*}{ Session } & \multicolumn{3}{|c|}{ Center of mass } & \multirow{2}{*}{$\begin{array}{c}\text { Size } \\
\text { Voxel }\end{array}$} \\
\hline & & $x$ & $y$ & $z$ & \\
\hline \multirow{3}{*}{ P01 } & 1 & 43.02 & 8.75 & 2.32 & 724 \\
\hline & 2 & 46.80 & 8.35 & 3.51 & 778 \\
\hline & 3 & 44.03 & 6.68 & 2.91 & 752 \\
\hline \multirow{3}{*}{$\underline{\mathbf{P 0 2}}$} & $\mathbf{1}$ & 38.12 & 24.51 & -0.93 & 675 \\
\hline & 2 & 36.83 & 22.83 & -1.28 & 656 \\
\hline & 3 & 36.89 & 22.46 & 3.06 & 644 \\
\hline \multirow{3}{*}{ P03 } & $\mathbf{1}$ & 40.62 & 17.23 & 8.08 & 899 \\
\hline & 2 & 40.88 & 17.87 & 7.56 & 850 \\
\hline & 3 & 40.96 & 14.42 & 9.48 & 820 \\
\hline \multirow{3}{*}{$\underline{\text { P04 }}$} & $\mathbf{1}$ & 43.97 & 12.28 & 4.76 & 828 \\
\hline & 2 & 41.07 & 11.62 & 5.09 & 869 \\
\hline & 3 & 39.83 & 9.58 & 3.20 & 796 \\
\hline \multirow{3}{*}{ P05 } & $\mathbf{1}$ & 45.62 & 19.54 & 3.06 & 745 \\
\hline & 2 & 40.88 & 17.87 & 7.56 & 850 \\
\hline & 3 & 40.15 & 8.82 & 2.51 & 851 \\
\hline \multirow{3}{*}{ P06 } & $\mathbf{1}$ & 45.70 & 12.83 & 3.66 & 780 \\
\hline & 2 & 44.88 & 11.25 & 4.71 & 762 \\
\hline & 3 & 41.09 & 13.01 & 3.61 & 754 \\
\hline \multirow{3}{*}{$\underline{\mathbf{P 0 7}}$} & 1 & 39.72 & 18.12 & 3.30 & 852 \\
\hline & 2 & 38.55 & 20.54 & 8.67 & 870 \\
\hline & 3 & 38.17 & 15.43 & 2.92 & 872 \\
\hline \multirow{3}{*}{$\underline{\mathbf{P 0 8}}$} & 1 & 34.00 & 21.38 & 6.35 & 839 \\
\hline & 2 & 40.30 & 19.99 & 2.79 & 879 \\
\hline & 3 & 35.32 & 21.38 & 8.61 & 855 \\
\hline \multirow{3}{*}{$\underline{\text { P09 }}$} & $\mathbf{1}$ & 38.74 & 22.48 & 3.04 & 730 \\
\hline & 2 & 37.20 & 20.88 & 5.19 & 757 \\
\hline & 3 & 33.84 & 20.13 & 4.81 & 767 \\
\hline \multirow{3}{*}{ P10 } & 1 & 35.45 & 17.31 & 9.44 & 838 \\
\hline & 2 & 37.00 & 14.53 & 8.03 & 776 \\
\hline & 3 & 36.15 & 15.62 & 7.42 & 786 \\
\hline
\end{tabular}

Table 2: Neurofeedback target regions for the affective core training. Three-dimensional volume representations of neurofeedback target-regions of each participant and across groups for the affective core training. The table contains the positions of all alNS regions in Talairach space and respective size in voxel, all maxPSCs $=1 \%$. Non-underlined participants: CON group; Underlined participants = CHA group. Coordinates are in Talairach space . 

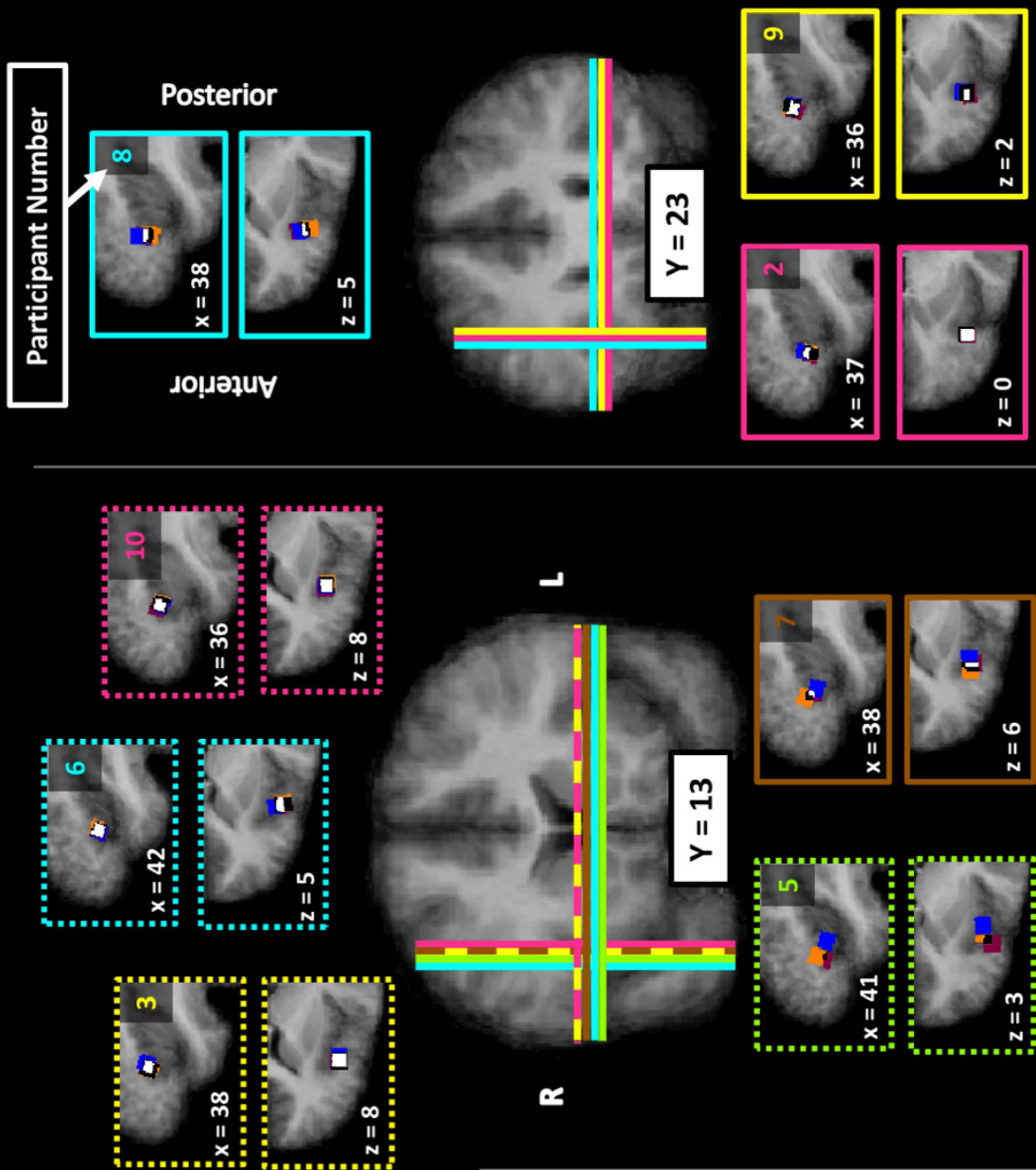

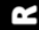
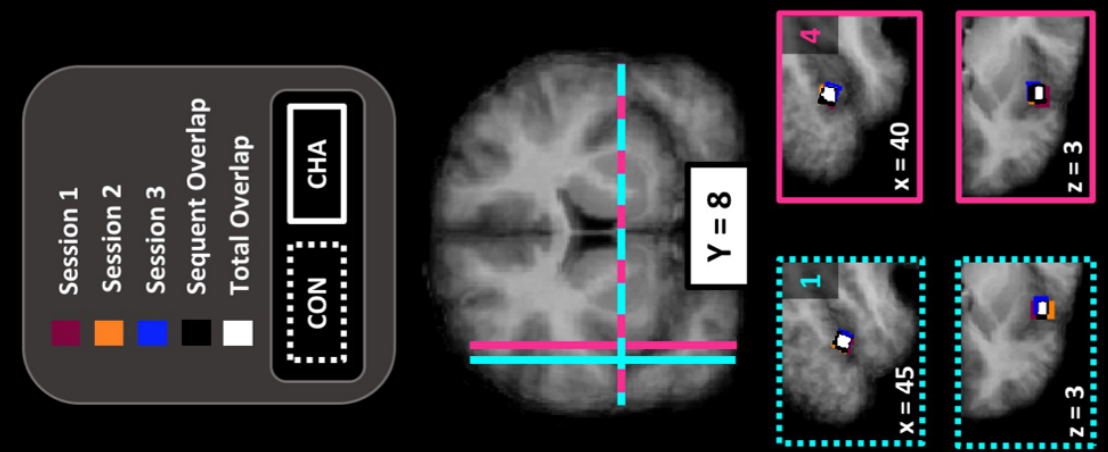
Figure 4: 3D reconstructions of aINS neurofeedback target regions. aINS target regions of single participants across time (session 1 = bordeaux, 2 = orange, 3 = blue; see Table 2 ). Colored numbers represent participant numbers. Outline type of single participants indicate group (dashed: constant task; solid: changing task). Within participants, all target ROls overlapped with target ROIs of the following training session (i.e. session 1 with 2 or session 2 with 3; black), and for nine of ten participants target regions overlapped across all sessions (white), see Table 4. Regions are overlaid on the average group anatomy in Talairach space.

\subsubsection{Psychological outcomes measures}

i. Relationship between alNS neurofeedback performance and positive affect

PANAS scores for positive affect were determined as described in Watson et al. (1988). To examine whether the mental operations used by participants to modulate the neurofeedback signal were reflective of the psychological target processes (positive affect), we investigated whether the magnitude of the neurofeedback signal during the last session (where participants were at the maximum of their neurofeedback-regulation abilities) could predict positive affect directly after the training by calculating the correlation between the mean magnitude of the neurofeedback signal during the last session, to PANAS scores after the training. Furthermore, to examine whether the effectiveness of the training differed between groups, we investigated whether neurofeedback performance during the last session could predict positive affect differentially between both groups. For this reason, the Pearson's correlation coefficients between the mean absolute distance to target level and the PANAS positive score was calculated within groups and compared according to Eid, Gollwitzer, and Schmitt (2010). 


\section{ii. Secondary psychological outcome measures}

QCM scores were determined as described in Freund et al. (2011).

Scores for the quantitative sub-scale of the NSQ were determined as described in Supplementary Material SI. Categories for answers on the qualitative sub-scale of the NSQ were created as follows: Mental processes driving the neurofeedback signal in the aINS were categorized according to a recent meta-analysis on anterior insula function ( $n=811$; Kurth, Zilles, Fox, Laird, and Eickhoff (2010)), that reported insula activation across the categories "emotion", "empathy", "olfaction", "gustation", "interoception, "pain", "somatosensation", "motion", "attention", "language", "speech, "working memory", "memory". For grouping regulation strategies applied to increase the aINS neurofeedback signal, general cognitive categories were excluded ("attention", "working memory", "memory", "language" / "speech"), as these processes were implicated in most strategies applied by participants. Additionally, the meta-analysis revealed no specific activation peaks for "attention", "language" and "motion" in the insula, indicating that these categories highly covary with other categories. "Motion" was therefor also omitted and "pain" due to its antagonistic relationship with positive affect. Strategies described by participants were therefore allocated to belong to one of the following categories: Focus of attention on positive affect, empathy, olfaction, gustation, interoception. For each session, it was noted how many different mental categories participants explored and how effective the categories appeared to be for participants. 


\section{Results}

\subsection{Matching}

Both groups were matched with regard to estimated IQ (weighted sum of vocabulary and matrix reasoning of WAIS-IV, Pearson Education, Inc., San Antonio, TX), positive trait affect (Positive and Negative Affect Schedule Trait version (PANAS-Trait), Watson et al. (1988), positive scale), motivation to participate in the training (Freund et al., 2011) and did not differ significantly on any of the variables, Table 3.

\begin{tabular}{|c|c|c|c|c|c|c|c|}
\hline CON & $I Q$ & PANAS & $Q C M$ & Age & & Female & MRI experience \\
\hline Mean & 106.2 & 35.2 & 4.63 & 21.80 & Sum & 5 & 3 \\
\hline$S D$ & 7.4 & 7.56 & 0.7 & 2.39 & - & - & - \\
\hline \multicolumn{8}{|l|}{ CHA } \\
\hline Mean & 103.2 & 38.6 & 4.77 & 24.20 & Sum & 5 & 3 \\
\hline$S D$ & 10.9 & 3.05 & 0.45 & 5.8 & - & - & - \\
\hline p-value & 0.62 & 0.38 & 0.72 & 0.42 & Difference & 0 & 0 \\
\hline t-value & 0.51 & -0.93 & -0.37 & -0.86 & - & - & - \\
\hline
\end{tabular}

Table 3: Matching variables. Descriptive statistics for matching variables between groups and results for between groups difference tests. Groups were matched on IQ, positive trait affect (PANAS-Trait positive), motivation to participate in the training (QCM) and MRI experience and gender (all participants were female).

\subsection{Differences between SMA and aINS neurofeedback}

In the first session, regulation of neurofeedback from the aINS (by means of positive emotion regulation) was associated with larger absolute distance between the target activation level and the actually achieved magnitude of the neurofeedback, in comparison to regulation of SMA neurofeedback (by means of motor imagery), although the difference was not significant (absolute distance: aINS mean $=4.24$, $\mathrm{SE}=0.11 ; \mathrm{SMA}$ mean $=3.92, \mathrm{SE}=0.18 ; p=0.16$, Cohen's $d=1.0$; Figure $5 \mathrm{~A}$ ). On the 
level of single participants, results were supportive for group findings: All participants in the SMA group showed lower mean absolute distance to the target level than $80 \%$ participants receiving alNS feedback (Table 4).

The alNS neurofeedback group did not achieve an overall gradual increase with target level in the first session (feedback magnitude: low target level mean $=2.45$, $\mathrm{SE}=0.62$; medium target level mean $=2.81, \mathrm{SE}=0.50$; high target level mean $=$ 2.42, SE $=0.33 ; r=-0.07, p=0.96$, Figure $5 \mathrm{~B}$ ). The SMA neurofeedback group significantly increased the feedback magnitude accordingly to the relative height of the target level (feedback magnitude: low target level mean $=2.97, \mathrm{SE}=0.71$; medium target level mean $=3.61, \mathrm{SE}=0.40$; high target level mean $=4.10, \mathrm{SE}=$ $0.72 ; r=0.997, p=0.05$. On average, magnitude of the neurofeedback signal was higher for the SMA neurofeedback group (feedback magnitude: aINS mean $=2.57$ $\mathrm{SE}=0.44 ; \mathrm{SMA}$ mean $=3.57, \mathrm{SE}=0.43$ ) but the ANOVA revealed no significant differences on population level (main effect FEEDBACK MODALITY: $F(1,8)=2.61, p$ $=0.15$, main effect TARGET LEVEL: $F(2,16)=0.94, p=0.41$; Interaction FEEDBACK MODALITY * TARGET LEVEL: $F(1,8)=0.93, p=0.42$. 


\begin{tabular}{|c|c|c|c|c|c|}
\hline \multicolumn{2}{|c|}{ Participants } & \multirow{2}{*}{$\begin{array}{l}\text { Absolute } \\
\text { Mean }\end{array}$} & \multirow{2}{*}{$\begin{array}{r}\text { Distance } \\
\text { SE }\end{array}$} & \multirow{2}{*}{$\begin{array}{c}\text { Single-Trial } \\
R(z)\end{array}$} & \multirow{2}{*}{$\begin{array}{c}\text { Correlation } \\
p \\
\end{array}$} \\
\hline CON & Region & & & & \\
\hline P01 & aINS & 4.38 & 0.20 & -0.16 & 0.45 \\
\hline P03 & aINS & 4.27 & 0.29 & 0.04 & 0.86 \\
\hline P05 & aINS & 4.48 & 0.12 & -0.34 & 0.10 \\
\hline P06 & aINS & 3.85 & 0.28 & 0.29 & 0.15 \\
\hline $\mathrm{P} 10$ & aINS & 4.21 & 0.34 & 0.15 & 0.46 \\
\hline Mean & & 4.24 & 0.24 & 0.00 & - \\
\hline \multicolumn{6}{|l|}{ CHA } \\
\hline $\mathrm{P} 02$ & SMA & 4.17 & 0.07 & 0.10 & 0.64 \\
\hline P04 & SMA & 3.94 & 0.44 & 0.12 & 0.56 \\
\hline P07 & SMA & 4.09 & 0.09 & 0.17 & 0.39 \\
\hline P08 & SMA & 3.22 & 0.05 & 0.80 & 0.00 \\
\hline P09 & SMA & 4.16 & 0.28 & -0.04 & 0.83 \\
\hline Mean & & 3.92 & 0.18 & 0.23 & - \\
\hline
\end{tabular}

Table 4: Task performance of individual participants during session one

Task performance with regard to achieved mean absolute distance to target-level and gradual up-regulation ability on single-trial level (correlation between neurofeedback magnitude and target levels).

Consistency within groups regarding gradual up-regulation ability was further investigated by analysing gradual-upregulation ability on the level of single participants (correlation of mean single-trial neurofeedback magnitude with target levels): Nearly all participants in the SMA group (4/5) but only two participants in the alNS group achieved a positive trend correlation $(r>0.01)$ with target levels $(\mathrm{CON}$ mean $\mathrm{r}(\mathrm{z}$-value $)=-0.004 ; \mathrm{SE}=0.11 ; \mathrm{CHA}$ mean $\mathrm{r}(\mathrm{z}$-value $)=0.23 ; \mathrm{SE}=0.15$; Table 4). 
A

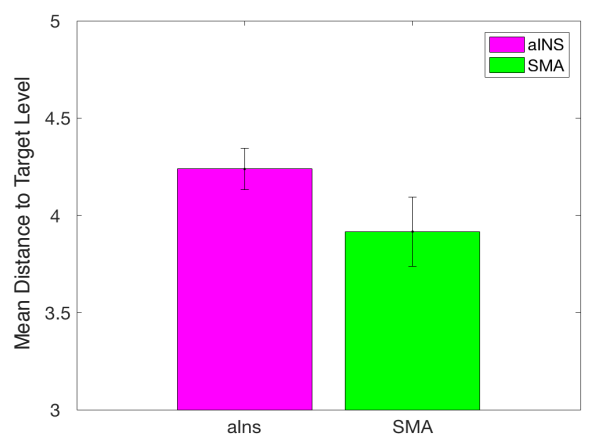

B

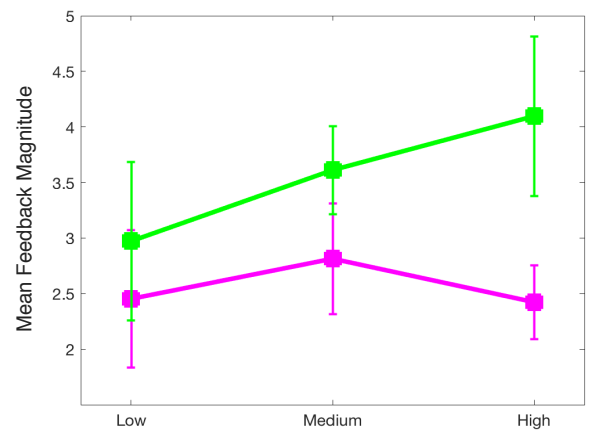

Participant

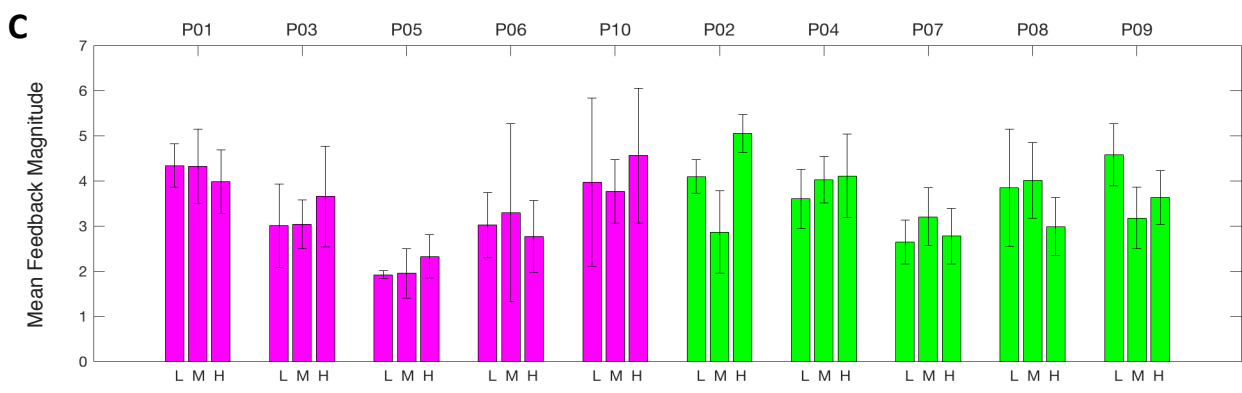

Target Level

Figure 5: Task performance differences between SMA and aINS neurofeedback (session 1). Group differences in the first session between the constant task group (aINS neurofeedback, emotion regulation, magenta) and the changing task group (SMA neurofeedback, motor imagery, green). A. Absolute distance to target levels and B. gradual up-regulation of the neurofeedback signal with increasing target level on group level and C. for individual participants. Across all measures of task performance, the changing task group showed superior performance in the first session within the current sample. Error bars depict the standard error of the mean.

\subsection{Differences between training styles}

\subsubsection{Consistency of ROIs}

All participants showed overlap between VOls of consecutive sessions (i.e. session 1 and 2; session 2 and 3), 9/10 participants showed overlap across all sessions and the groups did not differ significantly with regard to percentage overlap of voxel for 
all possible combinations of VOIs: Session 1 \& 2, Session 1 \& 3, Session 2 \& 3, and across all sessions (Table 5).

\begin{tabular}{|c|c|c|c|c|}
\hline Participant & \multicolumn{4}{|c|}{$\%$ Overlap } \\
\hline CON & $1 \& 2$ & $1 \& 3$ & $2 \& 3$ & \% Total \\
\hline P01 & $24.10 \%$ & $31.23 \%$ & $27.71 \%$ & $13.13 \%$ \\
\hline $\mathrm{P} 03$ & $42.14 \%$ & $28.56 \%$ & $24.79 \%$ & $16.12 \%$ \\
\hline P05 & $10.91 \%$ & $0.00 \%$ & $0.06 \%$ & $0.00 \%$ \\
\hline P06 & $34.05 \%$ & $23.53 \%$ & $22.23 \%$ & $12.28 \%$ \\
\hline $\mathrm{P} 10$ & $26.21 \%$ & $29.93 \%$ & $37.71 \%$ & $16.00 \%$ \\
\hline Mean & $27.48 \%$ & $22.65 \%$ & $22.50 \%$ & $\mathbf{1 1 . 5 1 \%}$ \\
\hline \multicolumn{5}{|l|}{ CHA } \\
\hline P02 & $34.11 \%$ & $20.02 \%$ & $22.23 \%$ & $11.09 \%$ \\
\hline P04 & $29.23 \%$ & $17.80 \%$ & $28.29 \%$ & $10.51 \%$ \\
\hline P07 & $12.60 \%$ & $30.34 \%$ & $8.32 \%$ & $4.43 \%$ \\
\hline P08 & $9.43 \%$ & $31.52 \%$ & $8.19 \%$ & $3.96 \%$ \\
\hline P09 & $26.63 \%$ & $12.49 \%$ & $26.57 \%$ & $8.12 \%$ \\
\hline Mean & $22.40 \%$ & $22.43 \%$ & $18.72 \%$ & $7.62 \%$ \\
\hline$p$-value & 0.50 & 0.98 & 0.63 & 0.28 \\
\hline t-value & 0.74 & 0.03 & 0.50 & 1.17 \\
\hline
\end{tabular}

Table 5: Consistency of neurofeedback target regions over time during alNS level modulation: Three-dimensional volume representations of neurofeedback target-regions showed considerable overlap within each participant and across groups over the neurofeedback training. The table contains the positions and cluster extensions of voxelcluster shared between all alNS target-level modulation runs for individual participants and across groups in Talairach space.

\subsubsection{Regulation of the neurofeedback signal}

ANOVA of absolute distance in the second and third session (Figure $6 \mathrm{~A}$ ) revealed no significant group difference $(\mathrm{CHA}$ mean $=4.11, \mathrm{SE}=0.12 ; \mathrm{CON}$ mean $=4.12, \mathrm{SE}$ $=0.12 ; F(1,8)=0.02, p=0.96)$ but participants improved significantly over time pooled across both groups (Session 2 mean $=4.28, \mathrm{SE}=0.09$; Session 3 mean $=3.95$, $\mathrm{SE}=0.08 ; F(1,8)=11.78, p=0.02)$ and the interaction between GROUP $*$ TIME was not significant $(F(1,8)=0.163, p=0.70)$. On the level of individual participants, results were supportive for group findings: 9/10 participants showed lower mean 
absolute distance to the target level in the third session compared to the second session (Figure 6 B).

ANOVA on neurofeedback magnitude in the second and third session (Figure 7) revealed a significant difference between groups ( $\mathrm{CHA}$ mean $=3.63, \mathrm{SE}=0.25 ; \mathrm{CON}$ mean $=2.63, \mathrm{SE}=0.25 ; \mathrm{F}(1,8)=8.16, p=0.02)$, no significant main effect for session $($ Session 2 mean $=2.78, \mathrm{SE}=0.32 ;$ Session 3 mean $=3.48, \mathrm{SE}=0.22 ; F(1,8)=2.7, \mathrm{p}$ $=0.14$ ), no significant difference between target levels (Low target level mean = $3.21, \mathrm{SE}=0.24$; Medium target level mean $=3.18, \mathrm{SE}=0.22$; High target level mean $=3.00, \mathrm{SE}=0.19 ; \mathrm{F}(2,16)=0.49, \mathrm{p}=0.62$ ) and no significant interactions (GROUP * SESSION: $F(1,8)=2.56, p=0.15$; GROUP * TARGET LEVEL: $F(2,16)=0.32, p=0.73$; SESSION * TARGET LEVEL: $F(2,16)=3.44, p=0.06 ;$; GROUP * SESSION * TARGET LEVEL: $F(2,16)=0.28, p=0.97)$. Single-trial analysis results were reflective of the low gradual up-regulation performance across groups, although both groups showed a slight improvement over time (Session2: CON mean $r_{z}=-0.07$; CHA mean $r_{z}=-0.11$; Session3: CON mean $r_{z}=0.04 ;$ CHA mean $r_{z}=-0.01$ ). 


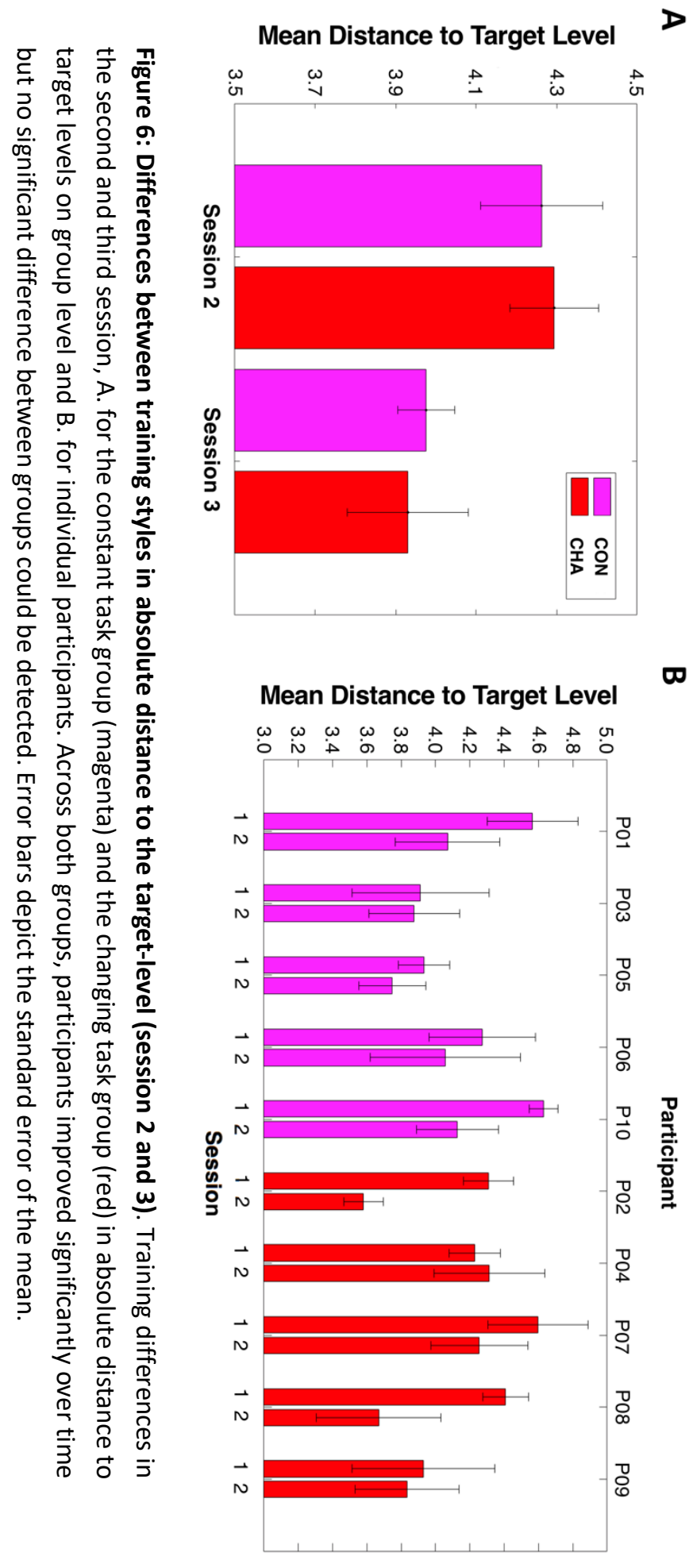


To investigate how the group difference in mean neurofeedback magnitude was related to task performance, negative deviations from the target level (neurofeedback magnitude lower than the target level) and positive deviations from the target level (neurofeedback magnitude exceeding the target level) were compared separately between groups across sessions. The CHA group showed on average a higher ability to reach the target level (mean negative deviations from the target level: $\mathrm{CHA}$ mean $=-2.74, \mathrm{SE}=0.18 ; \mathrm{CON}$ mean $=-3.23, \mathrm{SE}=0.12 ; \mathrm{p}=$ 0.14 ), but tended to exceed the target level (mean positive deviations from the target level: $\mathrm{CHA}$ mean $=1.37, \mathrm{SE}=0.97 ; \mathrm{CON}$ mean $=0.90, \mathrm{SE}=0.15 ; \mathrm{p}=0.06$ ), although the differences were not significant between groups. 

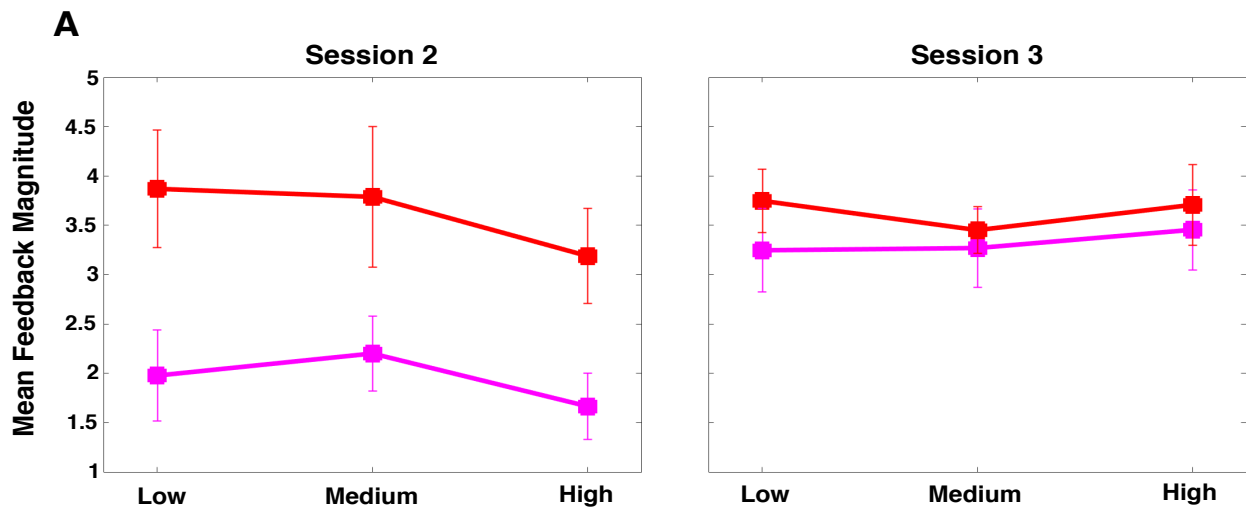

Target Level

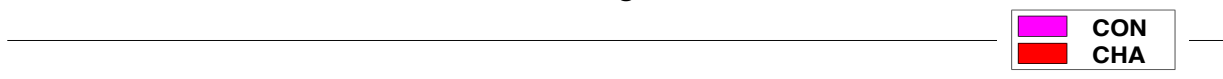

B

Participant

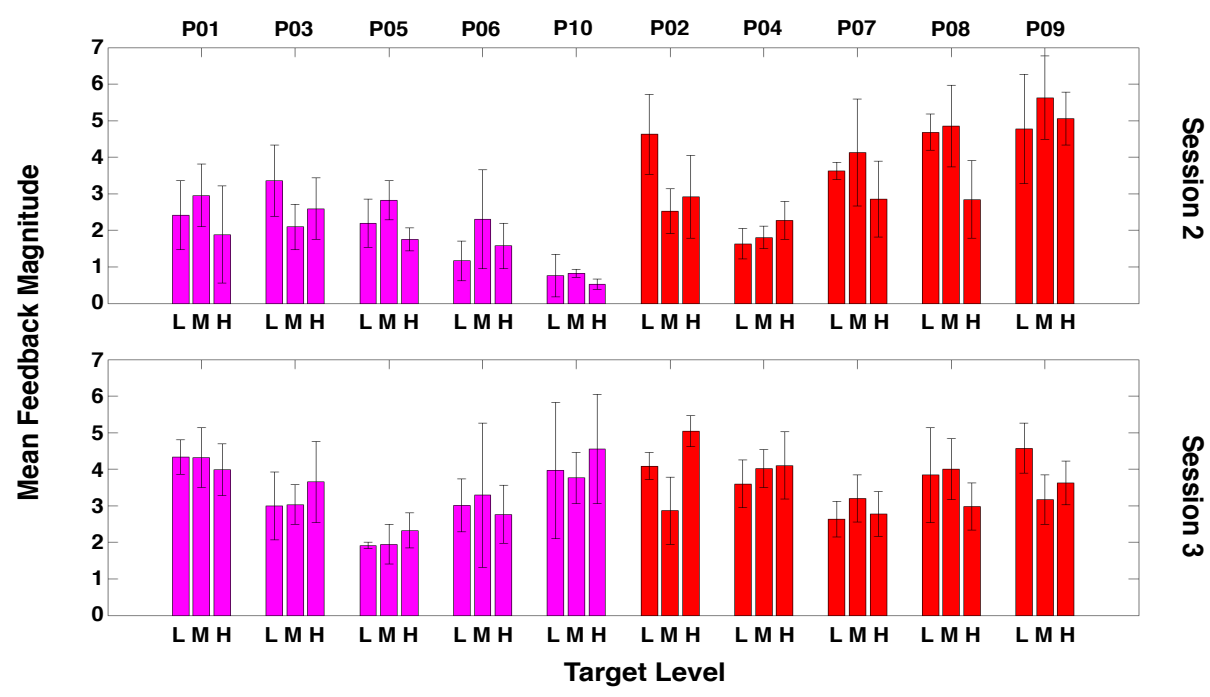

Figure 7: Differences in gradual up-regulation of the aINS neurofeedback signal (session 2 and 3). Training differences in the second and third session, A. for the constant group (magenta) and the changing task group (red) in gradual up-regulation of the neurofeedback signal on group level and B. for individual participants Across both groups, participants failed to up-regulate the signal in accordance to the height of the target level but the changing task group showed a significantly higher mean magnitude of the neurofeedback signal across session (significant main effect), which was driven by significant group difference during session 2. Error bars depict the standard error of the mean. 


\subsubsection{Neurofeedback regulation and positive affect}

Both groups did not show an pronounced trend increase in self-ratings of positive affect over the course of training (Figure 8 A, PANAS positive, Session 1: CHA mean $=33.60, \mathrm{SE}=1.75 ; \mathrm{CON}$ mean $=33.40, \mathrm{SE}=1.89 ;$ Session 2 : $\mathrm{CHA}$ mean $=35.6, \mathrm{SE}=$ 2.34; $\mathrm{CON}$ mean $=29.60, \mathrm{SE}=3.08 ;$ Session 3: $\mathrm{CHA}$ mean $=31.00, \mathrm{SE}=3.83 ; \mathrm{CON}$ mean $=29.80, \mathrm{SE}=2.67$ and the groups did not differ significantly after the training $(p=0.61)$. At the end of the training, up-regulation of the alNS was associated with increased positive affect across both groups $(r=0.65, p=0.04$; Figure 8 B). Correlation analysis between final aINS neurofeedback task performance and positive affect after the training revealed no pronounced relationship between absolute distance to target level and positive affect after the training across both groups $(r=-0.46, p=0.17$; Figure $8 \mathrm{C}$ ). When separating both groups, the two groups showed an inverse relationship between absolute distance with task performance ( $\mathrm{CHA} r=-0.93, p=0.02 ; \mathrm{CON} r=0.94, p=0.01$; Figure $8 \mathrm{D})$. The difference between correlation coefficients was significant $(p=0.001)$.

To increase the neurofeedback signal, both groups shared the two most prevalent categories of mental content for emotion regulation: Focussing on the experience of positive affect (9/10) and interoception (6/10), participants from both groups focussed on olfactory experiences (3/10), while only participants of the $\mathrm{CHA}$ group additionally focussed on somatosensory experiences (3/5) and one participant in the CON group additionally focussed on gustatory experiences (Table 6). Overall, participants in the $\mathrm{CHA}$ group could report more content modalities to increase the neurofeedback signal $(\mathrm{CHA}$ mean $=13, \mathrm{SE}=0.40 ; \mathrm{CON}$ mean $=9, \mathrm{SE}=0.58)$ and the individual strategy rated as most effective for increasing the signal was indicated as being more reliable by participants in the CHA group (Maximal up-regulation effectiveness: $\mathrm{CHA}$ mean $=3.60, \mathrm{SE}=0.25 ; \mathrm{CON}$ mean $=3.20, \mathrm{SE}=0.37$ ), as well as 
the strategy used for decreasing the neurofeedback signal $(\mathrm{CHA}$ mean $=3.20, \mathrm{SE}=$ 0.20 ; $\mathrm{CON}$ mean $=3.00, \mathrm{SE}=0.32$ ). To assess how well participants were able to down-regulate the feedback signal when they exceeded the target level, maximum effectiveness of down-regulation was reported as well. The down-regulation strategies applied by participants were: Distraction (focusing on another mental operation than the mental operation which was used to increase the signal) or disengagement (reducing the mental effort for performing the mental activity).

A

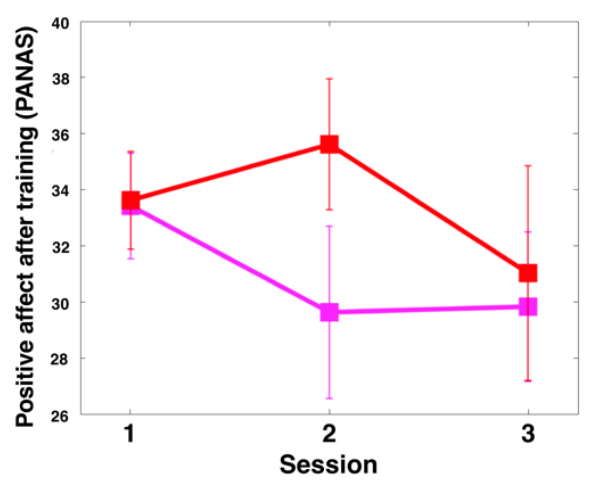

C

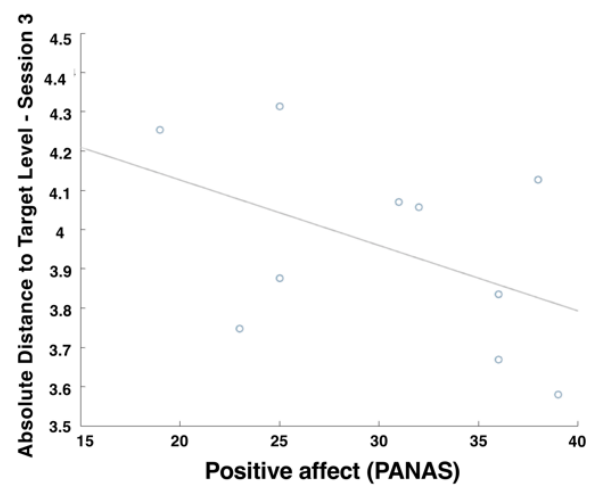

B

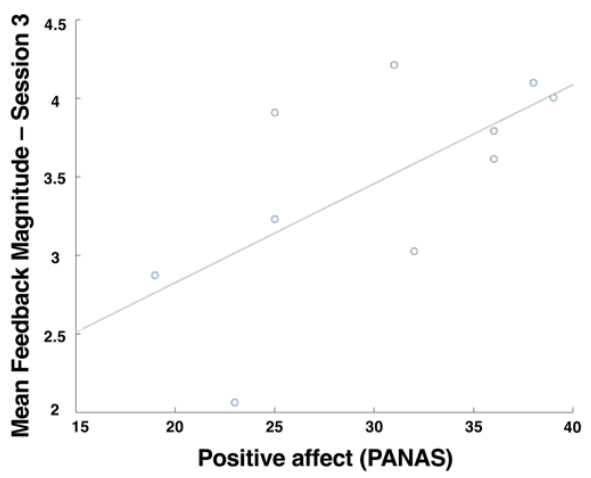

D

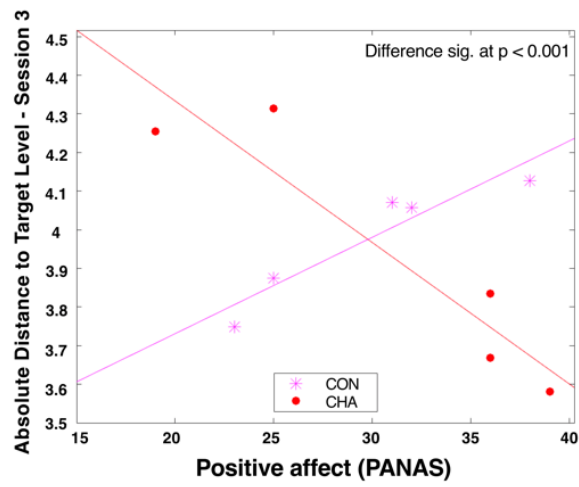

Figure 8: Relationship between positive affect and aINS neurofeedback.

A. Mean ratings in positive affect per group over the course of training reveal no training effect, but marginally higher mean positive affect for the changing task group. B. Relationship between neurofeedback magnitude during the last session and positive affect at the end of training. Up-regulation of the aINS was associated with increased positive 
affect after the training. C. Relationship between absolute distance to target-levels during the last session and positive affect at the end of training across groups. D. Relationship between absolute distance to target levels during the last session and positive affect at the end compared between groups. The changing task group showed a stronger positive relationship between fine grained modulation of the signal and positive affect at the end of training.

\begin{tabular}{|c|c|c|c|c|c|c|c|c|c|}
\hline \multirow{2}{*}{$\begin{array}{c}\text { Participants } \\
\text { CON }\end{array}$} & \multicolumn{5}{|c|}{ Mental Imagery Modality } & & & \multicolumn{2}{|c|}{ Maximal Effectiveness } \\
\hline & $\begin{array}{c}\text { Positive } \\
\text { Affect }\end{array}$ & Olfaction & Gustation & Interoception & Somatosensory & SUM & & Up-regulation & $\begin{array}{c}\text { Down- } \\
\text { regulation }\end{array}$ \\
\hline $\mathrm{P} 01$ & 1 & & & & & 1 & & 3 & 3 \\
\hline P03 & 1 & 1 & 1 & 1 & & 4 & & 2 & 2 \\
\hline P05 & 1 & & & & & 1 & & 3 & 3 \\
\hline P06 & 1 & & & & & 1 & & 4 & 4 \\
\hline $\mathrm{P} 10$ & 1 & & & 1 & & 2 & & 4 & 3 \\
\hline Sum & 5 & 1 & 1 & 2 & $\mathbf{0}$ & 9 & Mean & 3.20 & 3.00 \\
\hline \multicolumn{10}{|l|}{ CHA } \\
\hline $\mathrm{P} 02$ & 1 & 1 & & 1 & 1 & 4 & & 4 & 3 \\
\hline $\mathrm{P} 04$ & 1 & 1 & & & 1 & 3 & & 4 & 4 \\
\hline P07 & & & & 1 & 1 & 2 & & 3 & 3 \\
\hline P08 & 1 & & & 1 & & 2 & & 3 & 3 \\
\hline P09 & 1 & & & 1 & & 2 & & 4 & 3 \\
\hline Sum & 4 & 2 & $\mathbf{0}$ & 4 & 3 & 13 & Mean & 3.60 & 3.20 \\
\hline Overall sum & 9 & 3 & 1 & 6 & 3 & 22 & Mean & 3.40 & 3.10 \\
\hline
\end{tabular}

Table 6: Mental strategies applied for aINS modulation during the final training session. The table summarizes all mental imagery modalities applied by participants for upregulating the aINS during the final training session and reliability ratings of strategies that were rated as being most efficient for up- or down regulating the neurofeedback signal.

\subsubsection{Task-general psychological measures}

Only the CHA group showed a trend increase in motivation (QCM, Session 1: $\mathrm{CHA}$ mean $=4.40, \mathrm{SE}=0.19 ; \mathrm{CON}$ mean $=4.45, \mathrm{SE}=0.45 ;$ Session 2: $\mathrm{CHA}$ mean $=4.63$, $\mathrm{SE}=0.17 ; \mathrm{CON}$ mean $=4.48, \mathrm{SE}=0.37$; Session $3: \mathrm{CHA}$ mean $=4.71, \mathrm{SE}=0.16 ; \mathrm{CON}$ mean $=4.38, \mathrm{SE}=0.38)$. Both groups showed a trend increase in self-rated neurofeedback skills (NSQ quantitative scale, Session 1: CHA mean = 3.17, SE =0.16; $\mathrm{CON}$ mean $=3.00, \mathrm{SE}=0.17$; Session $2: \mathrm{CHA}$ mean $=3.61, \mathrm{SE}=0.18 ;$ mean $=3.08$, $\mathrm{SE}=0.21$; Session 3: $\mathrm{CHA}$ mean $=3.72, \mathrm{SE}=0.12$; $\mathrm{CON}$ mean $=3.38, \mathrm{SE}=0.28)$. Over the course of training, differences between both groups got more pronounced 
(Figure $9 \mathrm{~A}, \mathrm{~B}$ ), but differences between groups were not significant at the end of training (QCM: $p=0.54 ; N S Q$, quantitative scale: $p=0.10$ ).
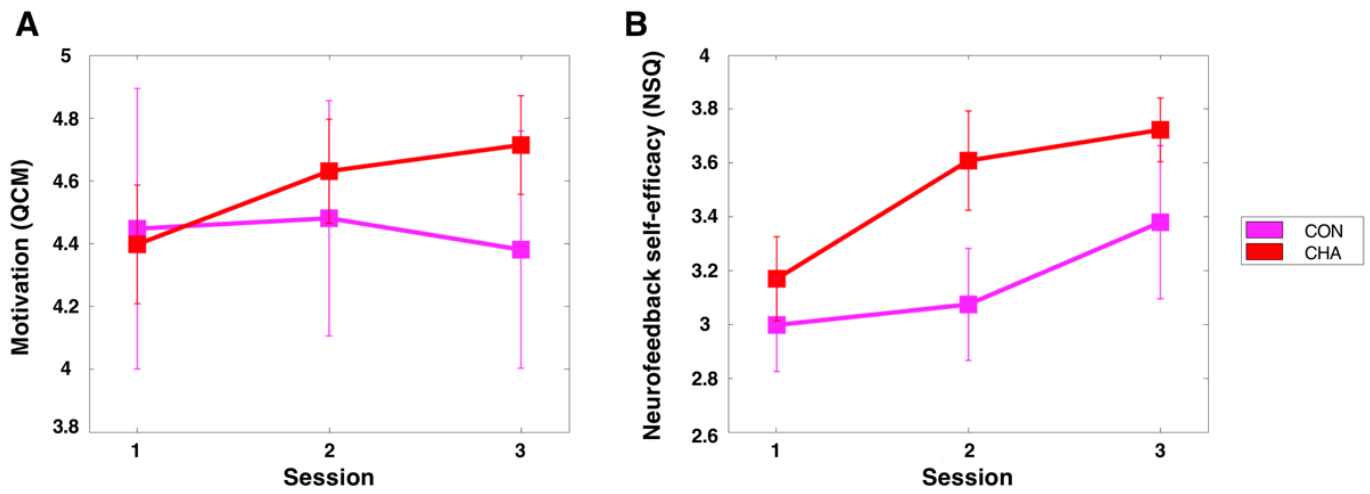

Figure 9: Changes in general psychological measures of interest over the training. Mean ratings in general psychological measures of interest over the course of training compared between groups. A. Mean ratings in motivation: The changing task group showed slightly increased motivation at the end of training. B. Neurofeedback self-efficacy: Both groups increased in neurofeedback self-efficacy over the course of training, while the changing task group showed higher self-efficacy during all sessions.

\section{Discussion}

The current project aimed to determine whether step-wise increases in task difficulty can be beneficial for the outcome of neurofeedback trainings, by comparing neurofeedback performance between two different training styles, training with a constant task in comparison to training with changing tasks and difficulty levels. In the present study, both groups trained to regulate activation in the aINS to three different target levels. Gradual up-regulation ability, the relation between neurofeedback and the psychological target process, positive affect, and differences in task-general psychological measures were evaluated. 


\subsection{Differences in task difficulty between neurofeedback modalities}

To understand whether we succeeded in providing the $\mathrm{CHA}$ group with a lower level of task difficulty as starting point, we compared the ability to stabilize the neurofeedback signal around three target levels across the two initial neurofeedback tasks, which differed with regard to self-regulation modality between groups: motor-imagery-guided by SMA neurofeedback for the CHA group and positive emotion regulation to modulate aINS activation for the CON group (Figure 5).

Within the current sample, control over SMA neurofeedback was achieved more reliably by participants than control over aINS neurofeedback. The CHA group was able to up-regulate the neurofeedback more strongly, while stabilizing it more accurately around target levels, compared to the CON group. While the CHA group was able to significantly increase the SMA neurofeedback in a linear fashion according to the target levels, the CON group failed to do so for the aINS.

While several previous studies have demonstrated successful regulation of the aINS in the sense of increasing or decreasing its activation through emotion regulation guided by neurofeedback (Caria et al., 2007; Johnston et al., 2011; Zilverstand et al., 2015), this is to our knowledge the first study that describes attempted finegrained modulation on alNS activation through neurofeedback. In turn, SMA neurofeedback has already been tested for target-level neurofeedback and previous studies demonstrated that participants are able to modulate SMA activation to different target activation levels when provided with neurofeedback (Mehler et al., 2019; Sorger et al., 2016). 


\subsection{Effects of training styles}

\subsubsection{Effects of training styles on neurofeedback regulation}

The groups did not differ significantly with regard to absolute distance-to-target level or ability to differentiate between target levels (Figure 6). Over the course of training, both groups significantly decreased the absolute distance to the target level, suggesting that both groups learned to interact with the feedback in a meaningful way. As the task performance was considerably low across both groups, the failure to detect a difference in overall task performance might have been influenced by a floor effect caused by the complexity of the neurofeedback task, as well as by low statistical power due to the limited sample size.

To explore in more detail, whether the two groups showed differences in their selfregulation behaviour, we investigated the tendency to up-regulate the feedback signal, i.e., whether participants were able to identify a mental strategy that increased activation in the aINS in comparison to baseline, the ability to reach / hold activation at the target level, as well as the ability to not exceed the target level.

Analysis of the mean feedback magnitude revealed that participants in the $\mathrm{CHA}$ group significantly increased the neurofeedback signal more strongly than the CON group across both sessions, mainly during session 2 (Figure 7). Post-hoc analysis on feedback magnitude in relation to the target level revealed a trend for the $\mathrm{CHA}$ group to reach / hold the target level more adequately, but also to overshoot. Overall, these group differences suggest that participants in the CHA group identified more effective strategies to increase the signal, thereby facing more difficulties in stabilizing the signal around the target level. This notion is in accordance with changes in positive affect ratings over time (Figure $8 \mathrm{~A}$ ). 


\subsubsection{Effects of straining styles on positive affect}

To investigate the efficacy of the neurofeedback training with regard to emotional self-regulation, we analysed the relationship between magnitude of the neurofeedback signal, neurofeedback performance and positive affect scores after training (Figure 8). Rating scores alone were not reflective of training differences or changes over time. But positive affect after the training was tied to the magnitude of the neurofeedback: A higher mean magnitude of the neurofeedback signal during the last session was associated with increased positive affect after the training. This relationship supports that the neurofeedback signal indeed reflected positive emotional states and is in accordance with a recent large scale study on endogenous generation of positive affect by Engen, Kanske, and Singer (2016). Here, higher valence (positive or negative), correlated with alNS activation.

Additionally, only the $\mathrm{CHA}$ group showed a stronger positive correlation between neurofeedback task performance (i.e. reaching and holding a target activation level) and positive affect after the training. On the one hand, this could suggest that gaining control over the neurofeedback signal was experienced as more rewarding for the CHA group, leading to mood improvements after the training. On the other hand, these findings could also reflect that both groups developed different emotion regulation styles with different effects on the emotional system. A more thorough analysis on neurofeedback performance supported that both groups showed slightly different self-regulation behaviour: The CHA grouped tended to exceed the target level more often than the CON group, i.e. participants engaged more strongly in positive emotional states than needed to reach the target level. The CON group in turn tended to engage less in positive emotions than needed to reach/hold a target level compared to the CHA group. Furthermore, participants in the $\mathrm{CHA}$ group identified more strategies to up-regulate the neurofeedback signal 
and rated their most effective up- or down regulation strategies as more reliable than the CON group (Table 6). However, considering the sample size of this proof of concept study, the correlation differences are exploratory and have to be validated in further research.

\subsubsection{Effects of straining styles on task general psychological processes}

To understand whether differences in training outcomes were reflected in differences in task-general psychological variables, we compared self-ratings on neurofeedback self-efficacy and motivation over the course of training on a descriptive level (Figure 9), taking into account the sample size. Overall, participants showed a general increase in self-efficacy over time, as in accordance with the observed improvements in task performance across groups, while only the CHA group increased in motivation over time. Comparing both groups, the CHA group showed higher neurofeedback self-efficacy during all sessions and a growing trend of increased motivation over time, with the group difference being most pronounced after the last session. Overall, results are reflective of the observed learning effect and suggest a slight advantage for starting the learning process with a less demanding initial task, although group differences were not significant at the end of training.

\subsection{Implications and future directions}

The paradigm of the current study revealed to be very challenging for participants: Although learning effects were present, participants were not able to modulate the aINS neurofeedback signal in accordance with target levels. This is likely due to the choice of the region of interest, as the aINS is part of the network generally involved 
in neurofeedback and activation in the aINS has repeatedly been demonstrated to show increased activation during neurofeedback based self-regulation, across different mental tasks (Emmert et al., 2016) and unintentional increases in aINS activation can therefore be expected during neurofeedback guided self-regulation. The prevalence of group differences in detecting an effective strategy to upregulate the neurofeedback region, supported by the observed relation between neurofeedback magnitude and positive affect, self-regulation strategies, motivation and self-efficacy, suggest that the CHA group benefited more strongly from the training in this challenging task environment. The significantly more positive correlation between neurofeedback performance and positive affect additionally supports, that for the CHA group the reward provided by neurofeedback had been related to the psychological target process: Positive affect. Taken into account the considerably small sample size from which the results were acquired, expected effects were small. Nevertheless, the current effects were consistent across different markers of performance and thereby provide converging evidence across participants.

While in our study, the $\mathrm{CHA}$ group received a different neurofeedback tasks in the begin of the training, providing other (non-neurofeedback) self-regulation tasks before the neurofeedback training could possibly also support neurofeedback performance. For example, general cognitive self-regulation training or training with other biofeedback modalities could be used to improve training schemes further, but systematic analyses are pending.

Considering that many different forms of self-regulation could in theory be combined to facilitate treatment effects, the question which mental mechanisms are transferred between different forms of self-regulation arises. On the one hand, 
it has been shown that neurofeedback guided self-regulation shares a common neural basis with other forms of self-regulation in executive control regions (Emmert et al., 2016; Marchesotti et al., 2017; Sitaram et al., 2017) and regions related to introspection, notably the anterior insula. General skills in self-regulation could therefore potentially be trained and transferred from one self-regulation method to another. Interestingly in the context of the current training, only participants from the $\mathrm{CHA}$ group reported to focus on somatosensory experience during the final emotion regulation training session. While somatosensory experience of imagined movements was implicated in the motor imagery localizer for both groups, only the CHA group performed extensive motor imagery during the first session, thereby potentially broadening the search space for finding effective mental strategies for emotion regulation later on.

While some neurofeedback studies have succeeded in helping participants to transfer skills acquired during neurofeedback to self-regulation without neurofeedback (Auer, Schweizer, \& Frahm, 2015; Nicholson et al., 2017) or even to different tasks (Gevensleben et al., 2014; Zhang, Yao, Zhang, Long, \& Zhao, 2013), understanding the psychological basis of transfer mechanisms can be crucial for establishing long lasting effects of neurofeedback interventions. In this context, the results of the current study support a broader scope on analysing and designing neurofeedback interventions, by not only focusing on the psychological targetprocesses or its neural reflection, but also taking the transferable aspects of selfregulation into account. 


\subsection{Conclusion}

Overall, the current study extended the field by showing that providing participants with a different initial task with lower task difficulty can increase the effectiveness of a neurofeedback training. While in general, longer neurofeedback interventions can be expected to show more profound training outcomes, both groups in the current study spend the exact same amount of time in the MRI environment. Designs including an initial neurofeedback task with lower task difficulty should therefore be considered for increasing the cost efficacy of neurofeedback trials, by speeding the learning process. However, the current design is just a first proof of concept and future studies are needed to determine the most effective design, by examining different mental tasks and levels of task difficulty. 


\section{References}

Alkoby, O., Abu-Rmileh, A., Shriki, O., \& Todder, D. (2017). Can we predict who will respond to neurofeedback? A review of the inefficacy problem and existing predictors for successful EEG neurofeedback learning. Neuroscience.

Auer, T., Schweizer, R., \& Frahm, J. (2015). Training efficiency and transfer success in an extended real-time functional MRI neurofeedback training of the somatomotor cortex of healthy subjects. Frontiers in human neuroscience, 9, 547.

Blanchard, E. B., \& Young, L. D. (1974). Clinical applications of biofeedback training: A review of evidence. Archives of General Psychiatry, 30(5), 573-589.

Borm, G. F., Hoogendoorn, E. H., den Heijer, M., \& Zielhuis, G. A. (2005). Sequential balancing: a simple method for treatment allocation in clinical trials. Contemporary clinical trials, 26(6), 637-645.

Bouchard, S., Gauthier, J., Nouwen, A., Ivers, H., Vallières, A., Simard, S., \& Fournier, T. (2007). Temporal relationship between dysfunctional beliefs, self-efficacy and panic apprehension in the treatment of panic disorder with agoraphobia. Journal of Behavior Therapy and Experimental Psychiatry, 38(3), 275-292.

Burleson, J. A., \& Kaminer, Y. (2005). Self-efficacy as a predictor of treatment outcome in adolescent substance use disorders. Addictive behaviors, 30(9), 1751-1764.

Caria, A., Veit, R., Sitaram, R., Lotze, M., Weiskopf, N., Grodd, W., \& Birbaumer, N. (2007). Regulation of anterior insular cortex activity using real-time fMRI. Neurolmage, 35(3), 1238-1246.

Dalkner, N., Unterrainer, H. F., Wood, G., Skliris, D., Holasek, S. J., Gruzelier, J. H., \& Neuper, C. (2017). Short-term Beneficial Effects of 12 Sessions of Neurofeedback on Avoidant Personality Accentuation in the Treatment of 
Alcohol Use Disorder. Frontiers in psychology, 8, 1688.

Daum, I., Rockstroh, B., Birbaumer, N., Elbert, T., Canavan, A., \& Lutzenberger, W. (1993). Behavioural treatment of slow cortical potentials in intractable epilepsy: neuropsychological predictors of outcome. Journal of Neurology, Neurosurgery \& Psychiatry, 56(1), 94-97.

Diaz, R. M., Winsler, A., Atencio, D. J., \& Harbers, K. (1992). Mediation of selfregulation through the use of private speech. International Journal of Cognitive Education and Mediated Learning, 2(2), 155-167.

Eid, M., Gollwitzer, M., \& Schmitt, M. (2010). Statistik und Forschungsmethoden (pp. 548-549).

Emmert, K., Breimhorst, M., Bauermann, T., Birklein, F., Van De Ville, D., \& Haller, S. (2014). Comparison of anterior cingulate vs. insular cortex as targets for real-time fMRI regulation during pain stimulation. Frontiers in behavioral neuroscience, 8, 350.

Emmert, K., Kopel, R., Sulzer, J., Brühl, A. B., Berman, B. D., Linden, D. E., . . Frank, S. (2016). Meta-analysis of real-time fMRI neurofeedback studies using individual participant data: How is brain regulation mediated? Neurolmage, $124,806-812$.

Engen, H. G., Kanske, P., \& Singer, T. (2016). The neural component-process architecture of endogenously generated emotion. Social cognitive and affective neuroscience, 12(2), 197-211.

Freund, P. A., Kuhn, J.-T., \& Holling, H. (2011). Measuring current achievement motivation with the QCM: Short form development and investigation of measurement invariance. Personality and Individual Differences, 51(5), 629-634.

Gallagher, M. W., Payne, L. A., White, K. S., Shear, K. M., Woods, S. W., Gorman, J. M., \& Barlow, D. H. (2013). Mechanisms of change in cognitive behavioral 
therapy for panic disorder: the unique effects of self-efficacy and anxiety sensitivity. Behaviour research and therapy, 51(11), 767-777.

Gevensleben, H., Kleemeyer, M., Rothenberger, L. G., Studer, P., Flaig-Röhr, A., Moll, G. H., ... Heinrich, H. (2014). Neurofeedback in ADHD: further pieces of the puzzle. Brain topography, 27(1), 20-32.

Ghosh, T., Jahan, M., \& Singh, A. R. (2014). The efficacy of electroencephalogram neurofeedback training in cognition, anxiety, and depression in alcohol dependence syndrome: A case study. Industrial psychiatry journal, 23(2), 166.

Goldin, P. R., Ziv, M., Jazaieri, H., Werner, K., Kraemer, H., Heimberg, R. G., \& Gross, J. J. (2012). Cognitive reappraisal self-efficacy mediates the effects of individual cognitive-behavioral therapy for social anxiety disorder. Journal of Consulting and Clinical Psychology, 80(6), 1034.

Guo, J., Zhao, X., Li, Y., Yao, L., \& Chen, K. (2012). Primary motor cortex activity reduction under the regulation of SMA by real-time fMRI. Paper presented at the Medical Imaging 2012: Biomedical Applications in Molecular, Structural, and Functional Imaging.

Hammond, D. C. (2005). Neurofeedback treatment of depression and anxiety. Journal of Adult Development, 12(2-3), 131-137.

Hanlon, C. A., Hartwell, K. J., Canterberry, M., Li, X., Owens, M., LeMatty, T., . . . George, M. S. (2013). Reduction of cue-induced craving through realtime neurofeedback in nicotine users: the role of region of interest selection and multiple visits. Psychiatry Research: Neuroimaging, 213(1), 79-81.

Hevey, D., Smith, M. I., \& McGee, H. M. (1998). Self-efficacy and health behaviour: a review. The Irish Journal of Psychology, 19(2-3), 248-273.

Johnston, S., Linden, D., Healy, D., Goebel, R., Habes, I., \& Boehm, S. (2011). Upregulation of emotion areas through neurofeedback with a focus on 
positive mood. Cognitive, Affective, \& Behavioral Neuroscience, 11(1), 4451.

Kurth, F., Zilles, K., Fox, P. T., Laird, A. R., \& Eickhoff, S. B. (2010). A link between the systems: functional differentiation and integration within the human insula revealed by meta-analysis. Brain Structure and Function, 214(5-6), 519-534.

Lang, P. J., Bradley, M. M., \& Cuthbert, B. N. (1997). International affective picture system (IAPS): Technical manual and affective ratings. NIMH Center for the Study of Emotion and Attention, 39-58.

Linden, D. E. (2014). Neurofeedback and networks of depression. Dialogues in clinical neuroscience, 16(1), 103.

Loeber, S., Croissant, B., Heinz, A., Mann, K., \& Flor, H. (2006). Cue exposure in the treatment of alcohol dependence: Effects on drinking outcome, craving and self-efficacy. British Journal of Clinical Psychology, 45(4), 515-529.

Marchesotti, S., Martuzzi, R., Schurger, A., Blefari, M. L., del Millán, J. R., Bleuler, H., \& Blanke, O. (2017). Cortical and subcortical mechanisms of brain-machine interfaces. Human Brain Mapping, 38(6), 2971-2989.

Mehler, D. M., Sokunbi, M. O., Habes, I., Barawi, K., Subramanian, L., Range, M., . . . Keedwell, P. (2018). Targeting the affective brain-a randomized controlled trial of real-time fMRI neurofeedback in patients with depression. Neuropsychopharmacology, 1.

Mehler, D. M., Williams, A. N., Krause, F., Lührs, M., Wise, R. G., Turner, D. L., . . . Whittaker, J. R. (2019). The BOLD response in primary motor cortex and supplementary motor area during kinesthetic motor imagery based graded fMRI neurofeedback. Neurolmage, 184, 36-44.

Mitchell, C., \& Stuart, R. B. (1984). Effect of self-efficacy on dropout from obesity treatment. Journal of Consulting and Clinical Psychology, 52(6), 1100.

Moore, N. C. (2000). A review of EEG biofeedback treatment of anxiety disorders. 
Clinical electroencephalography, 31(1), 1-6.

Nicholson, A. A., Rabellino, D., Densmore, M., Frewen, P. A., Paret, C., Kluetsch, R., ... McKinnon, M. C. (2017). The neurobiology of emotion regulation in posttraumatic stress disorder: Amygdala downregulation via real-time fMRI neurofeedback. Human Brain Mapping, 38(1), 541-560.

Nijboer, F., Birbaumer, N., \& Kubler, A. (2010). The influence of psychological state and motivation on brain-computer interface performance in patients with amyotrophic lateral sclerosis-a longitudinal study. Frontiers in neuroscience, 4, 55.

Nijboer, F., Furdea, A., Gunst, I., Mellinger, J., McFarland, D. J., Birbaumer, N., \& Kübler, A. (2008). An auditory brain-computer interface (BCI). Journal of neuroscience methods, 167(1), 43-50.

Porter, J. M., \& Saemi, E. (2010). Moderately Skilled Learners Benefit by Practicing with Systematic Increases in Contextual Interference. International Journal of Coaching Science, 4(2).

Quaedflieg, C. W., Smulders, F. T., Meyer, T., Peeters, F., Merckelbach, H., \& Smeets, T. (2015). The validity of individual frontal alpha asymmetry EEG neurofeedback. Social cognitive and affective neuroscience, 11(1), 33-43.

Rance, M., Ruttorf, M., Nees, F., Schad, L. R., \& Flor, H. (2014). Real time fMRI feedback of the anterior cingulate and posterior insular cortex in the processing of pain. Human Brain Mapping, 35(12), 5784-5798.

Rostami, R., \& Dehghani-Arani, F. (2015). Neurofeedback training as a new method in treatment of crystal methamphetamine dependent patients: A preliminary study. Applied psychophysiology and biofeedback, 40(3), 151161.

Ryan, R. M., \& Deci, E. L. (2008). A self-determination theory approach to psychotherapy: The motivational basis for effective change. Canadian 
Psychology/Psychologie canadienne, 49(3), 186.

Ryan, R. M., Plant, R. W., \& O'Malley, S. (1995). Initial motivations for alcohol treatment: Relations with patient characteristics, treatment involvement, and dropout. Addictive behaviors, 20(3), 279-297.

Scharnowski, F., Weiskopf, N., Mathiak, K., Zopf, R., Studer, P., Bock, S. W., .. Goebel, R. (2004). Self-regulation of the BOLD signal of supplementary motor area (SMA) and parahippocampal place area (PPA): fMRI-neurofeedback and its behavioural consequences. Neurolmage, 22, e704-e705.

Schneider, F., Rockstroh, B., Heimann, H., Lutzenberger, W., Mattes, R., Elbert, T., .. . Bartels, M. (1992). Self-regulation of slow cortical potentials in psychiatric patients: Schizophrenia. Biofeedback and Self-regulation, 17(4), 277-292.

Sitaram, R., Ros, T., Stoeckel, L., Haller, S., Scharnowski, F., Lewis-Peacock, J., . . . Oblak, E. (2017). Closed-loop brain training: the science of neurofeedback. Nature reviews neuroscience, 18(2), 86.

Sorger, B., Kamp, T., Weiskopf, N., Peters, J. C., \& Goebel, R. (2016). When the brain takes 'BOLD'steps: real-time fMRI neurofeedback can further enhance the ability to gradually self-regulate regional brain activation. Neuroscience.

Sterman, M., \& Friar, L. (1972). Suppression of seizures in an epileptic following sensorimotor EEG feedback training. Electroencephalography and clinical neurophysiology, 33(1), 89-95.

Subramanian, L., Morris, M. B., Brosnan, M., Turner, D. L., Morris, H. R., \& Linden, D. E. (2016). Functional magnetic resonance imaging neurofeedback-guided motor imagery training and motor training for Parkinson's disease: randomized trial. Frontiers in behavioral neuroscience, 10, 111.

Talairach, J., \& Tournoux, P. (1988). Co-planar Stereotaxic Atlas of the Human Brain: 3-dimensional Proportional System : an Approach to Cerebral Imaging: Thieme. 
Van Zundert, M., Könings, K., Sluijsmans, D., \& Van Merriënboer, J. (2012). Teaching domain-specific skills before peer assessment skills is superior to teaching them simultaneously. Educational Studies, 38(5), 541-557.

Wangler, S., Gevensleben, H., Albrecht, B., Studer, P., Rothenberger, A., Moll, G. H., \& Heinrich, H. (2011). Neurofeedback in children with ADHD: specific eventrelated potential findings of a randomized controlled trial. Clinical Neurophysiology, 122(5), 942-950.

Watson, D., Clark, L. A., \& Tellegen, A. (1988). Development and validation of brief measures of positive and negative affect: the PANAS scales. Journal of personality and social psychology, 54(6), 1063.

Weiskopf, N., Mathiak, K., Bock, S. W., Scharnowski, F., Veit, R., Grodd, W., . . . Birbaumer, N. (2004). Principles of a brain-computer interface (BCl) based on real-time functional magnetic resonance imaging (fMRI). IEEE transactions on biomedical engineering, 51(6), 966-970.

Young, K. D., Zotev, V., Phillips, R., Misaki, M., Yuan, H., Drevets, W. C., \& Bodurka, J. (2014). Real-time FMRI neurofeedback training of amygdala activity in patients with major depressive disorder. PloS one, 9(2), e88785.

Zhang, G., Yao, L., Zhang, H., Long, Z., \& Zhao, X. (2013). Improved working memory performance through self-regulation of dorsal lateral prefrontal cortex activation using real-time fMRI. PloS one, 8(8), e73735.

Zilverstand, A., Sorger, B., Sarkheil, P., \& Goebel, R. (2015). fMRI neurofeedback facilitates anxiety regulation in females with spider phobia. Frontiers in behavioral neuroscience, 9, 148. 


\section{Supplementary Material}

\section{SI: The Neurofeedback Skills Questionnaire (NSQ)}

The Neurofeedback Skills Questionnaire (NSQ) was developed to assess selfefficacy and self- regulation skills during neurofeedback trainings. Results are described in Chapter 2. It consists of the following, independently scored scales:

\section{Scale I. Mental Strategies}

\subsection{Summary}

This subscale aims to assess how many mental strategies a participant has explored and how many effective mental strategies a participant has discovered to control the neurofeedback.

It includes open questions regarding the mental content of applied regulation strategies as well as Likert scales that indicate the effectiveness of these strategies to control the neurofeedback signal. Strategies used for up-regulating and for down-regulating the neurofeedback signal are listed separately. For the current study, participants could indicate up to eight regulation strategies.

\subsection{Items}

Please answer the following questions on feedback during emotion regulation.

Please describe as many strategies as you can think of to [increase / decrease] the feedback (if some strategies are similar try to summarize them under one term) and rate how effective this strategy was for you:

Strategy 1: [Empty space for strategy description]

[Does not work - rarely works - works sometimes - works most of the time - works always]

Strategy 2: [Empty space for strategy description]

[Does not work - rarely works - works sometimes - works most of the time - works always] 


\subsection{Scoring}

Firstly, mental strategies are categorized into predefined, theoretically motivated categories, as common practice for qualitative data analysis. Notably, between different neurofeedback studies considerable variability in mental tasks and neurofeedback target regions exists. For this reason, mental strategies applied by participants should be grouped according to existing empirical evidence on the mental processes specific to the given neurofeedback task / target area.

Secondly, the total amount of categories explored by participants should be summed. For example, in the case of aINS in chapter 2 of this thesis categories used to group answers were positive affect, olfaction, gustation, interoception, somatosensory experience. If a participant performed somatosensory imagery (e.g. imagining a pleasant touch) and olfactory imagery (e.g. imagining smelling flowers), this would result in a score of 2 (see Table 6). This value indicates how many mental domains were explored in order to identify an effective strategy.

Thirdly, Likert scores of the mental strategies should be coded as:

Does not work $=0$; Rarely works $=1$; Works sometimes $=2$; Works most of the time $=3$; Works always $=4$.

Fourthly, the maximum Likert scale rating is used as an index on whether participants could identify a strategy that provided full control over the signal or not. 


\section{Scale II. Experienced Control}

\subsection{Summary}

The second scale was constructed to create a comprehensive marker of how participants estimate their ability to control the neurofeedback. Included questions should sample experiences of control in the neurofeedback setting, i.e. self-efficacy (including feeling of control and perceived success) and self-evaluation of acquired knowledge (including whether participants learned strategies to or up-/-down regulate the signal and whether they could predict the temporal delay between their mental actions and feedback changes).

\subsection{Items}

All items are answered with a Likert scale:

[Strongly Disagree - Disagree somewhat - Neutral - Agree somewhat - Agree Strongly]

\subsubsection{Sorted by main categories}

\section{Cluster 1A: Feeling of control}

1A1 When I changed my thoughts the feedback also changed

1 A2 The feedback followed my thoughts

1 A3 The feedback increased when I wanted it to increase

1A4 The feedback decreased when I wanted it to decrease

1A5 I could determine the speed at which the feedback signal would increase

1A6 I could determine the speed at which the feedback signal would decrease

\section{Cluster 1B: Perceived success}

1B1 I was able to control the feedback

1B2 I could reach the lowest target level when I had to

1B3 I could reach the medium target level when I had to

1B4 I could reach the highest target level when I had to

1B5 I could hold the feedback near the lowest target level when I had to

1B6 I could hold the feedback near the medium target level when I had to

1B7 I could hold the feedback near the highest target level when I had to 


\section{Cluster 2A: Mental strategies}

2A1 I would know how to change my thoughts in order to increase the feedback

2A2 I would know how to change my thoughts in order to decrease the feedback

2A3 When the feedback changed, I could explain to myself why this was the case

2A4 The feedback often changed without any obvious reason (Inverse scored)

Cluster 2B: Temporal relationships

2B1 The feedback often changed faster than I expected (Inverse scored)

2B2 The feedback often changed slower than I expected (Inverse scored)

2B3 I could predict how long it would take for the feedback to decrease when I change my thoughts

2B4 I could predict how long it would take for the feedback to increase when I changed my thoughts

\subsubsection{Pseudo-Randomized order}

1 (1A1) When I changed my thoughts the feedback also changed

2 (2B1) The feedback often changed faster than I expected (Inverse scored)

3 (1A3) The feedback increased when I wanted it to increase

4 (1B4) I could reach the highest target level when I had to

5 (1B1) I was able to control the feedback

6 (2B2) The feedback often changed slower than I expected (Inverse scored)

7 (1B5) I could hold the feedback near the lowest target level when I had to

8 (1A5) I could determine the speed at which the feedback signal would increase

9 (1B2) I could reach the lowest target level when I had to

10 (2B3) I could predict how long it would take for the feedback to decrease when I changed my thoughts

11 (1B6) I could hold the feedback near the medium target level when I had to

12 (2A1) I would know how to change my thoughts in order to increase the feedback

13 (1A4) The feedback decreased when I wanted it to decrease

14 (2A2) I would know how to change my thoughts in order to decrease the feedback

15 (1B7) I could hold the feedback near the highest target level when I had to

16 (1A6) I could determine the speed at which the feedback signal would decrease

17 (2A4) The feedback often changed without any obvious reason (Inverse scored) 
18 (1B3) I could reach the medium target level when I had to

19 (1A2) The feedback followed my thoughts

20 (2B4) I could predict how long it would take for the feedback to increase when I changed my thoughts

21 (2A3) When the feedback changed, I could explain to myself why this was the case

\subsection{Scoring}

Likert scores of the mental strategies should be coded as: Strongly Disagree $=1$; Disagree somewhat $=2$; Neutral $=3$; Agree somewhat $=4$; Agree Strongly $=5$. Items 2 (2B1), 6 (2B2) and 17 (2A4) are scored inversely. Finally, values are summed. 


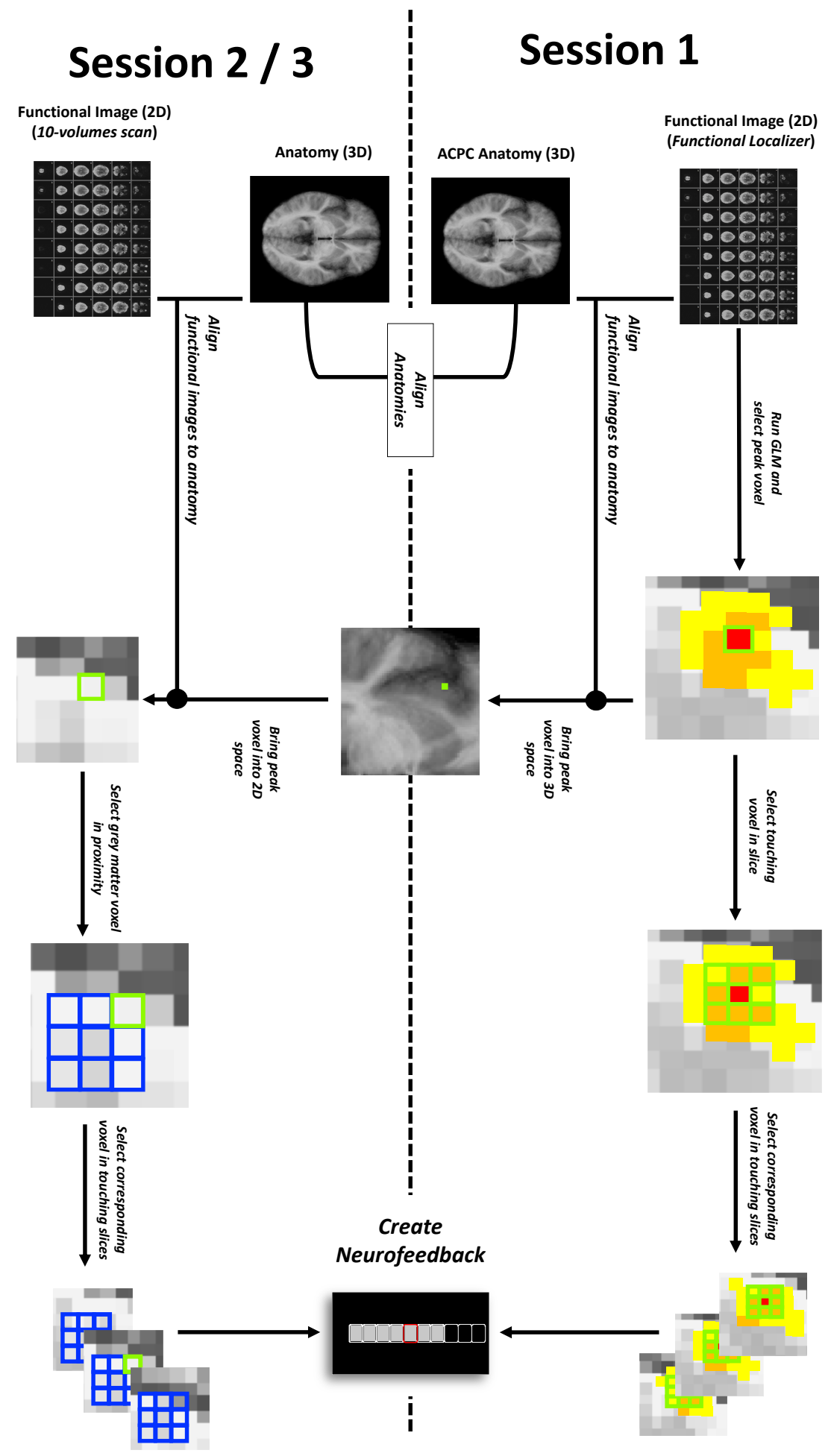


Figure SII: ROI selection procedure. During the first neurofeedback session (top) a general linear model (GLM) was calculated on functional data obtained during the localizer runs and the functional data is aligned to the anatomical data of session one. The peak voxel of the activation cluster in the region of interest (aINS for emotion regulation and SMA for motor imagery) was selected. Subsequently, all voxels touching the peak voxel were selected $(3 * 3$ voxel in the same slice, as well as the corresponding voxels in the slices below and above). The resulting region of $3 * 3 * 3$ voxel constituted the neurofeedback target region of session one. Furthermore, the peak voxel was interpolated to 3-volume space and saved. In the second and third session (bottom) a short resting-state localizer scan was performed to determine the position of functional slices. Anatomies of session two / three were aligned to the anatomical image of session one in ACPC space. Subsequently, functional data was aligned to the anatomical scan of the respective session. Subsequently, the neurofeedback regions were created by selecting $3^{*} 3^{*} 3$ voxel in direct proximity to the central voxel corresponding to the aINS or SMA (i.e. selecting grey matter voxels). 




\section{Chapter 3 - A stable network that adapts flexibly: Whole-brain activation and target-region connectivity during rtfMRI neurofeedback across different mental tasks and time}

Based on:

Skottnik, L., Sorger, B., Benjamins, C., \& Goebel, R. (in preparation). A stable network that adapts flexibly: Wholebrain activation and target-region connectivity during rtfMRI neurofeedback across different mental tasks and time. 



\section{Abstract}

Although neurofeedback has been applied in a broad variety of clinical and research settings, comparably little is known about changes induced by neurofeedback beyond the neurofeedback target region, especially with regard to changes in brain connectivity. To contribute to this issue, we examined the neural basis of neurofeedback guided self-regulation using two different fMRI markers of functional processing: Activation and instantaneous connectivity of the neurofeedback target region. To test the stability of involved networks across different neurofeedback tasks and time, ten participants underwent three realtime fMRI neurofeedback training sessions, in order to learn to regulate activation of the anterior insula (aINS) to three different target levels, either by performing the identical emotion regulation task over the whole training, or by first training motor imagery with neurofeedback from the supplementary motor area (SMA) during session one. By comparing brain activation between the two different mental tasks, we could replicate recent findings on the involvement of a domaingeneral network in neurofeedback, while additionally revealing differential activation in task-specific regions selectively involved in emotion regulation and motor imagery. Across both neurofeedback tasks, neurofeedback target regions showed significant increases in connectivity that overlapped with brain activation shared between both tasks, supporting the domain general function of the involved network. During subsequent sessions, activation and connectivity remained largely constant. Differences between sessions where observed for activation and connectivity of default-mode network nodes and the anterior striatum. These results demonstrate that during neurofeedback a stable network is recruited, that adapts to different mental tasks and undergoes subtle changes over time, supporting the potential of neurofeedback as a reliable tool for research and clinical interventions. 


\section{Introduction}

During the last decade, a growing body of research has started to examine the underlying basis of self-regulation guided by neurofeedback. Several studies have demonstrated vast activation beyond the neurofeedback target region during neurofeedback (Johnston et al., 2011; Marchesotti et al., 2017) and a recent metaanalysis has demonstrated that an extensive network of regions is shared between different mental tasks when self-regulation is guided by neurofeedback (Emmert et al., 2016). Multiple theoretical frameworks have been proposed to interpret the functional roles of involved regions (see Sitaram et al. (2017) for an overview) but extensive research on how different nodes of the network interact during neurofeedback is still pending. While previous studies have mainly focussed on understanding brain activation during neurofeedback, considerably less is known on how neurofeedback alters brain connectivity especially using the high spatial resolution of $\mathrm{fMRI}$ and its ability to observe connectivity alterations to subcortical regions. It is not yet understood how during neurofeedback task-specific networks (e.g. motor circuits recruited during motor-imagery neurofeedback) interact with networks recruited for cognitive control and feedback processing, and even less is known about how these networks are modulated through neurofeedback interventions over time.

Observing neurofeedback-induced changes on the network level is crucial from at least two perspectives: First, research on the neural underpinnings and effects of neurofeedback could benefit strongly from a network perspective on selfregulation during neurofeedback, as frequently neural working mechanisms are only observable on the level of connectivity but not prominent in analysis of regional activation (Gerchen \& Kirsch, 2017). When examining functional plasticity in the brain, connectivity analysis could also provide fundamental insights into 
training induced changes: Recent studies on learning have shown, that acquisition of novel skills lead to reorganization of connectivity profiles (Büchel, Coull, \& Friston, 1999; Daw, Niv, \& Dayan, 2005). Second, several contemporary real-time functional magnetic resonance imaging (rtfMRI) neurofeedback approaches provide participants with neurofeedback information based on connectivity between brain regions instead of mean activation within a target region (Haller et al., 2013; Kim, Yoo, Tegethoff, Meinlschmidt, \& Lee, 2015; Liew et al., 2016; Megumi, Yamashita, Kawato, \& Imamizu, 2015; Yamashita, Hayasaka, Kawato, \& Imamizu, 2017). Such connectivity neurofeedback approaches could in the future be fine-tuned based on connectivity pattern of networks involved.

Notably, relatively little research has been performed with regard to neurofeedback-induced plasticity in brain connectivity. Previous research has focussed on analysing connectivity during neurofeedback within a certain mental task domain, e.g. during neurofeedback-guided mental speech (Rota, Handjaras, Sitaram, Birbaumer, \& Dogil, 2011), auditory processing (Haller et al., 2013) or emotion regulation (Lee et al., 2011). Although previous research has shown, that different neurofeedback tasks are associated with activation in the same network (Emmert et al., 2016), it is an open issue how this network interacts with regions specifically involved in a certain mental tasks, which connectivity pattern underlie these interactions and how the emerging connectivity pattern changes over time. When examining such alterations in functional networks thoroughly, it has to be considered that interregional co-activations occur dynamically across different timescales (Sporns, 2011). Several connectivity approaches take this temporal complexity of brain activation into account, notably dynamic causal-modelling (DCM, K. J. Friston, Harrison, and Penny (2003)) and granger causality modelling (GCM, Roebroeck, Formisano, and Goebel (2005)). While multiple approaches have 
been developed to estimate functional coupling under consideration of temporal lags between regional activation (see K. J. Friston (2011) for an overview), GCM allows to investigate connectivity of one brain region to the whole brain, i.e. without restricting analysis to an a priori defined set of regions, while still providing the possibility for differentiating between lagged and instantaneous connectivity, as far as the temporal resolution of $\mathrm{fMRI}$ allows.

Considering the restricted amount of available information on brain connectivity during neurofeedback, GCM constitutes a powerful tool for exploring the connectivity of neurofeedback target regions without biasing results a priori. By applying GCM, early research on whole-brain connectivity during neurofeedback revealed the complexity of involved processes: Through examining the effective connectivity density profile of the neurofeedback target region (in this case the anterior insula, aINS) over five neurofeedback sessions, Lee et al. (2011) showed that neurofeedback training led to an early increase in overall alNS connectivity followed by a subsequent decrease with strengthening of remaining connections. Despite this network pruning over the course of training, the alNS still showed significant effective connectivity to over 20 cortical and subcortical hubs during the last session, which were either influenced by or were influencing the alNS. Additionally, analysis on training-related changes in functional connectivity during mental speech and neurofeedback have suggested increased functional connectivity between activation in the neurofeedback target region and prefrontal/parietal areas formerly implicated in language production (Rota et al., 2011). But considering that various specialized sub-networks are suggested to be involved in self-regulation with neurofeedback (see Haller et al. (2013) for a description of the network component structure of $\mathrm{fMRI}$ signals during 
neurofeedback), more fine-grained analysis of the underlying network mechanisms is pending.

This project aims to contribute to understanding the network mechanisms of neurofeedback interventions, by first, differentiating regions involved in the specific mental task used to control the neurofeedback target from the general basis of self-regulation in neurofeedback, and second, by investigating training related changes in activation and connectivity of the neurofeedback target region as revealed by GCM. To perform this analysis we used the MRI data collected during the neurofeedback training described in chapter 2 of this thesis. Due the small sample size and low statistical power of this proof of concept study, we did not expect to detect major modulations in directional connectivity on the group level. Considering also that previous studies already described alterations in directional connectivity (Lee et al., 2011) or standard functional connectivity measures (Rota et al., 2011), a particular focus of this study was instantaneous connectivity as revealed by GCM (i.e. connectivity that represents synchronicity between regional brain activation rather than directional causal influences).

In a first neurofeedback session, half of our sample $(n=10)$ got familiarized with the rtfMRI neurofeedback environment by training to gain control over the mean blood-oxygen-level dependent signal of the supplementary motor area (SMA) using motor imagery, while the other half of the sample trained to regulate the anterior insula (aINS) using positive emotion regulation. In two subsequent neurofeedback sessions, both groups continued with alNS/positive emotion regulation. This data design enabled us to observe neural correlates of neurofeedback shared between different neurofeedback tasks (session one), while also providing the possibility to observe later changes in the neural basis of emotion-regulation neurofeedback in 
the whole sample (session two and three), when participants were already familiarized with the neurofeedback environment and could focus on increasing the effectiveness of their self-regulation skills. By providing participants with rtfMRI neurofeedback and analysing whole-brain activation and connectivity during neurofeedback, we specifically aimed at answering the following research questions:

1. Which regions are specifically recruited during motor imagery and emotion regulation, and which regions are shared between these two mental tasks?

2. Does the set of regions activated during the neurofeedback training work together as a network to support regulation of the neurofeedback target, i.e. do activated regions show functional coupling / connectivity to the neurofeedback target region?

3. How does whole-brain activation and connectivity of the neurofeedback target region change over the course of a neurofeedback training?

\section{Methods and Design}

\subsection{Participants}

Ten healthy female participants (age: Mean $=22.9$ years, SD: 4.43 years; 1 lefthanded), all students of Maastricht University with normal or corrected-to-normal vision participated in the study (see chapter 2 for details). None of the participants had participated in a neurofeedback experiment before or was experienced in 
meditation. Participants were assigned to one of two training style groups (constant task scenario $[\mathrm{CON}]$ or changing task scenario $[\mathrm{CHA}])$ using a minimization procedure for outcomes on relevant markers of cognitive function, MRI experience and emotional state (see analysis section 3.1). Before each MRI scanning session, participants gave written informed consent to participate in the study. The experimental procedure was approved by the local Ethics Committee of the Faculty of Psychology and Neuroscience at Maastricht University.

\subsection{Experimental procedure}

All participants took part in a two-hour psychometric test session before the start of the neurofeedback training. Based on results of this behavioural session, participants were assigned to one of the two training-style groups (see also chapter 2, Figure 1). After acquisition of anatomical MRI and localization procedure (details below), the CHA group started the first session with SMA neurofeedback to achieve three target levels (low [20\% of the individual maxPSC], medium [ $50 \%$ of the individual maxPSC] or high [ $80 \%$ of the individual maxPSC]) using motor imagery and finished the first session with a single run of neurofeedback guided upregulation of the aINS. In the subsequent two sessions, the CHA group started with a single run of motor-imagery-guided SMA neurofeedback aimed at reaching the target levels and continued with neurofeedback-regulation to target levels using positive emotion regulation. After undergoing the same acquisition procedure of anatomical MRI and localization procedure, the CON group performed three sessions of alNS neurofeedback-regulation to target levels using positive emotion regulation across all neurofeedback runs. All neurofeedback sessions of a participant were scheduled in subsequent calendar weeks. 


\subsection{Visual stimulation and instructions}

\subsubsection{Localization procedures}

aINS localizer. Pictures with positive affect and high arousal (mean normative ratings for female participants in valence 8.23 [SD 0.23], arousal mean $=4.78$ [SD $0.74]$ ) and neutral valence and average arousal (valence mean $=4.95$ [SD 0.07], arousal 3.17 [SD 0.68]) from the International Affective Pictures System (IAPS, Lang, Bradley, and Cuthbert (1997)) were presented in randomized fashion in blocks of four pictures ( $1.5 \mathrm{~s}$ per picture, 6 sec per picture block, 12 picture blocks per valence category), alternating with $12 \mathrm{~s}$ fixation periods. The first fixation period had a duration of $16 \mathrm{~s}$ (resulting in $448 \mathrm{~s}$ in total). Participants were instructed to observe the pictures and emerging emotions. This localization paradigm has previously been shown to localize regions involved in positive affect that can be up-regulated via generation of positive emotions guided by neurofeedback and the right anterior insula was most commonly activated across participants (Johnston et al., 2011).

SMA localizer. To determine the SMA involved in motor imagery, participants were presented with a white fixation cross during rest, which turned red when participants were asked to imagine performing a movement as vivid as possible. Rest blocks (16 s) alternated with motor-imagery blocks (20 s) until participants had performed twelve motor-imagery blocks. The first rest period had a duration of 20 $\mathrm{s}$ (resulting in $452 \mathrm{~s}$ in total).

During neurofeedback runs, rest blocks (20 s) alternated with neurofeedback blocks (30 s) until participants had performed nine neurofeedback blocks, the first rest block had a duration of 90 seconds to accommodate participants to the scanning situation (resulting in $540 \mathrm{~s}$ in total). During the whole neurofeedback run, a thermometer-like display was displayed (Figure 2), consisting of ten equally sized 
segments. During rest periods, only the thermometer (white on black background) was visible and participants were instructed not to think of anything particular. During neurofeedback trials, a certain segment of the thermometer was marked red and participants were instructed to increase the appearing neurofeedback signal (depicted by segments filled in grey) and stabilize it at the height of the target level. Apart from the last run of the first session for the CHA group (where only the highest segment, [10], was marked red), the selected segments were 2, 5 and 8, alternating in random order.

\subsubsection{Self-regulation instructions}

In the psychometric session, participants in both groups were informed that they would train to achieve control over brain activation within a region of their brain involved in emotion regulation, and were briefly introduced to the target-level design. Instructions for both groups differed only with regard to task instructions during the actual neurofeedback sessions, where the CHA group was additionally provided with instructions for SMA self-regulation through motor imagery and neurofeedback.

For regulating the aINS neurofeedback signal, participants were provided with the information, that the neurofeedback signal originated from a brain region involved in positive emotions. It was suggested to participants to increase the signal by imagining or remembering positive events but that they were free to explore and identify their personal strategy, which was most effective to reach the different target levels. For regulating the SMA-neurofeedback signal, participants were provided with the information that the neurofeedback signal originated from a brain region involved in initiating movements. It was suggested to participants to increase the signal by imagining a movement while focusing on the somatic- 
experience accompanied by this movement, but that they were free to explore and identify their personal strategy, which was most effective to reach the target level. To down-regulate the neurofeedback signal across both types of training, participants were instructed to vary the intensity of their mental operation or to focus on something task-irrelevant.

\subsection{Data acquisition}

All MR images were recorded using a 3-T Siemens Magnetom Prisma MRI system (Siemens, Erlangen, Germany) with a 64-channel receiver head coil.

\section{Structural data acquisition}

All participants underwent a T1-weighted anatomical scan using a threedimensional (3D) magnetization prepared rapid-acquisition gradient-echo (MPRAGE) sequence (192 slices, slice thickness $=1 \mathrm{~mm}$, no gap, $\mathrm{TR}=2250 \mathrm{~ms}, \mathrm{TE}=$ $2.21 \mathrm{~ms}, \mathrm{FA}=9, \mathrm{FOV}=256 \times 256 \mathrm{~mm}^{2}$, matrix size $=256 \times 256$ ) in every MRI session.

\section{Functional data acquisition}

Functional images were acquired with a T2*-weighted echo-planar imaging (EPI) sequence. Except for the number of acquisitions (aINS localizer: 448 volumes; SMA localizer: 452 volumes; neurofeedback runs: 540 volumes) identical scanning parameters were used for all functional measurements that were optimized with regard to sampling frequency of $1000 \mathrm{~ms}$, which resulted in a higher temporal resolution compared to standard EPI sequences, thereby supporting specificity of connectivity analysis (48 slices, slice thickness $=2 \mathrm{~mm}, 1 \mathrm{~mm}$ gap, $\mathrm{TR}=1000 \mathrm{~ms}$, TE $=30 \mathrm{~ms}, \mathrm{FA}=50, \mathrm{FOV}=210 \times 210 \mathrm{~mm}^{2}$, matrix size $=70 \times 70$, multi-band 
acceleration factor $=3$, slice encoding direction $=$ anterior to posterior). Regarding acquisition of non-MRI data, see chapter 2 .

\section{Analysis}

\subsection{Matching}

Participants were assigned to one of two training style groups using a minimization procedure (sequential balancing, Borm, Hoogendoorn, den Heijer, and Zielhuis (2005)) with the factors previous participation in MRI experiments, estimated IQ (weighted sum of vocabulary and matrix reasoning of WAIS-IV, Pearson Education, Inc., San Antonio, TX), positive trait affect (Positive and Negative Affect Schedule Trait version (PANAS-Trait), Watson, Clark, and Tellegen (1988), positive scale), motivation to participate in the training (Freund, Kuhn, \& Holling, 2011), see chapter 2 for details.

\subsection{Neurofeedback preparation and generation}

\subsubsection{Analysis of anatomical data}

Analysis of anatomical data was performed in Brainvoyager QX Version 2.8 Anatomical data sets were corrected for spatial intensity inhomogeneity. For all participants, the anatomical data set from the first session was transferred into ACPC space and the anatomical data set from the second and third session was automatically aligned to the ACPC version of the first data set. For post-hoc analysis, anatomical data-sets were accordingly transferred into Talairach space (Talairach \& Tournoux, 1988). 


\subsubsection{Creation of neurofeedback target regions}

Neurofeedback target regions were defined in Turbo-Brain Voyager (V2.8, Brain Innovation B.V., Maastricht, the Netherlands) based on activation during the localizer runs (see Figure SII of chapter 2 for a visualization of the procedure): During the first neurofeedback session, a general linear model (GLM) was calculated on the pre-processed (motion corrected, linear trend removed and aligned to anatomical scans in ACPS space) functional data obtained during the localizer runs. From the corresponding contrast (positive pictures vs. rest [aINS localizer] and motor imagery vs. rest [SMA localizer], functional voxels were selected based on the maximal F-values located in the anatomically defined areas in which the specific regions of interest were defined (aINS for emotion regulation and SMA for motor imagery). Around the peak voxel of an activation cluster all touching voxels in the same functional slice were selected ( $3 * 3$ voxel in one slice), as well as the corresponding voxels in the slices below and above the central-voxel (resulting in a square shaped $\mathrm{ROI}: 3 * 3 * 3$ voxel in total). The peak voxel was interpolated to $1 * 1 * 1$ voxel and saved in ACPC space (this was done in order to guide ROI selection during subsequent sessions).

In the second and third session, a short resting-state localizer scan was performed (10 volumes) to determine the position of functional slices. Functional data was aligned to the T1-weighted images in of the respective session, which was aligned to the anatomical image of session one in ACPC space. Subsequently, the region of interest was created by selecting $3 * 3 * 3$ voxel in direct proximity to the central voxel of the first session's ROI, that corresponded to the respective anatomically defined areas of each neurofeedback condition (SMA or aINS). This approach supported the selection of corresponding functional voxels despite slight differences in slice positioning across sessions. Furthermore, this allowed to create regions of interest 
of the same size in 2D functional space, despite several interpolations between 2D and 3D space with differing voxel sizes (3-mm isovoxel for functional data, $1 \mathrm{~mm}$ isovoxel for T1-weighted scans); slight differences in sizes remained due to differences in slice orientation and manual selection errors (see chapter 2 for a detailed description of the ROls). For further offline-analysis, the 2D functional ROIs where interpolated to $3 \mathrm{D}$ volumes of interest (VOIs) and transferred into ACPC space.

\subsubsection{Generation of neurofeedback information}

The neurofeedback signal was calculated with following the general procedure previously validated for neurofeedback target-level designs by Sorger, Kamp, Weiskopf, Peters, and Goebel (2016): In short, the percent signal change (PSC) of the current pre-processed volume of the consecutive runs was calculated as mean activation of all voxels within the particular ROI, with respect to a local baseline. For the current study, the percent signal change (PSC) of a given volume was calculated as the temporarily smoothed (by a factor of 6; i.e., the mean of the current and the five previous time-points) to stabilize the feedback signal acquired with the relatively high TR of $1000 \mathrm{~ms}$. The average activation during the preceding rest block constituted the baseline for the consecutive neurofeedback block.

The PSC was then normalized by a maximum PSC (maxPSC), which was defined as follows: For emotion regulation, the maxPSC was set to $1 \%$ for all participants, to verify comparability between groups. For SMA regulation, the maxPSC was determined based on the mean percent signal change in the selected voxels of the localizer run (mean $=1.4 \%, \mathrm{SD}=0.42 \%$; see chapter 2 ). The PSC at each TR was transformed into a feedback value on a scale between 0 and 10 , where 10 
corresponded to maxPSC and each segment to $10 \%$ of the maxPSC in relation to the previous baseline period, so that the target levels 3, 5, 8 corresponded to $30 \%, 50 \%$ and $80 \%$ of the maxPSC. In the thermometer display provided to participants, values below zero and above 10 were displayed as " 0 " and " 10 ", respectively.

\subsection{Post-hoc analysis of (f)MRI data}

All analysis was performed using BrainVoyager QX (v2.8, Brain Innovation, Maastricht, the Netherlands).

\subsection{1 (f)MRI Pre-Processing}

Anatomical data sets were corrected for spatial intensity inhomogeneity. For all participants, the anatomical data set from the first session was transferred into ACPC space and the anatomical data sets from the second and third sessions were automatically aligned to the ACPC version of the first data set. All data sets were spatially normalized by Talairach transformation. All functional data sets underwent slice scan-time correction, linear trend removal and temporal high-pass filtering (two cycles per time course). Three-dimensional (3D) head-motion detection and correction was applied by spatially aligning all functional volumes of a session to the first functional volume of the first run within that session. Finally, all functional runs were spatially normalized to Talairach space and interpolated to a 3-mm ${ }^{3}$ voxel resolution. For whole-brain and masked analysis, functional data was smoothed in 3D with a 6-mm FWHM Gaussian kernel. 


\subsubsection{Activation within and across neurofeedback modalities}

To reveal activation specific to a certain neurofeedback task, as well as activation shared across different neurofeedback tasks, a twofold analysis was performed on the data of session one, in which both groups performed different neurofeedback tasks (CON: emotion-regulation with alNS neurofeedback; CHA: motor-imagery with SMA neurofeedback):

First, to take into account the low sample size within each neurofeedback modality $(n=5)$, at first an exploratory analysis was performed: A whole-brain fixed-effects general-linear model (FFX GLM) was carried out within each neurofeedback modality (emotion regulation / aINS neurofeedback and motor imagery / SMA neurofeedback), including the factors neurofeedback target levels (low: 20\%, medium: $50 \%$, high: $80 \%$ of the individual maxPSC), as well as six motion parameters as confounding predictors. Resulting maps were Bonferroni corrected at a threshold of $p=0.005$. To identify regions that were activated in the aINS group and the SMA group, an intersection analysis was performed on the multiplecomparison corrected t-maps: Significant activation clusters were transformed into 3D volumetric representations in Talairach space, using a cluster threshold of 540 anatomical voxel, and intersections between the 3D volumes of both groups were defined (logical AND). This approach constituted a first exploratory investigation of the overlap that aimed to determine the detectability of the neurofeedback network in small samples $(n=5)$ and its spatial consistency across neurofeedback modalities.

Second, to apply a stricter statistical criterion, a random-effects-analysis GLM (RFX) was calculated across all participants, including the factor target levels (low, medium, high) and neurofeedback modality (emotion and motor imagery), as well 
as six motion parameters as confounding predictors. To prevent type two errors due to the sample size, analysis was restricted to voxels within $15 \mathrm{~mm}$ radii around cortical and $20 \mathrm{~mm}$ radii around two subcortical meta-analytic peak voxels centred in the thalamus (with the resulting mask also including the basal ganglia), that marked activation increases across several neurofeedback studies compared to rest (Emmert et al., 2016). Additionally, overlap between feedback modalities with this network was specified by performing a conjunction analysis of RFX activation of the two neurofeedback conditions.

\subsubsection{Connectivity within and across neurofeedback modalities}

To investigate which regions synchronize activation with the target region during neurofeedback (i.e. not only showing generally increased activation during neurofeedback periods), a twofold analysis was performed:

First, for each participant, Granger causality maps (GCMs) were computed from signal time courses of the first session between voxels in the neurofeedback target region and the rest of the brain in the scanned volume. The average time course within modulation periods of individual neurofeedback regions was taken as a reference to estimate the instantaneous non-directed correlation and the directed correlation by considering it as a potential target of influence from voxels from the rest of the brain, as well as a potential source (for an overview of the procedure see Goebel, Roebroeck, Kim, and Formisano (2003), Roebroeck et al. (2005)). Subsequently, significant instantaneous and directed connectivity of the target region was identified using a t-test against 0 on all individual connectivity maps (cluster corrected at $p=0.001$ ) within each feedback modality as well as across all participants. This step was performed separately for instantaneous and directed connectivity maps. This analysis resulted in six group maps (emotion, motor 
imagery, respectively for directional and instantaneous connectivity). The created maps showed voxels that underwent functional coupling with the neurofeedback target region while discarding major directional influences (instantaneous connectivity) or directional connectivity. Connectivity maps of individual participants were inspected for major interference of global variations in signal intensity.

Second, to determine whether regions showing the previously described activation increases and decreases during neurofeedback also functionally interact with the neurofeedback target region, intersections between connectivity and activation cluster were investigated: Cluster with significant connectivity to the target region on the group level were transferred into volumetric representations and their intersections with volumetric representations of significant FFX activation clusters shared between both training groups was determined (logical AND; see Table 1, "shared cluster").

\subsubsection{Activation during emotion regulation over time}

To reveal activation constant and changing over time during emotion-regulation neurofeedback, a twofold analysis was performed:

First, to reveal activation across the whole brain constant across and different between sessions, while taking account the low sample size (per session and group: $n=5$ ), at first an exploratory analysis was performed: Within each learning style group (constant $[\mathrm{CON}]$ and changing $[\mathrm{CHA}]$ ) and full-sample emotion regulation session (session two and three) one FFX GLM analysis was performed using the factors neurofeedback target-level (low, medium, high) and six motion parameters as confounding predictors. Effects were modelled using a boxcar function, which 
was convolved with the Two-Gamma hemodynamic impulse function (K. Friston et al., 1998). This resulted in four activation maps for [neurofeedback > baseline], one per group and session. The maps were each Bonferroni corrected at a threshold of $p=0.005$. To identify regional activation present within each group and session, an exploratory intersection analysis was performed on the multiple-comparison corrected t-maps: Significant activation clusters within each session and group were transformed into 3D volumetric representations in Talairach space, using a cluster threshold of 540 anatomical voxel, and intersections between the 3D volumes of both groups were defined (logical AND). This approach constituted a first exploratory investigation of the overlap that aimed to determine the detectability of the neurofeedback network in small samples $(n=5)$ and its spatial consistency across training time-points and training styles.

Second, a stricter statistical criterion was applied for analysing activation pattern during session two and three: A RFX GLM analysis was carried out across all participants, including the factors neurofeedback (target-level low, medium, high) and time (session two and three) and six motion parameters as confounding predictors. Effects were modelled using a boxcar function, which was convolved with the Two-Gamma hemodynamic impulse function (K. Friston et al., 1998). To prevent type two errors analysis was restricted to voxels within $15 \mathrm{~mm}$ radii around cortical (anterior cingulate cortex $[A C C]$, ventrolateral and dorsolateral PFC [VIPFC / dIPFC], temporo-parietal cortex, parietal cortex) and $20 \mathrm{~mm}$ radii around and two subcortical (centred in the thalamus, with the resulting mask also including the basal ganglia) meta-analytic peak voxels, that marked activation increases across several neurofeedback studies (Emmert et al., 2016). Additionally, overlap across feedback modalities with this network on the group level was further investigated by subsequently performing a conjunction analysis of Session three and Session 
two. Activation changes over time were investigated by contrasting Session three > Session two.

\subsubsection{Connectivity over time}

To reveal whether whole-brain connectivity of the neurofeedback target region changes over time, a twofold analysis was performed:

First, to determine statistically significant connectivity profiles within each full emotion regulation training session (session two and three, each $n=10$ ), a t-test against 0 on all individual GCM connectivity maps within the given session was performed (separately for instantaneous and directed connectivity maps). Subsequently, connectivity maps of individual participants were inspected for major influences of variations in global signal intensity. While global variations in signal intensity have been shown to reduce the specificity of connectivity estimates (Murphy \& Fox, 2017), overcorrection of global effects also carries the risk of inducing false negatives into analysis (i.e. correcting true effects, see Weissenbacher et al. (2009)). Due to the risk of corrupting real condition effects, only individual participants' connectivity maps showing target region connectivity to all voxels of the brain at an participant threshold of $\operatorname{FDR}(q)<0.01$ were reprocessed with additional adjustment for mean intensity (MIA) of functional datasets. To understand whether subsequent results were influenced by unspecific effects of the MIA, the respective participants' data was additionally adjusted for mean intensity at the other timepoint as well and compared with uncorrected results.

Second, after verification of significant group results (only present for instantaneous connectivity) an exploratory analysis was conducted to describe the 
instantaneous connectivity pattern within groups as well as within sessions, to provide more nuanced insights into potential mechanisms underlying the results of the main statistical analysis: After estimating instantaneous connectivity maps for all participants, sessions and groups in relation to neurofeedback target region as described before (resulting in 30 connectivity maps in total), within each time-point and group shared connectivity pattern were revealed using a t-test against 0 with a threshold of $p=0.005$ and $p=0.1$ to provide a descriptive overview on the small sample connectivity pattern $(n=5)$.

\section{Results}

\subsection{Activation within and across neurofeedback modalities}

To reveal activation specific to a certain neurofeedback task, as well as activation shared across different neurofeedback tasks, a twofold analysis was performed on the data of session one, in which both groups performed different neurofeedback tasks (CON: emotion-regulation with alNS neurofeedback; $\mathrm{CHA}$ : motor-imagery with SMA neurofeedback):

First, comparing whole-brain FFX GLM results for neurofeedback > rest within each task modality (Figure 1, A Table 1) revealed activation of both neurofeedback tasks in the general locations consistent with previous meta-analytical evidence on a particular network activated during neurofeedback (Emmert et al., 2016), specifically in prefrontal areas (including the bilateral aINS, vIPFC, dIPFC, ACC and SMA), lateral parietal lobe (LPL; including the intraparietal sulcus, IPS) and lateral 
occipital cortex (LOC) and the left anterior striatum, and additionally deactivation in the posterior insula (pINS).

\begin{tabular}{|c|c|c|c|c|c|c|}
\hline Region & Center & $o f$ & & Extension & & \\
\hline Shared Cluster & $\mathbf{x}$ & $\mathbf{y}$ & $\mathbf{z}$ & Voxel & Emotion & Motor \\
\hline Lateral Parietal Lobe R & 38.59 & -45.15 & 40.03 & 6648 & 7.07 & 7.68 \\
\hline Lateral PFC / aINS R & 43.77 & 7.14 & 27.15 & 10354 & 8.08 & 7.81 \\
\hline Medial Temporal Gyrus R & 48.97 & -53.45 & -8.85 & 1603 & 6.34 & 7.75 \\
\hline LOC R & 37.62 & -86.23 & 3.46 & 816 & 6.39 & 7.61 \\
\hline SMA & -1.75 & -5.97 & 60.03 & 5706 & 9.59 & 7.80 \\
\hline Medial frontal Gyrus / ACC & 0.28 & 15.96 & 48.78 & 860 & 7.29 & 6.35 \\
\hline Anterior Striatum L & -18.88 & 1.43 & 5.34 & 231 & 6.34 & 5.96 \\
\hline Lateral Agranular Frontal Cortex R & -40.85 & -1.68 & 37.04 & 12057 & 8.61 & 8.41 \\
\hline aINS L & -29.85 & 19.58 & 9.68 & 1952 & 8.80 & 7.64 \\
\hline dlPFC L & -39.49 & 33.26 & 29.76 & 1957 & 7.75 & 7.41 \\
\hline Lateral Parietal Lobe L & -37.06 & -49.51 & 43.62 & 10109 & 7.83 & 7.53 \\
\hline pINS & 37.31 & -18.63 & 18.25 & 432 & -6.47 & -6.19 \\
\hline Cerebellum & 9.16 & -55.22 & -24.8 & 6264 & 7.44 & 7.00 \\
\hline
\end{tabular}

Emotion

Emotion
\begin{tabular}{|l|c|c|c|c|c|c|}
\hline Medial Temporal Gyrus R & 60.05 & -14.85 & -2 & 1485 & -6.58 & -1.47 \\
\hline dlPFC R & 28.13 & 32.25 & 30.63 & 648 & 5.96 & 2.78 \\
\hline Paracentral Lobe / Precuneus & 6.81 & -38.85 & 69.31 & 702 & -5.78 & -1.03 \\
\hline Posterior Cingulate / Precuneus & -2.17 & -48.17 & 29.09 & 2538 & -6.21 & -2.48 \\
\hline Left occipital Lobe & -10.05 & -87.21 & 5.42 & 9315 & -6.62 & 1.26 \\
\hline Frontopolar Cortex & -25 & 44.33 & -4.67 & 729 & 6.20 & 1.47 \\
\hline Cerebellum & -28.11 & -82.78 & -32.22 & 729 & -5.75 & 4.13 \\
\hline Anterior Temporal Lobe / aINS & -54.53 & -6.82 & -0.41 & 4428 & -6.50 & -2.23 \\
\hline Medial Occipital Lobe R & 11.49 & -91.2 & -7.14 & 1593 & -6.11 & 0.86 \\
\hline LOC / Posterior Temporal Lobe L & -48.27 & -50.19 & 2.23 & 4158 & 6.54 & 3.52 \\
\hline
\end{tabular}

Motor Imagery

\begin{tabular}{|l|c|c|c|c|c|c|}
\hline Postcentral Gyrus & 29.68 & -40.11 & 67.75 & 756 & -3.55 & -6.27 \\
\hline Anterior striatum R & 14.16 & 3.59 & 11.03 & 864 & 4.52 & 5.84 \\
\hline Brainstem & -0.23 & -22.77 & -1.77 & 1053 & 3.78 \\
\hline Inferior temporal Gyrus & -56.43 & -30.43 & -20 & 567 & 2.67 & 6.15 \\
\hline
\end{tabular}

Table 1: Whole-brain activation during session one, FFX results. The table contains location, extension and statistical properties of significant clusters revealed by whole-brain FFX analysis (neurofeedback $>$ rest) thresholded at Bonferroni corrected $p<0.005$, including clusters with over 540 anatomical voxel (coordinates in Talairach space) for clusters showing overlap between both neurofeedback modalities, clusters only active during emotion neurofeedback and clusters only active during motor-imagery neurofeedback. Remarks: $L$, left; $R$, right, otherwise bilateral. 

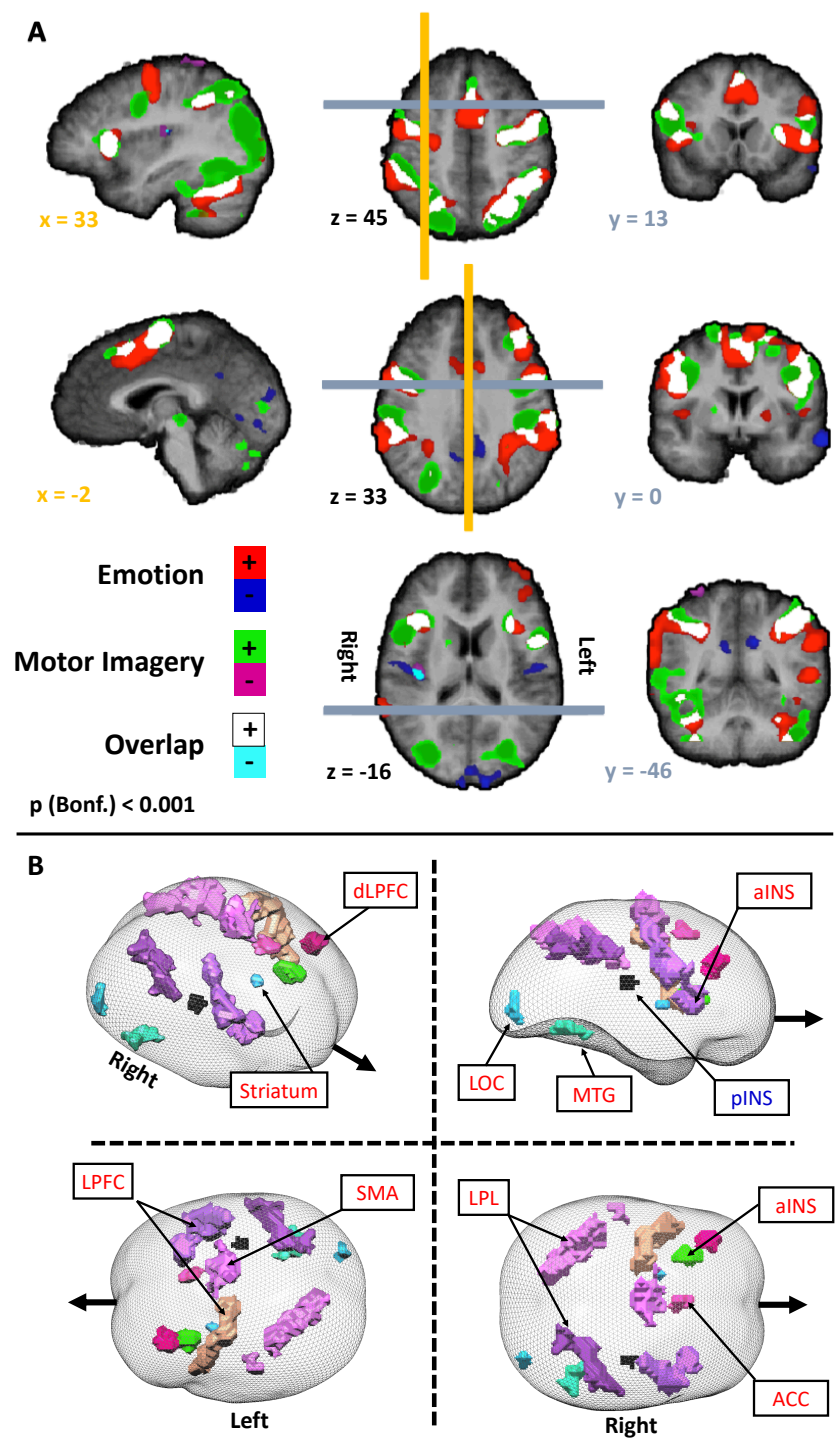

Figure 1: Whole-brain FFX activation results of emotion and motor-imagery neurofeedback. The figure displays whole-brain activation during motor imagery with SMA neurofeedback and emotion regulation with alNS neurofeedback during session one compared to rest. A. Groups showed different activation pattern in mental task-specific regions (Emotion regulation was associated increases in anterior frontal areas, as well as more pronounced deactivation of the DMN and the occipital lobe, while motor imagery was associated by modulations in lateral parietal areas and increased brainstem activation). Both neurofeedback modalities showed (de-) activation in cortical and subcortical clusters associated with neurofeedback guided self-regulation (turquoise/white). Results are 
overlaid on the average group anatomy. B. Three dimensional reconstructions of main overlap clusters (over 300 voxel size) closely resembled recent meta-analytical findings on neural correlates of neurofeedback with additional deactivation in the pINS (black), see Table 1 for details. Remark: Colour variations of clusters in B for visibility.

During emotion regulation also dIPFC and frontopolar activation was observable as well as deactivation in the occipital cortex, left posterior insula (pINS) cortices and the precuneus, a major hub of the default-mode network (DMN), while during motor imagery distributed activation and deactivation in occipito-parietal areas could be observed as well as increased brainstem, inferior temporal gyrus and right anterior striatum activation. Intersection analysis (Figure 1, B; , Table 1) revealed overlapping activation of both neurofeedback task modalities in the bilateral lateral PFC (including aINS), lateral parietal cortex, SMA, ACC/medial frontal gyrus (MFG), cerebellum, left anterior striatum, right lateral occipital cortex and deactivation in the right pINS.

Second, RFX GLM analysis within the neurofeedback network (Emmert et al., 2016) supported group overlap results, revealing significantly increased activation in main hubs of the network when pooled across both task modalities (Figure 2), for details see Table 2. A conjunction analysis of within group RFX results did not result in significant conjunction clusters, a contrast analysis of emotion regulation > motor imagery did not reveal significant differences between task modalities in the neurofeedback network. 


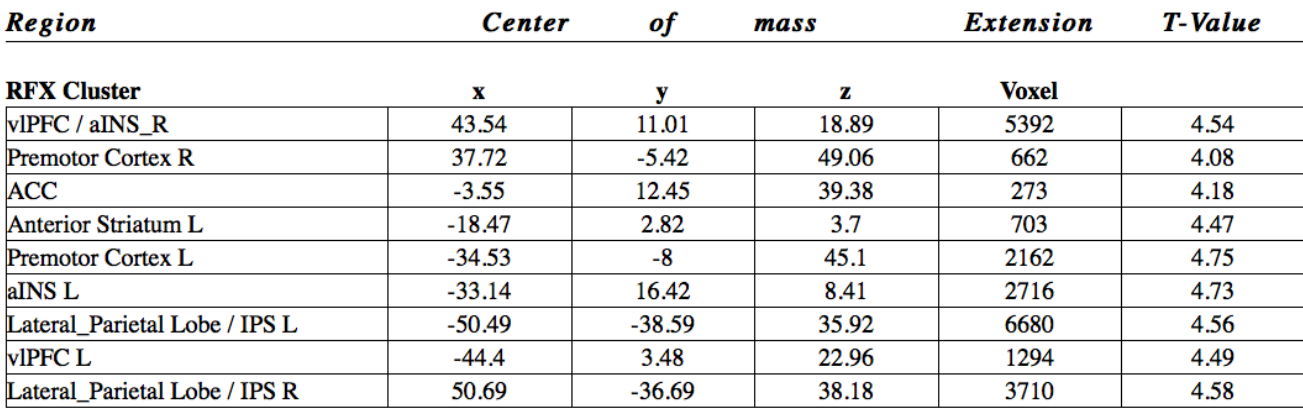

Table 2: Brain activation in the neurofeedback network across neurofeedback modalities during session one, RFX results. The table contains location, extension and statistical properties of significant clusters revealed by RFX analysis (neurofeedback > rest) in $15 \mathrm{~mm}$ proximity to cortical activation peaks and $20 \mathrm{~mm}$ of the subcortical activation peak of the neurofeedback network described by Emmert et al. (2016). Results were FDR corrected with $q<0.05$ (coordinates in Talairach space). Remarks: L, left; R, right, otherwise bilateral.
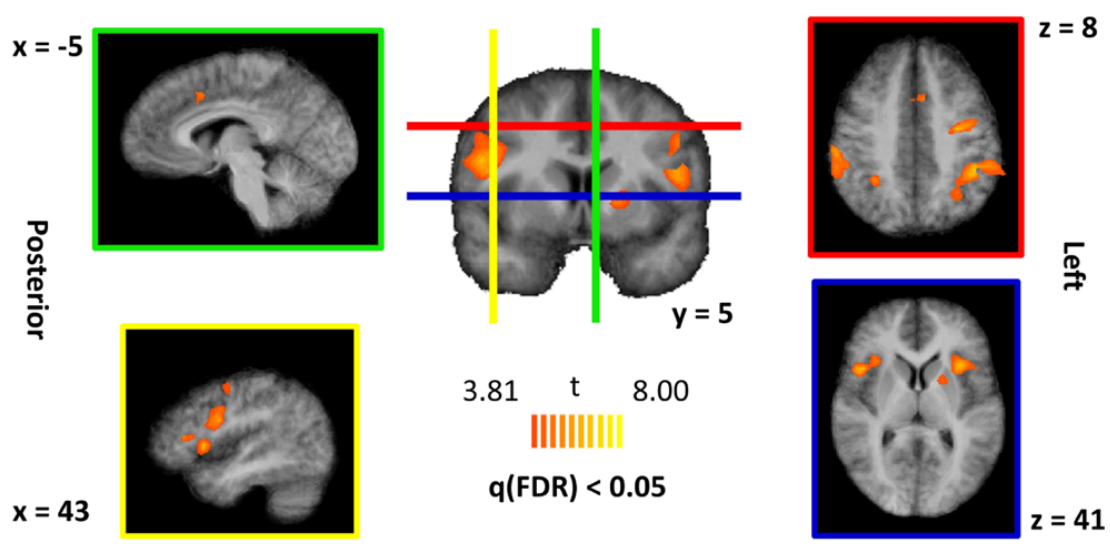

Figure 2: Neurofeedback network RFX results across emotion and motor-imagery neurofeedback. The figures displays RFX results across motor imagery/SMA neurofeedback and emotion regulation/ alNS neurofeedback in session one compared to rest, for areas previously described as being reliably activated during neurofeedback by Emmert et al. (2016) overlaid on the average group anatomy. Across both neurofeedback modalities, significant activation was detected in the vIPFC, alNS, striatum, SMA/premotor cortex and lateral PL, see Table 2 for details. 


\subsection{Connectivity within and across neurofeedback modalities}

To investigate which regions synchronize activation with the target region during neurofeedback (i.e. not only showing generally increased activation during neurofeedback periods), a twofold analysis was performed:

First, t-tests against zero within neurofeedback modalities revealed no significant directed connectivity but significant instantaneous connectivity between an occipital cluster and the neurofeedback target region (SMA) during motor imagery (Figure 3, A; Table 3), but no significant connectivity during emotion regulation (for the aINS). A distributed network of regions with significant connectivity could be observed when pooling over both neurofeedback modalities, including the precuneus, cerebellum, occipital cortex, ACC, temporoparietal junction (TPJ), vIPFC and dIPFC (Figure 3, B; Table 3).

Second, intersection analysis between connectivity to target regions across groups and activation increases in the neurofeedback network revealed that the majority of regions with increased activation also show increased connectivity to the respective neurofeedback target region: Increased activation and connectivity overlapped in the ACC, IFG, lateral prefrontal cortex, cerebellum and occipital lobe and deactivation with connectivity to the pINS, the lateral parietal lobe and the pINS (Figure 3, Table 4). 

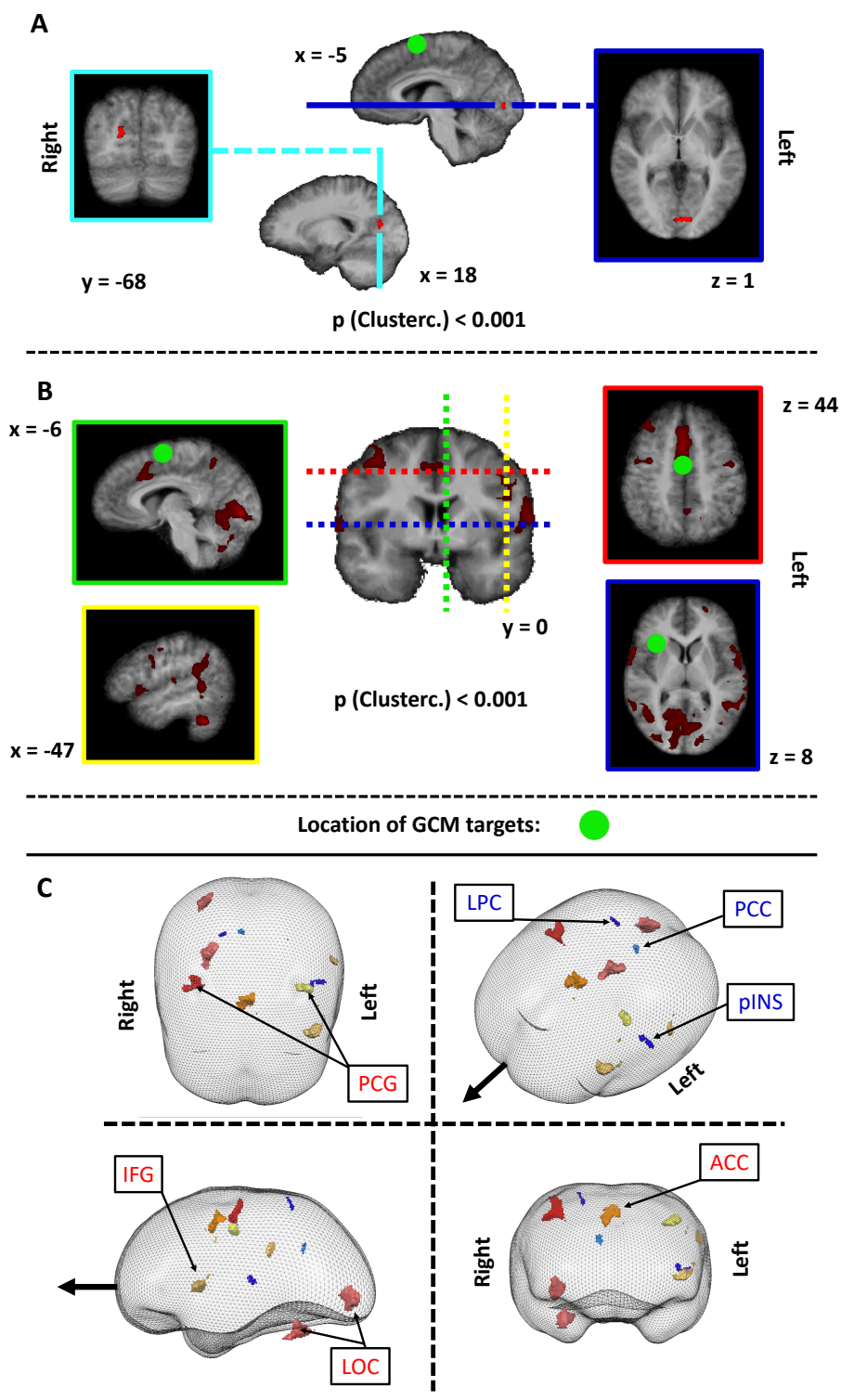

Figure 3: Instantaneous connectivity of the neurofeedback target regions during emotion and motor-imagery neurofeedback. The figure displays instantaneous connectivity of the respective neurofeedback target region during motor imagery (SMA, green) and emotion regulation (right alNS, purple) guided by neurofeedback overlaid on the average group anatomy. A. Connectivity of SMA during motor-imagery neurofeedback to cuneus and right lateral occipital cortex (no significant connectivity of the aINS during emotion regulation), see Table 3. B. Connectivity pooled across both neurofeedback modalities revealed 
significant connectivity across both respective target regions (SMA or aINS) to a network of cortical and subcortical clusters of the neurofeedback network C. Three-dimensional reconstructions of connectivity across groups overlapped in most clusters with whole-brain FFX activation results (overlap with activation: yellow/red; Overlap with deactivation: blue), see Table 4.

\begin{tabular}{l} 
Region \\
\hline
\end{tabular}
\begin{tabular}{|c|c|c|c|c|c|} 
Motor Imagery & $\mathbf{x}$ & of & mass & Extension & T-Value \\
\hline Cuneus & 18.73 & -68.34 & 17.16 & 310 & 10.15 \\
\hline Lateral Occipital Lobe & -2.77 & -83.31 & -1.07 & 546 & 10.43 \\
\hline
\end{tabular}

\section{Emotion}

Across Groups

\begin{tabular}{|c|c|c|c|c|c|}
\hline Occipital Lobe / Cerebellum & 7.22 & -66.65 & -6.72 & 58986 & 5.31 \\
\hline vlPFC R & 57.15 & 8.96 & 6.88 & 2038 & 5.46 \\
\hline Precentral Gyrus / Premotor Cortex R & 37.86 & -3.86 & 51.29 & 2002 & 5.27 \\
\hline Precuneus & 0.28 & -53.9 & 48.27 & 1380 & 5.08 \\
\hline $\mathrm{ACC}$ & 0.79 & 15.02 & 42.92 & 7996 & 5.48 \\
\hline Precentral Gyrus / Premotor Cortex L & -44.35 & -2.47 & 37.05 & 1164 & 5.05 \\
\hline Lateral Parietal Lobe / vlPFC L & -54.81 & -27.5 & 16.74 & 15079 & 5.35 \\
\hline Precentral Gyrus R & 57.09 & 9.01 & 6.72 & 2006 & 5.44 \\
\hline Temporoparietal Junction $\mathrm{R}$ & 55.35 & -43.65 & 24.6 & 1059 & 5.16 \\
\hline dlPFC & 35.19 & 31.4 & 44.64 & 825 & 5.31 \\
\hline Lateral Parietal Lobe R & 18.35 & -38.85 & 57.81 & 399 & 5.15 \\
\hline Posterior Cingulate Gyrus & 11.87 & -47.52 & 33.38 & 1154 & 5.25 \\
\hline Cerebellum L & -13.72 & -27.42 & -18.9 & 621 & 5.31 \\
\hline Frontopolar Cortex L & -22.69 & 54.73 & 14.57 & 1033 & 5.13 \\
\hline Middle Occipital Gyrus L & -24.19 & -74.32 & 21.37 & 474 & 4.98 \\
\hline
\end{tabular}

Table 3: Instantaneous connectivity results for session one. The table contains location, extension and statistical properties of clusters with significant connectivity to the respective neurofeedback target region during neurofeedback periods, cluster corrected at $p<0.001$, for connectivity during motor imagery/SMA neurofeedback, emotion regulation/aINS neurofeedback and connectivity pooled across both neurofeedback modalities. Remarks: L, left; R, right, otherwise bilateral. 


\begin{tabular}{l|c|c|c|c|}
\begin{tabular}{l} 
Region \\
\hline
\end{tabular} & \multicolumn{1}{c}{ Center } & of & \multicolumn{2}{c}{ mass } \\
$\begin{array}{l}\text { Overlap with activation } \\
\text { Lateral Occipital Lobe / Cerebellum }\end{array}$ & $\mathbf{x}$ & $\mathbf{y}$ & $-11,38$ & 1417 \\
\hline Precentral Gyrus R & 32,86 & $-68,77$ & 50,37 & 476 \\
\hline ACC & 40,72 & $-5,21$ & 46,14 & 403 \\
\hline Precentral Gyrus L & 2,1 & 6,76 & 42,67 & 309 \\
\hline IFG & $-41,64$ & $-6,1$ & 10,84 & 532 \\
\hline
\end{tabular}

Overlap with deactivation

\begin{tabular}{|l|c|c|c|c|}
\hline PINS / Superior Temporal Sulcus & $-48,84$ & $-21,07$ & 10,95 & 82 \\
\hline Lateral Parietal Lobe R & 20,16 & -41 & 60,34 & 38 \\
\hline Posterior Cingulate Gyrus & 7,12 & $-51,98$ & 32,86 & 42 \\
\hline
\end{tabular}

Table 4: Overlap between whole-brain activation and connectivity of the respective neurofeedback target region during session one. The table contains location and extension of overlap between connectivity maps and whole-brain activation (FFX) shared between both neurofeedback modalities. Remarks: L, left; R, right, otherwise bilateral.

\subsection{Activation during emotion-regulation neurofeedback over time}

To reveal activation stable and changing over time during emotion regulation / aINS neurofeedback, twofold results were acquired:

First, whole-brain FFX GLM analysis carried out within each group (learning schedule either constant ( $\mathrm{CON}$ ) or changing ( $\mathrm{CHA})$ ) revealed stable (de-)activation in the neurofeedback network and emotion regulation regions across both groups and sessions (Figure 4, A; Table 5), with overlapping activation increases in the bilateral aINS, vIPFC, dIPFC, fronto-polar cortex, bilateral inferior temporal gyrus, distributed parietal areas, lateral occipital cortex (LOC) as well as shared deactivation in the DMN (vmPFC, precuneus/ posterior cingulate) and the bilateral pINS. Comparing session two and three, both groups shared extended deactivation in the OFC and the anterior default mode network (VmPFC) only during the last session. Overall, FFX maps of groups and sessions showed high consistency in 
cortical (de-)activation clusters of the neurofeedback network, but not in subcortical areas, where the anterior striatum was activated significantly only by the CHA group during both sessions, and striatal activation changed from extensive bilateral anterior striatum activation during session two to a more defined activation in the right anterior striatum during session three (Supplementary Figure SI).

Second, the RFX GLM analysis across all participants (Figure 4, B; Table 6) restricted to voxel in proximity of activation peaks of the neurofeedback network supported the involvement of a general network of regions involved not only across tasks, but also across time, with distributed activation increases across the neurofeedback network, specifically in the bilateral dIPFC, VIPFC, SMA, and deactivation in the bilateral pINS, while conjunction analysis and contrast analysis between Session three and Session two did not reveal any significant conjunction or differences. 
A
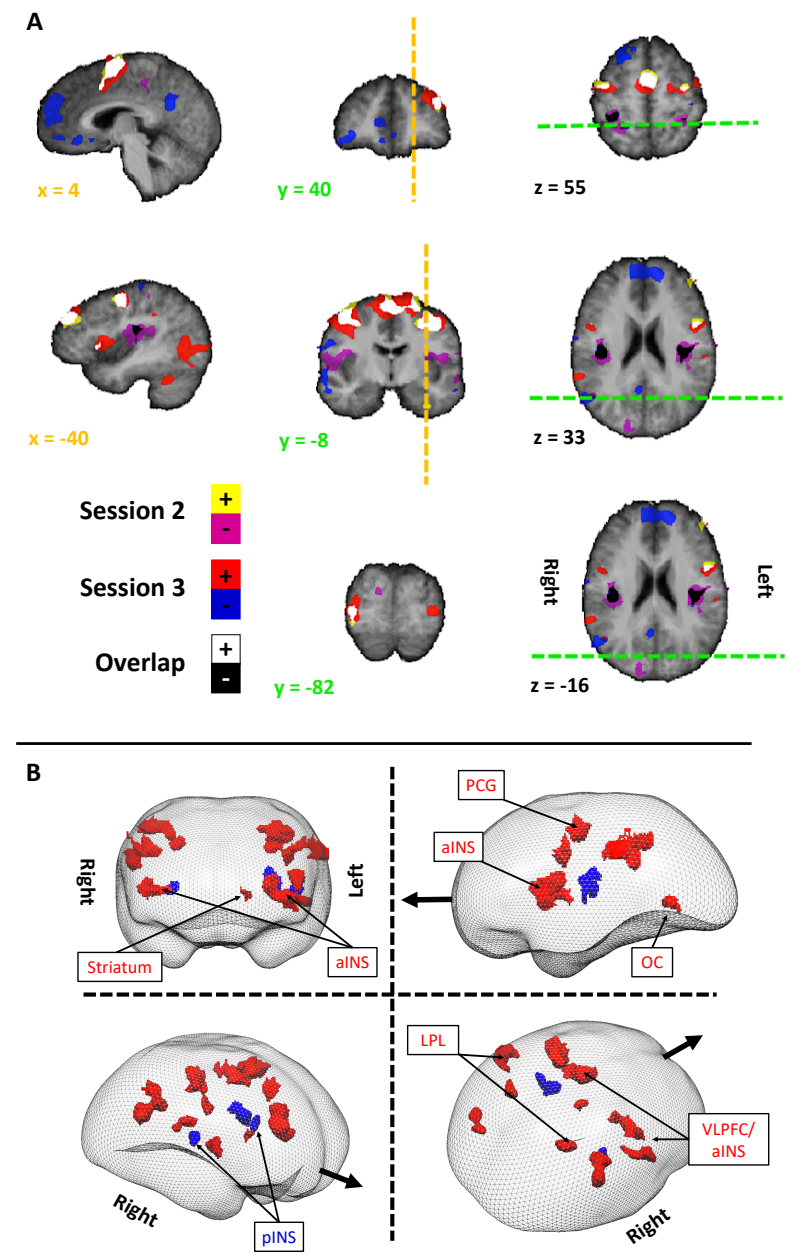

Figure 4: Effect of time on brain activation during aINS neurofeedback (session two and three). A. Activation specific to or shared between session two and three during neurofeedback compared to rest overlaid on the average group anatomy (FFX results). The figure shows overlap in significant activation clusters during alNS neurofeedback between the CON and the CHA group during session two (yellow, purple) and session three (red, blue). During both sessions, the groups shared activation in cortical clusters of the neurofeedback network (white) and deactivation in the pINS (black), but notably not in subcortical clusters. Session three was associated with more extensive deactivation in the DMN and the anterior PFC across both groups. B. Three dimensional reconstructions of activation during aINS-neurofeedback in the neurofeedback network across sessions 2 and 3 (RFX results). Activation increases in lateral prefrontal, parietal and occipital regions and deactivation in the pINS. 


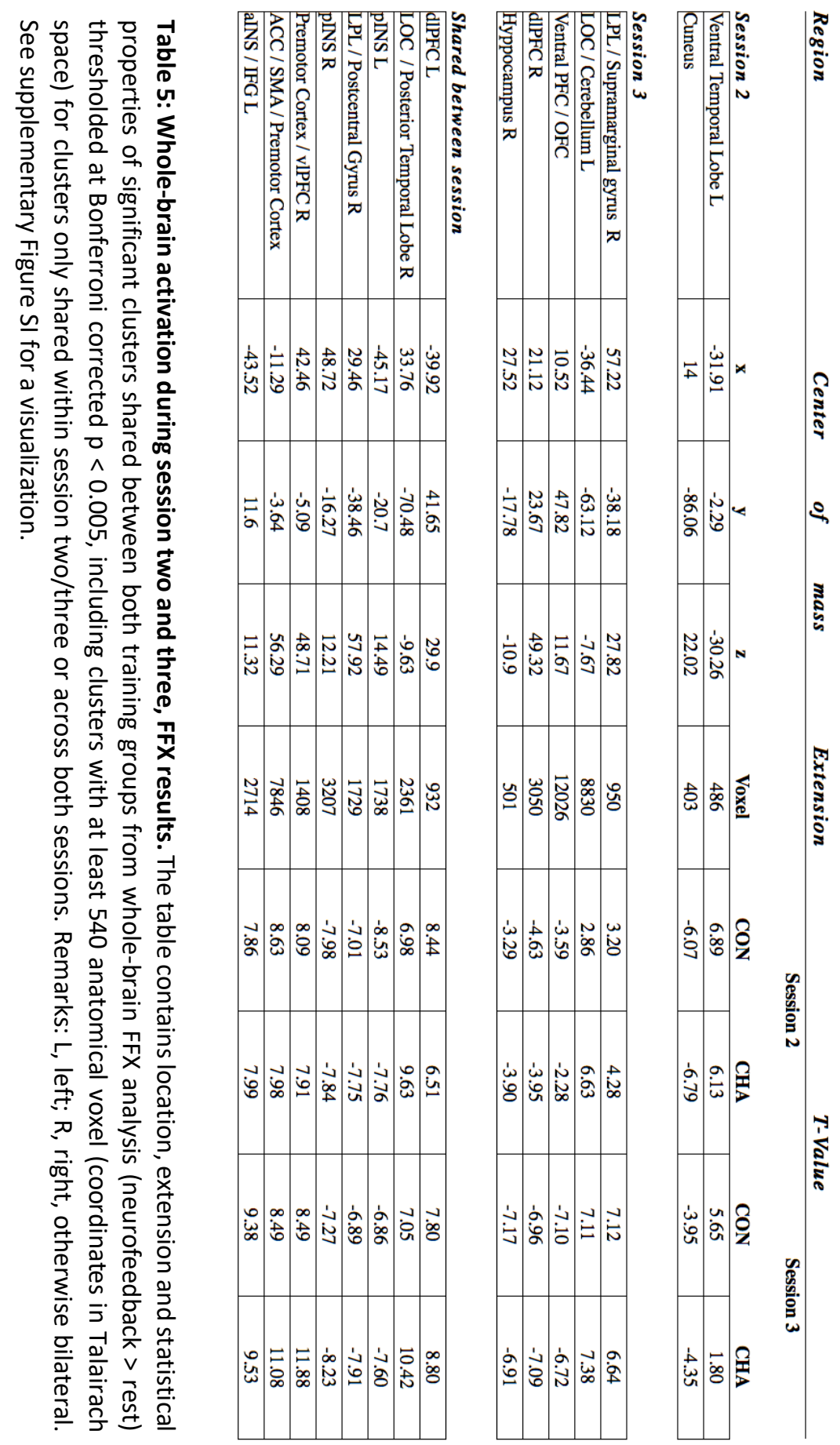




\begin{tabular}{|c|c|c|c|c|c|}
\hline Region & Center & of & & Extension & T-Value \\
\hline & $\mathbf{x}$ & $\mathbf{y}$ & $\mathbf{z}$ & Voxel & \\
\hline Precentral Gyrus R & 44.18 & -6.04 & 48.43 & 707 & 4.60 \\
\hline Striatum L & -15.84 & 0.58 & 5.63 & 118 & 4.35 \\
\hline Precentral Gyrus L & -36.17 & -6.65 & 45.65 & 1494 & 5.04 \\
\hline aINS L & -35.95 & 14.47 & 7.36 & 1757 & 4.80 \\
\hline Occipitotemporal Junction & -47.26 & -62.56 & 2.2 & 508 & 4.44 \\
\hline vlPFC/ aINS & 45.62 & 5.44 & 17.55 & 1160 & 4.60 \\
\hline Lateral Parietal Lobe $\mathrm{R}$ & 49.65 & -37.52 & 34.57 & 2065 & 5.02 \\
\hline vlpfe L & -46.42 & 5.21 & 21.58 & 1300 & 4.98 \\
\hline Lateral Parietal Lobe L & -52.62 & -35.54 & 35.77 & 1870 & 4.93 \\
\hline pINS R & 31.97 & -14.84 & 10.43 & 199 & -4.66 \\
\hline pINS L & -34.75 & -12.01 & 13.88 & 1013 & -4.85 \\
\hline
\end{tabular}

Table 6: Brain activation in the neurofeedback network during emotion-regulation neurofeedback across sessions 2 and 3, RFX results. The table contains location, extension and statistical properties of significant clusters revealed by RFX analysis (neurofeedback > rest) in $15 \mathrm{~mm}$ proximity to cortical activation peaks and $20 \mathrm{~mm}$ of the subcortical activation peak of the neurofeedback network described by Emmert et al. (2016). Results were FDR corrected with $q<0.05$ (coordinates in Talairach space). Remarks: L, left; R, right, otherwise bilateral.

\subsection{Connectivity during emotion-regulation neurofeedback over time}

To reveal whether whole-brain connectivity of the neurofeedback target region undergoes changes over time, a twofold analysis was performed:

First, analysis connectivity on the full-sample emotion regulation sessions two and three (t-test against 0; Figure 5, A; Table 7) revealed no significant directed connectivity. In session two, significant instantaneous connectivity was present to hubs of the neurofeedback network, i.e. right ACC, vIPFC, aINS, bilateral dIPFC, TPJ, LPL, and occipital cortex (OC) / cerebellum, left medial temporal gyrus (MTG), while during session three no significant instantaneous connectivity was present. Subsequent inspection of individual connectivity maps revealed that one participant time-point (P04, session three, CHA group) showed significant 
connectivity between the target region and all voxels of the brain at a threshold of $F D R(q)<0.01$ imposing a major influence on group statistics (supplementary Figure SII): After adjustment for mean intensity of this data-set, group results for instantaneous connectivity in session three showed high consistency (i.e. overlap) with connectivity results of session two: Major hubs of the neurofeedback network showed significant connectivity to the target region, i.e. right vIPFC and MTG, bilateral ACC, dIPFC, aINS, LPL, OC / cerebellum, posterior cingulate cortex (PCC), precuneus, left dLPFC, and anterior striatum. Connectivity cluster of session two and three overlapped in the right ACC, VLPFC, alNS, bilateral dIPFC, precuneus, LPL and $\mathrm{OL} /$ cerebellum. To test whether significant connectivity clusters that were unique to session three could be attributed to unspecific effects of the mean intensity adjustment, analysis of session two was repeated with mean intensity adjustment but revealed no additional activation in the PCC, left insula and striatum (supplementary Table SIII). Overall, connectivity between the target region and the neurofeedback network instead decreased, suggesting that task-relevant variance was excluded by the mean intensity adjustment in this case.

Second, the exploratory analysis on instantaneous connectivity of the neurofeedback target region within groups as well as within sessions (supplementary Figure SII) revealed slightly higher overall connectivity of the target region during motor imagery / SMA neurofeedback (CHA group) compared to emotion regulation / aINS neurofeedback (CON group) during session one. Over time (sessions one to three), there was no visible trend difference between both groups. In the MIA corrected data (session two to three, CHA group) a modulatory influence of MIA was visible: In session two, MIA led to overall reduced connectivity to the neurofeedback network (see also supplementary Table SIII), additionally supporting that MIA reduced relevant variance in session two. In session three, MIA 
had the opposite effect, i.e. artificially low significance at $p=0.1$ disappeared after MIA and overall connectivity to the neurofeedback network increased strongly.

\begin{tabular}{|c|c|c|c|c|c|c|}
\hline Region & Center & of & mass & Extension & & \\
\hline Session 2 & $\mathbf{x}$ & $\mathbf{y}$ & $\mathbf{z}$ & Voxel & Session 2 & Session 3 \\
\hline TPJ R & 61.27 & -38.48 & 27.59 & 1308 & 5.13 & 3.96 \\
\hline MTG / Angular Gyrus L & -47.17 & -69.45 & 12.18 & 3323 & 5.35 & 3.27 \\
\hline TPJ L & -61.49 & -41.67 & 23.81 & 1159 & 5.36 & 3.46 \\
\hline
\end{tabular}

Session 3
\begin{tabular}{|l|c|c|c|c|c|c|}
\hline PCC & -0.06 & -47.16 & 40.95 & 1148 & 4.03 & 5.23 \\
\hline aINS / Striatum L & -25.56 & 13.97 & 1.74 & 2495 & 2.86 & 5.25 \\
\hline MTG R & 52.19 & -32.57 & 1.47 & 1989 & 3.60 & 5.30 \\
\hline
\end{tabular}

Shared between session
\begin{tabular}{|l|c|c|c|c|c|c|}
\hline aINS / vlPFC / dIPFC & 36.47 & 32.3 & 18.19 & 4036 & 5.40 \\
\hline OC / Cerebellum R & 25.04 & -56.56 & -12.89 & 134 & 4.95 & 5.48 \\
\hline Precuneus & -19.65 & -67.48 & 39.03 & 1097 & 5.51 & 5.10 \\
\hline ACC & 2.68 & 19.62 & 41.69 & 271 & 4.96 & 5.40 \\
\hline OC / Cerebellum L & -32.25 & -76.19 & -13.48 & 865 & 5.56 & 5.15 \\
\hline dLPFC L & -30.04 & 39.82 & 29.17 & 559 & 5.19 & 5.42 \\
\hline
\end{tabular}

Table 7: Instantaneous connectivity results for session two and three. The table contains location, extension and statistical properties of clusters with significant connectivity to the neurofeedback target region (aINS) during neurofeedback periods, cluster corrected at $p<$ 0.001 , for connectivity during Session two and three. Remarks: L, left; $R$, right, otherwise bilateral. 
A
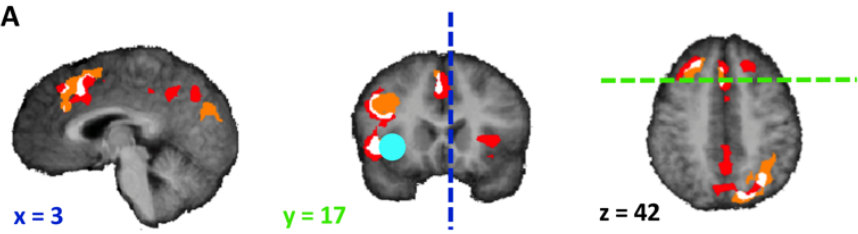

\section{Session 2}
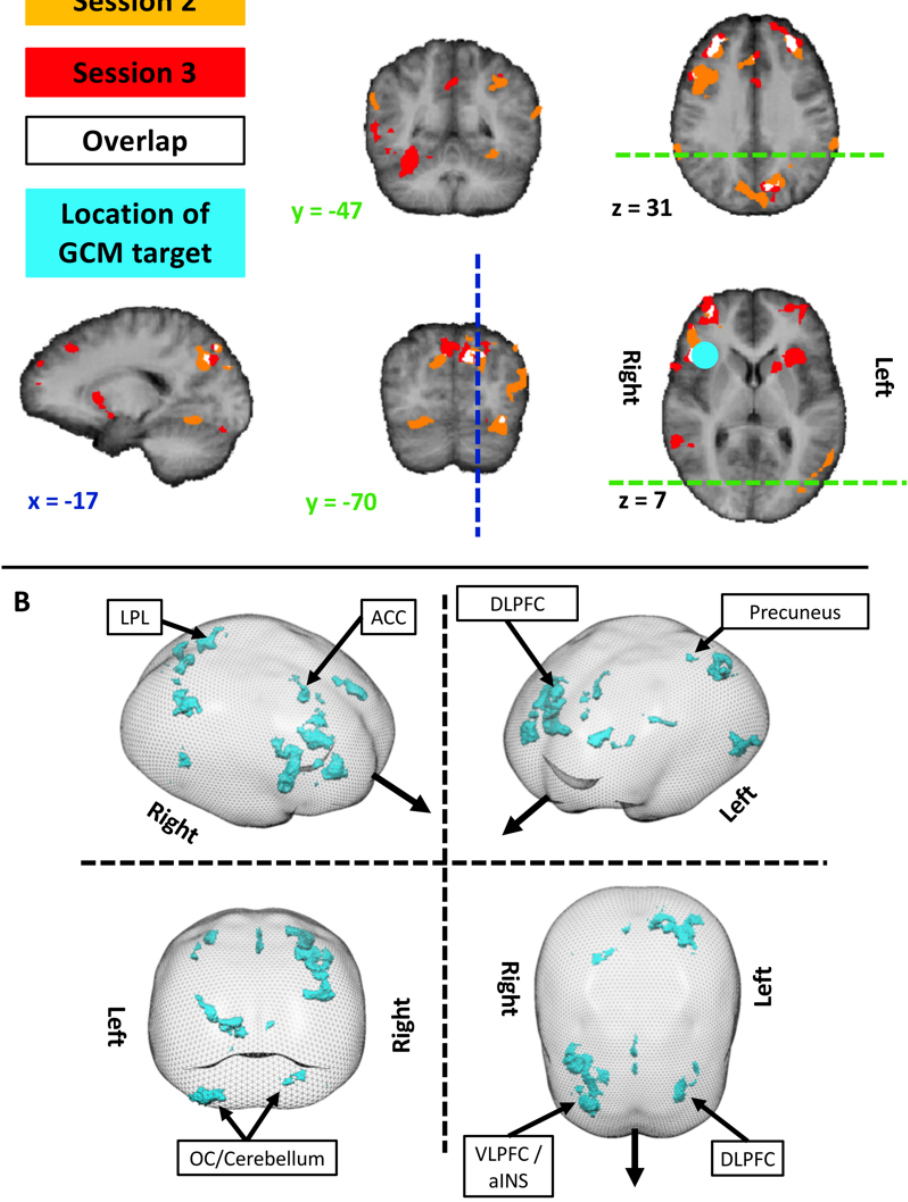

Figure 5: Instantaneous connectivity of the neurofeedback target regions during aINS neurofeedback. A. Significant connectivity of the neurofeedback target region (right alNS, turquoise) across both training schemes during emotion-regulation neurofeedback overlaid on the average group anatomy. During session two (orange) and three (red), the alNS showed connectivity to cortical hubs of the neurofeedback network (overlap between sessions: white). Sessions differed in which DMN hubs showed connectivity to the target region, and during session three the target region showed additional connectivity to the left 
aINS and anterior striatum. B. Three-dimensional reconstructions of connectivity clusters shared between session two and three. Across both sessions, the target region showed extensive connectivity to cortical regions implicated in cognitive control (dIPFC, vIPFC, aINS, $A C C$ and $L P L)$, visual feedback processing (ACC, OC) and a core hub of the DMN (precuneus).

\section{Discussion}

\subsection{Summary}

This exploratory project investigated the effects of neurofeedback training on whole-brain activation, connectivity of the neurofeedback target region and the stability of involved networks across different neurofeedback tasks and time.

By analysing functional activation during motor imagery SMA neurofeedback and emotion-regulation alNS neurofeedback, we could replicated recent metaanalytical findings that revealed a domain-general network of regions involved in neurofeedback guided self-regulation, including prefrontal / parietal regions associated with cognitive control and subcortical / occipital regions associated with feedback processing, while we additionally were able to dissociate task-specific regions selectively involved in emotion regulation and motor-imagery neurofeedback (Figure 1, Figure 2). By analysing instantaneous connectivity pattern within and across these two neurofeedback modalities, we could show that regions with higher activation during neurofeedback compared to rest also show increased connectivity in the early phase of neurofeedback training (Figure 3).

Over time, activation pattern compared to rest remained largely constant (Figure 4). Specifically the network of regions reliably activated across different 
neurofeedback tasks appeared to be stable over time, with slight modulations in subcortical areas by training style, i.e. a trend for striatum activation being more pronounced in the group training with a changing neurofeedback task. The main difference between session two and three was more pronounced deactivation of the default mode network during the last session that was shared between groups.

In accordance, instantaneous connectivity between the target region and the rest of the brain remained stable over the course of training, with differences between sessions two and three only being pronounced in connectivity to the $\mathrm{DMN}$, as well as increased connectivity to the striatum during the last session (Figure 5). Exploratory analysis of connectivity split between groups revealed that over time, extensive whole-brain connectivity of the aINS was observed for both groups at the end of training.

\subsection{Mental-task specific mechanisms contributing to neurofeedback trainings}

Interestingly, while both tasks shared a defined network of regions, motor specific activation could be observed during the SMA neurofeedback task: Here, activation in the posterior parietal lobe, the cerebellum and the brainstem increased, all regions associated with motor imagery (Jahn et al., 2008; Marchesotti et al., 2017) and with regard to the cerebellum and the posterior parietal lobe, commonly active during motor imagery during motor-imagery-guided control of brain-computer interfaces (Marchesotti et al., 2017).

The observed pronounced activation increases in fronto-polar regions during emotion regulation are in turn in line with a vast body of research suggesting the 
crucial involvement of anterior prefrontal areas as in emotion regulation (Engen, Kanske, \& Singer, 2016; Etkin, Büchel, \& Gross, 2015; Ochsner, Silvers, \& Buhle, 2012), and the continues involvement of lateral prefrontal areas during emotion regulation in both groups supports this notion. Additionally, in accordance with altered DMN and occipital activation during emotion regulation in session one, a recent large scale study on endogenous generation of affective states has reliably shown modulations in these areas and suggests that such activation could reflect a locus of attention directed towards internal processes (Engen et al., 2016). However, since the constant training group achieved on average significantly lower feedback magnitudes, especially during session one (see chapter 2 for results on feedback magnitude), both groups differ with regard to received visual stimulation which could have influenced occipital activation.

With regard to connectivity, selective differences were less pronounced. But the trend towards higher general connectivity of the SMA compared to the aINS during the first session (shown in the exploratory analysis), could reflect increased taskrelevant functional interactions between the SMA target area to regions involved in neurofeedback processing and self-regulation. Potentially, such high connectivity of the neurofeedback target region in the beginning of a training could be a marker for early self-regulation success, supported by the superior task performance during motor imagery.

Subsequently, pooling both tasks revealed strong connectivity of the neurofeedback network to the target areas, which overlapped with activation increases and were located near meta-analytic peak voxel (Emmert et al., 2016). Considering that activation increases in the neurofeedback network have been observed across several different neurofeedback tasks (Emmert et al. (2016), 
chapter 2 and 3), it could be expected, but is nevertheless remarkable, that pooling connectivity pattern from functionally very different regions as the aINS and the SMA are in such high accordance with activation increases observed across a range of neurofeedback tasks: First, this demonstrates the consistency of the neurofeedback network across different neurofeedback tasks with an additional neural marker, i.e. connectivity. Second, it shows how reliable neurofeedback works in guiding self-regulation by coupling nodes of the same network even to functionally very different neurofeedback target areas, and even when different mental task are used.

But despite shared activation and connectivity, participants could control the SMA neurofeedback better than the aINS neurofeedback (see chapter 2 ), so that one could expect the SMA group to have achieved a higher degree of synchronicity between activation in the neurofeedback target region and other brain regions involved in neurofeedback processing and self-regulation. While our (exploratory) between group analysis supports this notion by showing overall higher connectivity of the SMA than the aINS training during session one, it cannot be differentiated between connectivity differences resulting from the use of different mental tasks and differences caused by differences in task demand during the first session.

\subsection{Changes in the neurofeedback network over time}

Although activation remained largely stable across time, whole-brain analysis revealed modulated DMN activation during the last session accompanied by alterations in which hubs of the DMN connected to the neurofeedback target region. Given the crucial role of the DMN in processing of self-referential thoughts (Buckner, Andrews-Hanna, \& Schacter, 2008; Buckner \& Carroll, 2007) and the 
endogenous generation of emotional states (Engen et al., 2016), this could be related to changes in introspection and emotional self-regulation. Furthermore, particularly the decreased activation of the DMN could reflect more focused task engagement of participants, as the $\mathrm{DMN}$ is considered to infer with processing in the external attentional system (Fornito, Harrison, Zalesky, \& Simons, 2012).

On the other hand, prefrontal and parietal activation clusters of the neurofeedback network remained active across sessions and training styles, suggesting a crucial role of these regions across different stages of learning. Notably, the subcortical cluster of the neurofeedback network showed a trend for stronger striatal activation in the CHA group, which could reflect increased up-regulation of the striatum as part of the network involved in positive affect (Engen et al., 2016), in accordance with the higher mean neurofeedback magnitude of the $\mathrm{CHA}$ group (see chapter 2) or a more rewarding neurofeedback experience.

Additionally, instantaneous connectivity between the striatum and the target region was observed across both groups during the last session but not in session two. As discussed in chapter 2, task performance also significantly improved from session two to three (decreased distance between the neurofeedback signal and target level) so that participants received more positive feedback over time. Increased connectivity between the striatum and the target region could therefore reflect associative learning between rewarding experience and modulations in target region activation. Taking into account the consistency of target region connectivity to prefrontal and parietal control areas, this suggests that regions in the network described by Emmert et al. (2016) synchronize their activation with activation of the target region during neurofeedback. However, the functional 
relevance of such synchronicity during neurofeedback will have to be investigated in future research.

\subsection{Limitations and future directions}

By gaining insight into the instantaneous connectivity of the neurofeedback target region, i.e. functional connectivity that excludes major directional influences, we revealed regions which synchronize their activation during neurofeedback with the neurofeedback target region as visible at the sampling rate of $1 \mathrm{sec}$. However, it is worth mentioning that while instantaneous connectivity can provide a reliable estimate of joint activation between regions below the sampling frequency, directional influences above the sampling frequency cannot be excluded and therefore could contribute to our findings (for a discussion on this matter see Goebel et al. (2003)). Future research additionally applying electrophysiological measurements should further expand on these findings, to determine how far the observed fMRI connectivity profile still includes non-instantaneous components which cannot be excluded due to the temporal resolution of fMRI.

Overall, it remains to be noted that the gathered results stem from a small sample and our exploratory observations must therefore be interpreted with caution. This unfortunately affected the possibility to detect statistically significant effects that required considerable power as RFX comparisons between neurofeedback modalities and timepoints. A specific conclusion to be drawn from our observations is, therefore, that the previously reported neurofeedback network (Emmert et al., 2016) can be detected in small groups of participants at different training stages. The spatial consistency of recruited brain areas thereby makes the neurofeedback network itself a potential target for neuromodulation techniques. Targeting the neurofeedback network with for example neurostimulation could help to facilitate 
the effects of neurofeedback in the future. To further investigate interactions between task difficulty, learning progression, connectivity and activation, future studies should analyse effects of task difficulty over longer periods of time and with larger sample sizes.

\subsection{Conclusion}

Overall, the acquired results allow several conclusions: Firstly, activation in a core network of regions recruited during neurofeedback is not only stable across different neurofeedback tasks, but also over time. Secondly, activation in this core network is complemented by additional activation in task-specific regions. Thirdly, activation in this network is not a mere reflection of independent neural processes, but involved regions synchronize their activation with activation in the neurofeedback target region. Fourthly, while over time the same set of key regions is recruited, the implicated network is fine-tuned in response to the training.

On the one hand, the activation pattern emerging during neurofeedback therefor constitutes a neural mechanism, which shows highly stable behaviour. The recruited network thereby flexibly adapts to different characteristics of the training situation and undergoes subtle changes in connectivity and activation in response to the training. Neurofeedback therefor involves a predictable neural mechanisms that can flexibly be applied to a variety intervention settings. 


\section{References}

Borm, G. F., Hoogendoorn, E. H., den Heijer, M., \& Zielhuis, G. A. (2005). Sequential balancing: a simple method for treatment allocation in clinical trials. Contemporary clinical trials, 26(6), 637-645.

Büchel, C., Coull, J., \& Friston, K. (1999). The predictive value of changes in effective connectivity for human learning. Science, 283(5407), 1538-1541.

Buckner, R. L., Andrews-Hanna, J. R., \& Schacter, D. L. (2008). The brain's default network. Annals of the New York Academy of Sciences, 1124(1), 1-38.

Buckner, R. L., \& Carroll, D. C. (2007). Self-projection and the brain. Trends in cognitive sciences, 11(2), 49-57.

Daw, N. D., Niv, Y., \& Dayan, P. (2005). Uncertainty-based competition between prefrontal and dorsolateral striatal systems for behavioral control. Nature neuroscience, 8(12), 1704.

Emmert, K., Kopel, R., Sulzer, J., Brühl, A. B., Berman, B. D., Linden, D. E., . . Frank, S. (2016). Meta-analysis of real-time fMRI neurofeedback studies using individual participant data: How is brain regulation mediated? Neurolmage, 124, 806-812.

Engen, H. G., Kanske, P., \& Singer, T. (2016). The neural component-process architecture of endogenously generated emotion. Social cognitive and affective neuroscience, 12(2), 197-211.

Etkin, A., Büchel, C., \& Gross, J. J. (2015). The neural bases of emotion regulation. Nature Reviews Neuroscience, 16(11), 693.

Fornito, A., Harrison, B. J., Zalesky, A., \& Simons, J. S. (2012). Competitive and cooperative dynamics of large-scale brain functional networks supporting recollection. Proceedings of the National Academy of Sciences, 109(31), 12788-12793.

Freund, P. A., Kuhn, J.-T., \& Holling, H. (2011). Measuring current achievement 
motivation with the QCM: Short form development and investigation of measurement invariance. Personality and Individual Differences, 51(5), 629-634.

Friston, K., Fletcher, P., Josephs, O., Holmes, A., Rugg, M., \& Turner, R. (1998). Eventrelated fMRI: characterizing differential responses. Neurolmage, 7(1), 3040.

Friston, K. J. (2011). Functional and effective connectivity: a review. Brain connectivity, 1(1), 13-36.

Friston, K. J., Harrison, L., \& Penny, W. (2003). Dynamic causal modelling. Neuroimage, 19(4), 1273-1302.

Gerchen, M. F., \& Kirsch, P. (2017). Combining task-related activation and connectivity analysis of $\mathrm{fMRI}$ data reveals complex modulation of brain networks. Human Brain Mapping, 38(11), 5726-5739.

Goebel, R., Roebroeck, A., Kim, D.-S., \& Formisano, E. (2003). Investigating directed cortical interactions in time-resolved fMRI data using vector autoregressive modeling and Granger causality mapping. Magnetic resonance imaging, 21(10), 1251-1261.

Haller, S., Kopel, R., Jhooti, P., Haas, T., Scharnowski, F., Lovblad, K.-O., . . Van De Ville, D. (2013). Dynamic reconfiguration of human brain functional networks through neurofeedback. Neurolmage, 81, 243-252.

Jahn, K., Deutschländer, A., Stephan, T., Kalla, R., Wiesmann, M., Strupp, M., \& Brandt, T. (2008). Imaging human supraspinal locomotor centers in brainstem and cerebellum. Neurolmage, 39(2), 786-792.

Johnston, S., Linden, D., Healy, D., Goebel, R., Habes, I., \& Boehm, S. (2011). Upregulation of emotion areas through neurofeedback with a focus on positive mood. Cognitive, Affective, \& Behavioral Neuroscience, 11(1), 4451. 
Kim, D.-Y., Yoo, S.-S., Tegethoff, M., Meinlschmidt, G., \& Lee, J.-H. (2015). The inclusion of functional connectivity information into fMRI-based neurofeedback improves its efficacy in the reduction of cigarette cravings. Journal of cognitive neuroscience, 27(8), 1552-1572.

Lang, P. J., Bradley, M. M., \& Cuthbert, B. N. (1997). International affective picture system (IAPS): Technical manual and affective ratings. NIMH Center for the Study of Emotion and Attention, 39-58.

Lee, S., Ruiz, S., Caria, A., Veit, R., Birbaumer, N., \& Sitaram, R. (2011). Detection of cerebral reorganization induced by real-time fMRI feedback training of insula activation: a multivariate investigation. Neurorehabilitation and neural repair, 25(3), 259-267.

Liew, S.-L., Rana, M., Cornelsen, S., Fortunato de Barros Filho, M., Birbaumer, N., Sitaram, R., . . Soekadar, S. R. (2016). Improving motor corticothalamic communication after stroke using real-time fMRI connectivity-based neurofeedback. Neurorehabilitation and neural repair, 30(7), 671-675.

Marchesotti, S., Martuzzi, R., Schurger, A., Blefari, M. L., del Millán, J. R., Bleuler, H., \& Blanke, O. (2017). Cortical and subcortical mechanisms of brain-machine interfaces. Human Brain Mapping, 38(6), 2971-2989.

Megumi, F., Yamashita, A., Kawato, M., \& Imamizu, H. (2015). Functional MRI neurofeedback training on connectivity between two regions induces longlasting changes in intrinsic functional network. Frontiers in human neuroscience, 9.

Murphy, K., \& Fox, M. D. (2017). Towards a consensus regarding global signal regression for resting state functional connectivity MRI. Neurolmage, 154, 169-173.

Ochsner, K. N., Silvers, J. A., \& Buhle, J. T. (2012). Functional imaging studies of emotion regulation: a synthetic review and evolving model of the cognitive 
control of emotion. Annals of the New York Academy of Sciences, 1251(1), E1-E24.

Roebroeck, A., Formisano, E., \& Goebel, R. (2005). Mapping directed influence over the brain using Granger causality and fMRI. Neuroimage, 25(1), 230-242.

Rota, G., Handjaras, G., Sitaram, R., Birbaumer, N., \& Dogil, G. (2011). Reorganization of functional and effective connectivity during real-time fMRI-BCI modulation of prosody processing. Brain and language, 117(3), 123-132.

Sitaram, R., Ros, T., Stoeckel, L., Haller, S., Scharnowski, F., Lewis-Peacock, J., . . . Oblak, E. (2017). Closed-loop brain training: the science of neurofeedback. Nature reviews neuroscience, 18(2), 86.

Sorger, B., Kamp, T., Weiskopf, N., Peters, J. C., \& Goebel, R. (2016). When the brain takes 'BOLD'steps: real-time fMRI neurofeedback can further enhance the ability to gradually self-regulate regional brain activation. Neuroscience.

Sporns, O. (2011). Brain Networks: Structure and Dynamics Networks of the Brain: MIT press.

Talairach, J., \& Tournoux, P. (1988). Co-planar Stereotaxic Atlas of the Human Brain: 3-dimensional Proportional System : an Approach to Cerebral Imaging: Thieme.

Watson, D., Clark, L. A., \& Tellegen, A. (1988). Development and validation of brief measures of positive and negative affect: the PANAS scales. Journal of personality and social psychology, 54(6), 1063.

Weissenbacher, A., Kasess, C., Gerstl, F., Lanzenberger, R., Moser, E., \& Windischberger, C. (2009). Correlations and anticorrelations in resting-state functional connectivity MRI: a quantitative comparison of preprocessing strategies. Neurolmage, 47(4), 1408-1416.

Yamashita, A., Hayasaka, S., Kawato, M., \& Imamizu, H. (2017). Connectivity 
Neurofeedback Training Can Differentially Change Functional Connectivity and Cognitive Performance. Cerebral Cortex, 27(10), 4960-4970. 


\section{Supplementary Material}

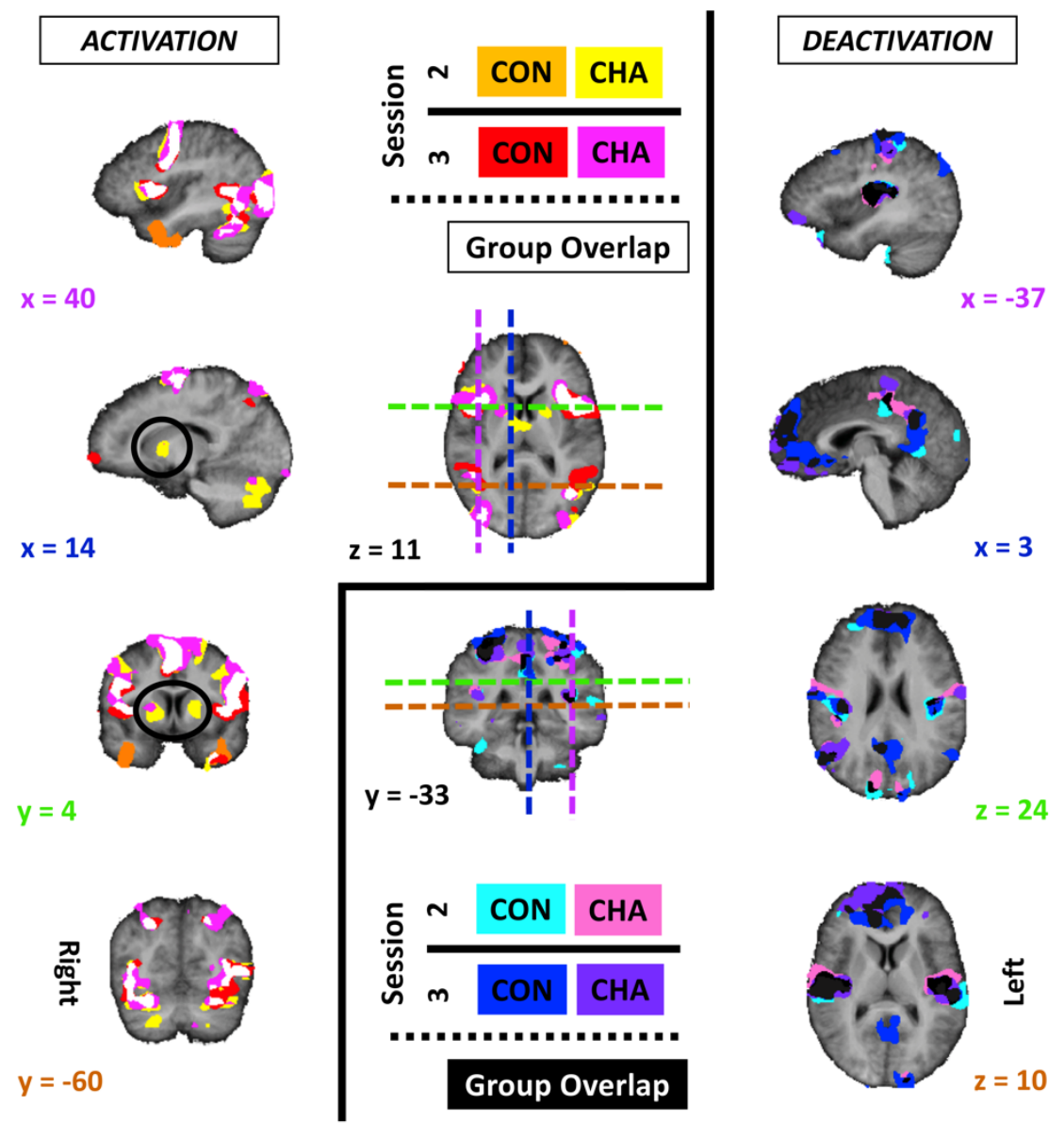

Figure SI. Whole-brain activation (left) and deactivation (right) for neurofeedback compared to rest for session two and three split between groups (FFX results). Note that only the CHA group shows significantly increased striatum activation, results are Bonferroni corrected at $p=0.001$. 

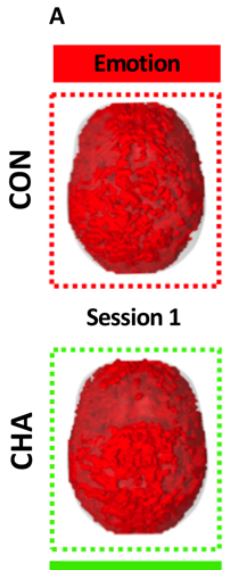

Motor Imagery

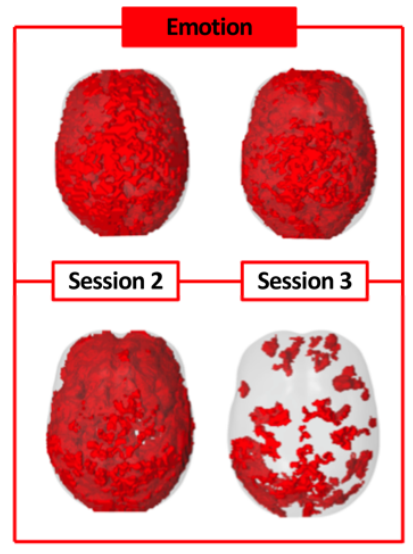

$\mathrm{p}<0.1$
B

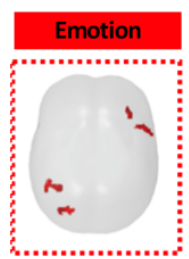

Session 1

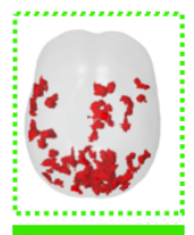

Motor Imagery

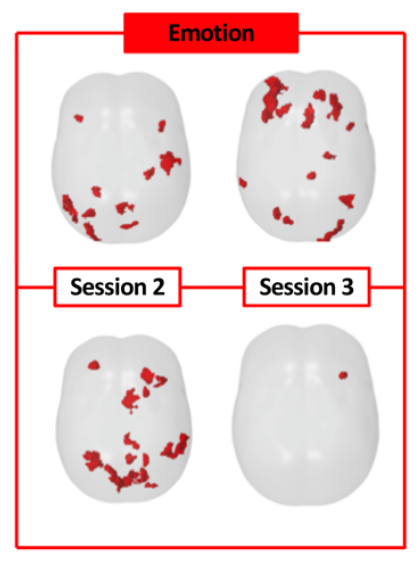

$$
p<0.005
$$

D

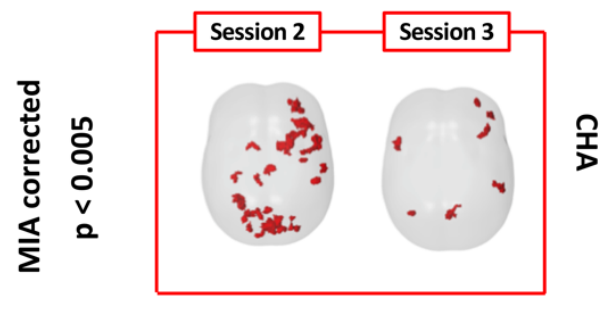

Figure SII. Exploratory analysis of instantaneous connectivity of neurofeedback target regions. $A$ and $B$ show the effect of thresholding $(p<0.01$ and $p<0.005)$ on connectivity before adjustment for mean intensity (MIA) for alNS neurofeedback (red outline) and SMA neurofeedback (green outline). During session three the CHA group showed low significance even during overly liberal thresholding compared to all other group time-points. $C$ and $D$ show group results after mean intensity adjustment (MIA) for P08 (participant of the CHA group) who showed a pronounced influence of global intensity fluctuations on instantaneous connectivity. The dataset of the corresponding participant in session two was additionally reprocessed with MIA for comparability of emotion-regulation training effects. After MIA, analysis of session two showed a slight loss in statistical sensitivity; for session three sensitivity significantly improved (see also Table SIII). A cluster threshold of 200 structural voxel was applied to all group time-points. 


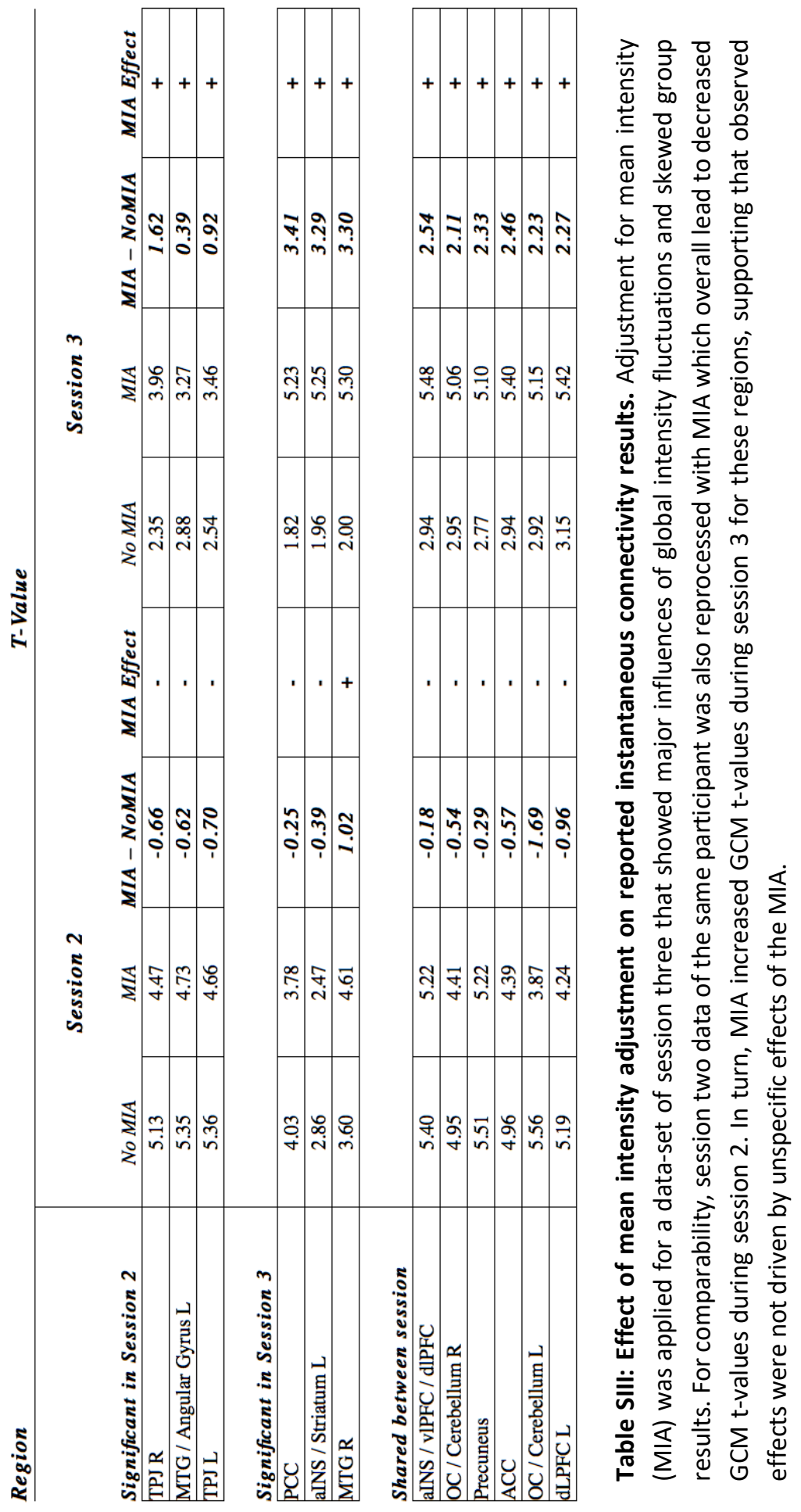






\section{Chapter 4 - Success and failure of controlling the rtfMRI neurofeedback signal are reflected in the striatum}

Based on:

Skottnik, L., Sorger, B., Kamp, T., Linden, D., \& Goebel, R. (2019). Success and failure of controlling the real-time functional magnetic resonance imaging neurofeedback signal are reflected in the striatum. Brain and Behavior, 9(3), e01240. 



\section{Abstract}

Over the last decades, neurofeedback has been applied in variety of research contexts and therapeutic interventions. Despite this extensive use, its neural mechanisms are still under debate. Several scientific advances have suggested that different networks become jointly active during neurofeedback, including regions generally involved in self-regulation, regions related to the specific mental task driving the neurofeedback and regions generally involved in feedback learning (Sitaram et al., 2017). To investigate the neural mechanisms specific to neurofeedback but independent from general effects of self-regulation, we compared brain activation as measured with functional magnetic resonance imaging (fMRI) across different mental tasks involving gradual self-regulation with and without providing neurofeedback. Ten participants freely chose one selfregulation task and underwent two training sessions during fMRI scanning, one with and one without receiving neurofeedback. During neurofeedback sessions, feedback signals were provided in real-time based on activity in task-related, individually defined target regions. In both sessions, participants aimed at reaching and holding low, medium or high brain-activation levels in the target region. During gradual self-regulation with neurofeedback, a network of cortical control regions as well as regions implicated in reward and feedback processing were activated. Selfregulation with feedback was accompanied by stronger activation within the striatum across different mental tasks. Additional time-resolved single-trial analysis revealed that neurofeedback performance was positively correlated with a delayed brain response in the striatum that reflected the accuracy of self-regulation. Overall, these findings support that neurofeedback contributes to self-regulation through task-general regions involved in feedback- and reward processing. 


\section{Introduction}

Despite its extensive use over several decades and broad evidence for neurofeedback induced changes that extend beyond the neurofeedback training environment (including on memory (Young et al., 2017), affect (Scheinost et al., 2013; Zilverstand, Sorger, Sarkheil, \& Goebel, 2015), attention (Zilverstand et al., 2017), perception (Amano, Shibata, Kawato, Sasaki, \& Watanabe, 2016) and motor performance (Subramanian et al., 2011)) the neural mechanisms underlying neurofeedback are subject of an ongoing debate (for an overview see Sitaram et al. (2017)). In a recent meta-analysis, whole-brain activation during real-time functional magnetic resonance imaging (rtfMRI) neurofeedback was compared across different neurofeedback studies (Emmert et al., 2016). Activation during neurofeedback training was observed in areas implicated in self-regulation and cognitive control, as well as in areas recruited during visual feedback learning, even if these areas were not actually the target of the self-regulation training. The activated network encompassed the dorsolateral (DLPFC) and ventrolateral prefrontal cortex (VLPFC), the temporo-parietal cortex and the thalamus, anterior insula (aINS), the posterior section of the anterior cingulate cortex (pACC), visual areas and the basal ganglia, with several local maxima distributed over the striatum. Activity in these regions most likely reflects several different processes, including the preparation and execution of mental strategies supporting selfregulation of brain activity, reward processing, self-evaluation of performance based on feedback information and the updating of strategies, but an extensive body of research is still needed to disentangle these processes. To discriminate the neural basis of neurofeedback from networks also recruited during other forms of self-regulation training, it remains to be understood which regions shared between different neurofeedback tasks are specific to neurofeedback and which are reflective of self-regulation per se. 
Marchesotti et al. (2017) detected a selective activation increase in the striatum during motor imagery with neurofeedback when comparing meta-analytic activation maps of motor imagery with and without providing neurofeedback and Johnston, Boehm, Healy, Goebel, and Linden (2010) had reported increased activation in the ventral striatum with progression in neurofeedback training for up-regulating negative affect by providing neurofeedback from individual areas that showed increased activation in response to negative affective image. In congruence with these reported activation increases in the striatum during neurofeedback, several theoretical frameworks note that $\mathrm{BCl}$ control/neurofeedback rewards subjects for a certain mental operation or neural state, notably by underling the crucial involvement of operant/instrumental conditioning in neurofeedback (Fetz, 2007), by interpreting BCl control training as skill learning that is heavily dependent on plasticity in the basal ganglia (Birbaumer, Ruiz, \& Sitaram, 2013) or by underlining the importance of feedback loops for biofeedback learning in general (Lacroix \& Gowen, 1981) While early EEGneurofeedback studies lacked direct evidence for involvement of the striatum in neurofeedback due to the limitations of EEG in coverage of subcortical areas (Grech et al., 2008), contemporary approaches on EEG and fMRI neurofeedback agree with regard to the central role of striatal reward learning (Birbaumer et al., 2013; Davelaar, 2018).

In the present study, we extended the aforementioned line of research by comparing self-regulation with and without neurofeedback with a special focus on the striatum, a key region involved in feedback and reward processing (Balleine, Delgado, \& Hikosaka, 2007; Bartra, McGuire, \& Kable, 2013; Kohrs, Angenstein, Scheich, \& Brechmann, 2012), the central hub of dopamine based reinforcement 
learning (Robbins \& Everitt, 1996) where feedback information is processed and further utilized to guide actions (O'Doherty et al., 2004; Samejima, Ueda, Doya, \& Kimura, 2005) and constituting the main hub for long term motivation of behaviour based on reward learning (Tricomi, Balleine, \& O'Doherty, 2009), making the understanding of how neurofeedback affects the striatum a crucial element of understanding the facilitating effects of neurofeedback in general.

As neurofeedback is most commonly used to guide a participant's self-regulation by reinforcing activation states via operant conditioning with positive feedback (Birbaumer et al., 2013; Fetz, 2007) , we therefor predicted that striatum activation would constitute a crucial marker for differentiating between self-regulation with neurofeedback and self-regulation without neurofeedback, as it reflects external reward information that is utilized to guide ongoing behaviour (Balleine et al., 2007), which is lacking in self-regulation without neurofeedback.

While the striatum is a functionally heterogeneous structure (parcellation studies suggest that ventral/anterior portions are more strongly involved in evaluating incoming reward, whereas medial to dorsal sections rather bias actions based on previously processed rewards (Balleine et al., 2007; Jung et al., 2014; O'Doherty et al., 2004), different functional processes in the striatum transition smoothly into each other (Haber, Fudge, \& McFarland, 2000) and timing of incoming rewards constitutes a crucial influence across different processing stages in the striatum (Cardinal, 2006; Gustavo, Soares, \& Paton, 2015; McClure, Berns, \& Montague, 2003; Pagnoni, Zink, Montague, \& Berns, 2002) So while previous studies have mainly concentrated on localizing the regions involved in neurofeedback, we additionally applied a time-resolved analysis on the blood oxygen level dependent 
(BOLD) signal of the striatum, to determine the temporal properties of feedback processing.

Up to this date, most neurofeedback paradigms focused on decreasing or increasing activation within a certain brain region (Caria et al., 2007; Hamilton, Glover, Hsu, Johnson, \& Gotlib, 2011), functional connectivity between brain regions (Megumi, Yamashita, Kawato, \& Imamizu, 2015), directional connectivity between brain regions (Haller et al., 2013), or frequency-bands (Gevensleben et al., 2009; Mottaz et al., 2015). These paradigms reinforced subjects to modulate the neurofeedback signal into one direction, i.e., to either up- or down-regulate the neurofeedback signal maximally. We recently demonstrated feasibility of a novel type of neurofeedback paradigm in which participants focused on achieving and maintaining a specific target level of activation (Sorger, Kamp, Weiskopf, Peters, \& Goebel, 2016): Participants aimed at reaching/maintaining a rtfMRI-neurofeedback signal (visualized by means of a thermometer display) corresponding to the brainactivation level within individually defined brain regions at either $30 \%, 60 \%$ or $90 \%$ of their individual maximal activation capacity. We found that participants showed a significantly increased ability to gradually self-regulate activation in the neurofeedback target regions, when receiving visually presented neurofeedback information compared to gradual self-regulation without providing neurofeedback. In contrast to classical paradigms that train to maximize (de)activation or connectivity, participants trained according to the novel parametric activation paradigm received detailed neurofeedback information on the current brainactivation level with every data point (here every $2 \mathrm{~s}$ ) visualized as deviation of the actual condition (actually achieved brain-activation level) from the different nominal conditions (instructed target brain-activation levels). Moreover, they could deviate from the task goal by both reaching too high or too low activation levels 
(not given in the conventional, maximization paradigms). These features considerably increase the general task difficulty, and we would expect that successful task performance is being experienced as strongly rewarding (see DePasque Swanson and Tricomi (2014)). Another advantage of gradual feedback for studies into the mechanisms of self-regulation is that the visual information provided during neurofeedback is more important for successful task performance than in maximization paradigms, as participants not only need to learn how activation could be increased or decreased best, but also how the actual magnitude of activation can be held at a particular target level. Gradual feedback protocols are thus particularly suited for studies that look into the learning mechanisms underlying successful neurofeedback training.

In the present study, we defined and applied a novel marker of self-regulation success to a dataset from the aforementioned self-regulation study by Sorger et al. (2016). This marker of self-regulation success represents the neurofeedback reward value as indicated by the visual information on the feedback display. In the study of Sorger et al. (2016), each participant chose one individual mental task for self-regulation and all participants trained to self-regulate their engagement with the chosen mental content gradually (chosen mental tasks included inner speech, motor imagery, mental calculation, visual imagery and auditory imagery). The interindividual heterogeneity of self-regulation strategies allows investigating the shared neural basis of neurofeedback. Participants underwent two self-regulation sessions during $\mathrm{fMRI}$, one with and one without receiving feedback information from individually defined neurofeedback target regions. This allows us to control for effects of self-regulation that are unrelated to neurofeedback as for example observed during meditation (Kjaer et al., 2002; Tang, Hölzel, \& Posner, 2015), and reveal regions more related to the actual processing of neurofeedback and the 
implicated reward information. As the neurofeedback signal was provided continuously, the constant influx of feedback information created a demanding situation for the processing of reward information: Neurofeedback was constantly updated, while being delayed over several seconds in relation to the mental action actually causing a change in the neurofeedback signal. As activation in the striatum is known to be strongly influenced by the temporal properties of reward information (Cardinal, 2006; Gustavo et al., 2015; McClure et al., 2003; Pagnoni et al., 2002), analysis has to take the temporal sensitivity of reward processing into account. Analysis of the available data therefore focused on the dynamic and delayed nature of the reward information provided by rtfMRI neurofeedback. This was achieved by extracting one value of neurofeedback performance for every data point acquired during gradual self-regulation periods (every $2 \mathrm{~s}$ ) and by relating this information to striatum activation in different time windows.

Taking into consideration these ideas and in order to further study the neuronal mechanisms of rtfMRI neurofeedback, more particularly the role of the striatum, the present study focused on the following research objectives:

i. Demonstrate joint activation of cortical control areas and areas related to feedback learning within a single sample during neurofeedback-guided selfregulation compared to rest, thereby investigating the replicability of recent metaanalytical findings (combining data of several neurofeedback studies (Emmert et al., 2016)) and their reliability in smaller samples.

ii. Separate activation related to feedback processing from activation related to self-regulation during neurofeedback in the striatum and determine whether increased striatum activation during neurofeedback reflects a specific response to the information contained in the provided neurofeedback information. 
iii. Disentangle which activation increases during neurofeedback indicate feedback processing and which are reflective of higher-order cognitive control processes involved in self-regulation.

\section{Methods and Design}

\subsection{Participants}

All analysis was performed on the dataset acquired by Sorger et al. (2016): Ten healthy participants (mean age: 27.0 years, SD: 3.8 years, five female, one lefthanded), all students or staff members of the Faculty of Psychology and Neuroscience at Maastricht University with normal or corrected-to-normal vision participated in the study (see Sorger et al. (2016) for more detailed participant characteristics). None of the participants had participated in a neurofeedback experiment before. Before each MRI scanning session, participants gave written informed consent. The experimental procedure was approved by the local Ethics Committee of the Faculty of Psychology and Neuroscience at Maastricht University.

\subsection{Experimental procedure}

Preceding the first MRI measurement, each participant freely chose one individual mental task for self-regulation: Experimenters suggested various mental tasks (inner speech, motor imagery, mental calculation, visual imagery and auditory imagery) that had been proven to evoke robust brain activation in circumscribed brain regions in previous $\mathrm{fMRI}$ studies as possible activation strategies. Additionally, 
the experimenters recommended several modulation strategies that could be applied by participants to alter the brain-activation level. Basically, these strategies allowed for changing certain aspects of mental-task performance parametrically (e.g., the speed, intensity or complexity). Participants selected their activation strategies and initial modulation strategies based on personal preference or feeling of best mastery. Chosen self-regulation tasks included inner speech, motor imagery, auditory imagery, and visual imagery. Importantly, no participant used voluntary emotion regulation as mental strategy, thereby fore coming that alterations in striatum activation were dominated by voluntarily generated affective states.

Participants received no feedback in one fMRI session, whereas in the other session they were provided with real-time information on the current BOLD-signal level in a pre-defined mental task-related brain region. During neurofeedback sessions, participants were asked to modulate their BOLD signal to three different target levels using the chosen mental task. The no-feedback and feedback fMRI sessions took place on separate days for all participants. The order of the type-of-training conditions (no feedback-feedback or feedback-no feedback) was balanced across participants. Both scanning sessions consisted of four training (modulation) runs in which participants were visually instructed to modulate their BOLD-signal magnitude to the three different target levels. Each target-level condition appeared three times per run in randomized order resulting in a total of twelve trials per target-level and type-of-training condition. Each of the nine modulation blocks and each of the ten resting blocks that alternated with the modulation blocks lasted 26 $\mathrm{s}$ resulting in a run length of $8 \mathrm{~min}$ and $14 \mathrm{~s}$. A feedback scanning session started with a functional-localizer run in order to select a mental task-specific neurofeedback target region and to determine the individual maximum percent 
signal change (maxPSC). In the functional-localizer run, two target-levels (50\% and $100 \%)$ were implemented (five trials per target-level condition). The two targetlevel conditions appeared in alternating order. Again, the duration of the ten modulation trials and the eleven resting periods were $26 \mathrm{~s}$ adding up to a total run duration of 9 min and $6 s$.

\subsection{Visual stimulation and instructions}

Participants were instructed to keep their selected activation strategy constant across all functional runs (functional-localizer, no-feedback and feedback runs). Thus, they should not change their general activation strategy across time (and sessions). In order to modulate their BOLD signal to the different target levels, participants were asked to apply the modulation strategies. Importantly, in the feedback condition participants were instructed to consider the provided neurofeedback information and to explore which of the modulation strategies were most effective. Moreover, participants were explicitly allowed to adapt the suggested modulation strategies or even generate and test novel ('own') modulation strategies. During functional-localizer and no-feedback runs, participants were asked to try to evoke different brain-activation levels based on their current hypothesis on how the BOLD-signal magnitude can be altered systematically.

During self-regulation (with and without feedback), a thermometer-like display on black background was used consisting of ten white rectangles stacked on top of each other (see Figure 1). Participants were instructed to adjust their BOLD-signal magnitude to a particular target level by displaying the outline of a certain rectangle in red for the duration of the modulation trial. Thus, the vertical position of the 
colored rectangle represented the desired brain-activation target level. In the functional-localizer run, rectangle 5 (counted from bottom) corresponded to the $50-\%$ condition and rectangle 10 represented the $100-\%$ condition of the individual maxPSC. In the modulation runs, rectangles 3, 6, and 9, corresponded to the low, medium and high target-level conditions, respectively.

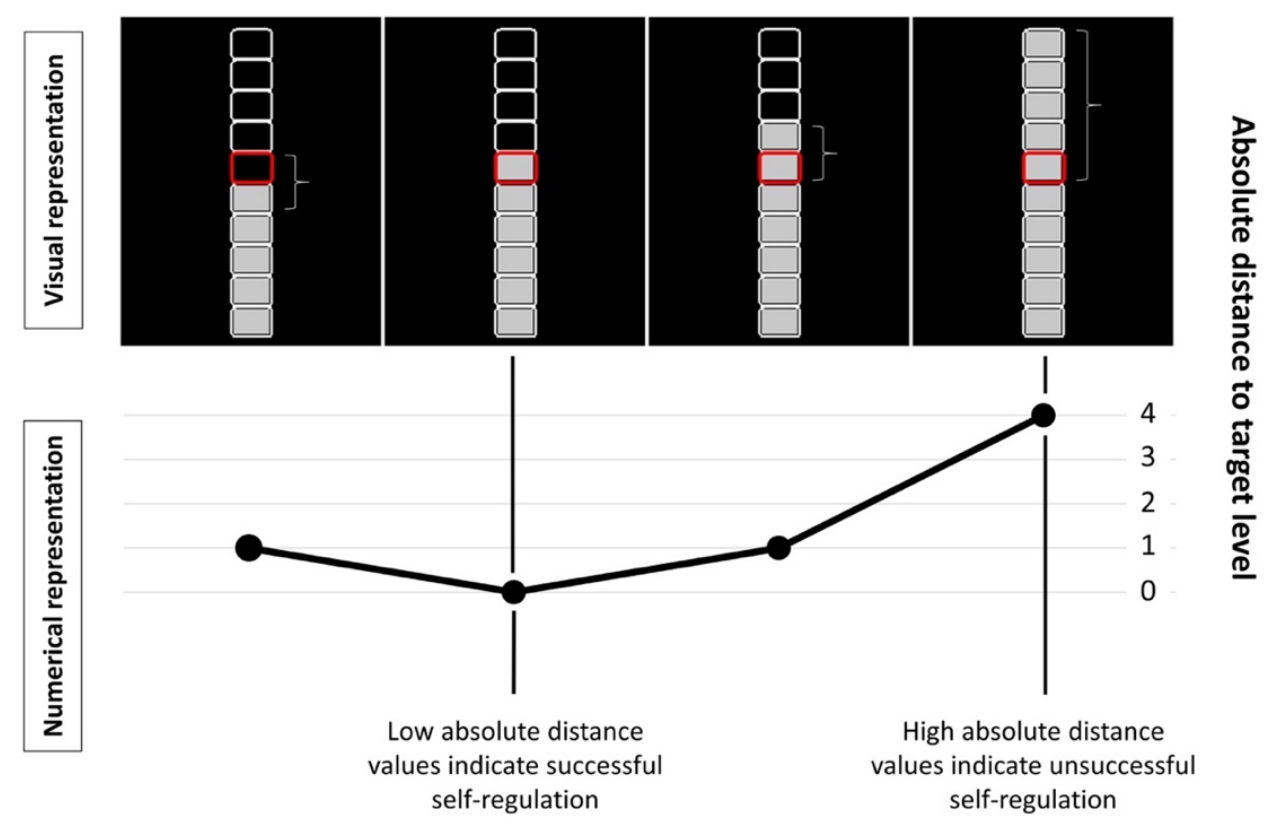

Figure 1

Absolute distance of achieved activation level to instructed target activation level.

Participants evaluated the appropriateness of their mental operation (and therewith their self-regulation success) based on the visually provided neurofeedback information. They could assess their self-regulation success by obtaining the absolute distance between the magnitude of the actually achieved activation level (provided neurofeedback information) and the instructed target activation level (indicated by the red rectangular). A smaller and larger distance to the target activation level represented a superior and inferior selfregulation performance, respectively. 
During resting periods, no rectangle was colored red. In the modulation runs of the feedback session, participants were additionally provided with continuously updated information about their current BOLD-signal level within the neurofeedback target region. This was realized by filling in (with gray color) the thermometer's rectangles according to the actual current BOLD-signal level within the neurofeedback target region. Participants were instructed to reach and hold the desired brain-activation target level, thereby reducing the absolute distance between the BOLD-signal level and the target rectangle (see Figure 1).

\subsection{Data acquisition}

(f)MRI data were acquired using a 1.5-T whole-body (Magnetom Sonata; Siemens AG, Erlangen, Germany) or a 3-T head scanner (Siemens Allegra, Siemens AG). Participants' heads were fixated with foam padding to minimize spontaneous or task-related motion. The proportion of participants undergoing 1.5 and 3T scanning was balanced (5/5) and each participant underwent the same field strength for both training sessions.

\section{Structural Data acquisition}

All participants received a high-resolution T1-weighted anatomical scan using a three-dimensional (3D) magnetization prepared rapid-acquisition gradient-echo (MPRAGE) sequence (1.5-T scanning: 192 slices, slice thickness $=1 \mathrm{~mm}$, no gap, repetition time $[T R]=2000 \mathrm{~ms}$, echo time $[T E]=3.93 \mathrm{~ms}$, flip angle $[F A]=15$, field of view $[\mathrm{FOV}]=250 \times 250 \mathrm{~mm} 2$, matrix size $=256 \times 256$, total scan time $=8 \mathrm{~min}$ and $34 \mathrm{~s}$; 3-T scanning: 192 slices, slice thickness $=1 \mathrm{~mm}$, no gap, $\mathrm{TR}=2250 \mathrm{~ms}, \mathrm{TE}=2.6$ $\mathrm{ms}, \mathrm{FA}=9, \mathrm{FOV}=256 \times 256 \mathrm{~mm} 2$, matrix size $=256 \times 256$, total scan time $=8 \mathrm{~min}$ and $26 \mathrm{~s})$. 


\section{Functional data acquisition}

Repeated single-shot echo-planar imaging (EPI) was performed. Except for the number of acquisitions (functional-localizer run: 273 volumes; modulation runs: 247 volumes), identical scanning parameters were used for all functional measurements $\left(T R=2000 \mathrm{~ms}, \mathrm{TE}=40 \mathrm{~ms}, \mathrm{FA}=90, \mathrm{FOV}=224 \times 224 \mathrm{~mm}^{2}\right.$, matrix size $=64 \times 64$, number of slices $=25$, slice thickness $=3 \mathrm{~mm}, 1 \mathrm{~mm}$ gap, slice order $=$ ascending/interleaved).

\section{Analysis}

\subsection{Neurofeedback preparation and generation}

\subsubsection{Selection and definition of neurofeedback target regions}

After completion of the functional-localizer run, the first two volumes were discarded from further analysis to account for T1-saturation effects. Functional data were then pre-processed (motion correction, linear-trend removal, temporal high-pass filtering [three cycles/time course]). Eventually, a multiple-regression general linear model (GLM) was calculated voxel-wise applying predictors corresponding to the two target-level conditions (predictor time courses being derived from a boxcar function convolved with a standard hemodynamic response function [single-gamma function (Boynton, Engel, Glover, \& Heeger, 1996)]. Candidate neurofeedback target regions were identified by contrasting the mean brain activation during both target-level conditions to the mean activation during the interleaved resting periods. From the obtained F-maps $(p<0.05$, Bonferronicorrected), a region of interest (ROI) was defined for each participant (for details of neurofeedback target regions see Sorger et al. (2016)). 


\subsubsection{Calculation of the feedback signal}

For an extensive description of how the neurofeedback signal was created the reader is referred to Sorger et al. (2016). In short, functional images were reconstructed and written to the scanner console's hard disk in real-time during neurofeedback sessions. The real-time data analysis software (Turbo-BrainVoyager, Brain Innovation B.V., Maastricht, the Netherlands) was used to extract and average the BOLD-signal values of all voxels in the individual neurofeedback target region at each TR. The resulting means were normalized to the range [0-10], in relation to the preceding baseline period (constituting a value of 0 ) and an individual maximum percent-signal change value, derived from the localizer run (constituting a value of 10 ). The resulting value range was binned into 10 segments and all segments of the thermometer display up to the given feedback value were greyed. Feedback was updated every 2 s.

\subsection{Post-hoc analysis of (f)MRI data}

\subsection{1 (f)MRI data pre-processing}

To answer the specific research questions of the current paper, offline analysis of the (f)MRI data was performed using BrainVoyager QX (v2.8, Brain Innovation, Maastricht, the Netherlands). Anatomical data sets were corrected for spatial intensity inhomogeneity. For all participants, the anatomical data set from the first session was transferred into ACPC space and the anatomical data set from the second session was automatically aligned to the ACPC version of the first data set. Both data sets were spatially normalized by Talairach transformation. All functional data sets underwent slice scan-time correction and temporal high-pass filtering 
(three cycles per time course). Three-dimensional (3D) head-motion detection and correction was applied by spatially aligning all functional volumes of a session to the first functional volume of the first run within that session. Finally, all functional runs were spatially normalized to Talairach space and interpolated to a 3- $\mathrm{mm}^{3}$ voxel resolution. For whole-brain and masked analysis, functional data was smoothed in 3D with a 4-mm FWHM Gaussian kernel.

\subsubsection{Extraction of striatum time-series}

One region of interest (ROIs) for the striatum was defined for each hemisphere based on peak coordinates from a recent meta-analysis on reward processing in fMRI (see Figure 2). For both hemispheres, selected coordinates marked the maximal spatial overlap of activation increases in response to reward of $126 \mathrm{fMRI}$ studies (Bartra et al., 2013). The MNI coordinates reported in the meta-analysis were converted into Talairach coordinates (Talairach \& Tournoux, 1988) using the Yale Biolmage Suite Package tal2mni tool (Lacadie, Fulbright, Rajeevan, Constable, \& Papademetris, 2008). Spherical volumes of interest (one left- and one righthemispheric) with a 3-mm radius were created around the particular coordinates. Both ROls were located in the anterior section of the corpus striatum, centered between caudate head and anterior putamen (left striatum: 123 voxels, $x=-14, y$ $=7, z=-2$, right striatum: 123 voxels, $x=-11, y=3, z=-3$; see Figure 2). The described approach increased the probability of detecting reward-related activation in the brain while not making a priori assumptions regarding anatomical sub-regions of the striatum contributing to the processing. For further analysis, the resulting striatum $\mathrm{ROI}$ in the hemisphere of the individual neurofeedback target region was chosen for each participant. As cortico-striatal structural connectivity is known to be dominantly ipsilateral (see for example Innocenti, Dyrby, Andersen, Rouiller, and Caminiti (2016), Jarbo and Verstynen (2015)) this approach increased 
the probability of detecting striatal activation specifically related to the cortical processes involved in the individual self-regulation task. For each ROI, eight time courses (from each of the four self-regulation runs per training condition) were extracted per participant.

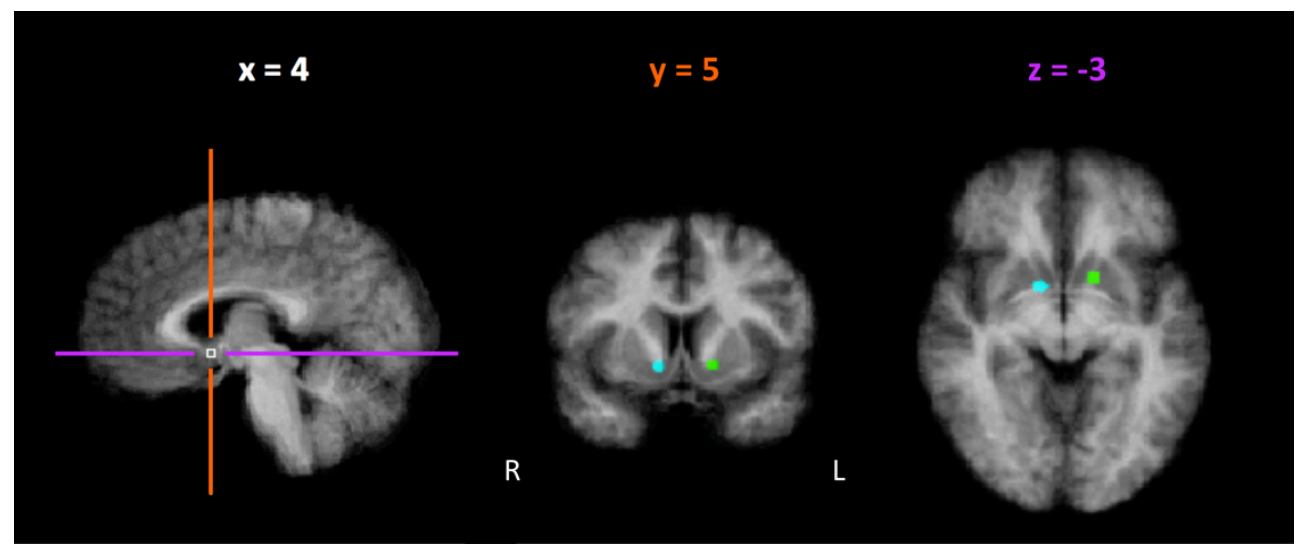

Figure 2: Definition of striatum regions of interest. The figure shows the right- and lefthemispheric striatum regions of interest $(R=$ right, $L=$ left $)$ overlaid on the mean of all individual anatomical data sets and slice positions of displayed coronal (orange) and axial (purple) slices. Regions of interests included all voxels in a 3-mm sphere centered around peak coordinates from a recent meta-analysis on reward processing representing maximal overlap of BOLD-signal increase in response to positive reward (Bartra et al., 2013). Provided coordinates are in Talairach space.

Moment-to-moment neurofeedback stimulation images (as presented to the participant during the neurofeedback experiment) were re-created applying the same procedure as described in Sorger et al. (2016) for the feedback sessions and the same procedure was applied post-hoc for no-feedback sessions, resulting in one picture per TR/scanned volume during modulation periods for both types of training. Using in-house software written in MATLAB (v8.1 R13; The MathWorks, Natick, USA), values of feedback magnitude were extracted from picture files. For each TR, one index of self-regulation accuracy was created by calculating the 
absolute difference between the target level and the feedback magnitude actually achieved by the participant. Subsequently, the time series of accuracy indices were convolved with a hemodynamic response function to create a self-regulation performance-predictor time course (representing the neurofeedback task accuracy at each TR across time) for subsequent correlation analysis with ROI time-courses.

\subsubsection{Statistical analysis}

\subsubsection{Whole-brain analysis}

To determine whether our sample showed activation during neurofeedback in coherence with recent meta-analytical evidence on neurofeedback, we analyzed whole-brain data in BrainVoyager QX by computing a group random-effects general linear model (GLM), including the types of training (feedback, no feedback), target levels (low: $30 \%$, medium: 60\%, high: $90 \%$ of the individual maxPSC), as well as six motion parameters as confounding predictors to estimate beta values. We employed a two-way within-subject design with target-level (low, medium and high) and type of training (no feedback and feedback) as factors. Subsequently, we compared activity increases during self-regulation with neurofeedback to passive viewing of neurofeedback was analysed (i.e. the resting condition) by contrasting activation across all target-level conditions during modulation periods with neurofeedback to baseline, during which participants passively observed fluctuations in the neurofeedback signal.

\subsubsection{Striatum region of interest analysis}

To determine whether striatum activation increases during rtfMRI neurofeedbackbased self-regulation compared to self-regulation without neurofeedback and whether this effect is influenced by the height of the desired target level, we performed a standard volume of interest analysis in BrainVoyager QX: Time-courses of all voxels within the meta-analytically defined striatum ROls were averaged to 
create one time-course of each functional run. By computing a group randomeffects general linear model (GLM) on the striatum ROI time-courses, including the HRF-convoluted predictors for types of training (feedback, no feedback), target levels (low: $30 \%$, medium: 60\%, high: $90 \%$ of the individual maxPSC), as well as six motion parameters as confounding predictors to estimate beta values. We employed a two-way within-subject design with target-level (low, medium and high) and type of training (no feedback and feedback) as factors. A two-way repeated measures analysis of variance (ANOVA, F-Test) with factors for target level and type of training was performed on the resulting striatum beta estimates.

\subsubsection{Time-resolved analysis of neurofeedback performance and striatal activation}

To investigate whether striatum activation during neurofeedback is modulated by the displayed information on self-regulation accuracy, performance-predictor time courses were correlated to striatum time courses. In order to also detect temporally delayed activation changes, the predictor time courses were shifted in time (see Figure 3). For all time-points during the modulation periods, predictor time courses were correlated to the striatum-ROI time courses within runs. One correlation coefficient was acquired separately for each temporal shift, with the maximum shift being seven TRs, resulting in eight correlation coefficients per run (including the correlation coefficient for the non-shifted time course).

Correlation coefficients were subsequently Fisher z-transformed and first averaged within subjects and conditions. To create stable estimates of the correlation between the two variables with expected high variability, resulting correlation means were temporally smoothed by averaging the z-transformed correlation means within two time-windows that were sufficiently distant in time to capture different BOLD response peaks: The early time window (0-3 TR shifts) included 
immediate BOLD changes with a margin for variability in BOLD timing and shape and delay in neural reactions. The late time window (4-7 TR shifts) included BOLD changes delayed for at least $8 \mathrm{~s}$ after an immediate BOLD response would be expected, so that BOLD changes in the late time window rather reflect a secondary stage of processing, as for example response preparation, not an immediate reaction to the rewarding feedback.

- High self-regulation accuracy

O Striatum activation peak

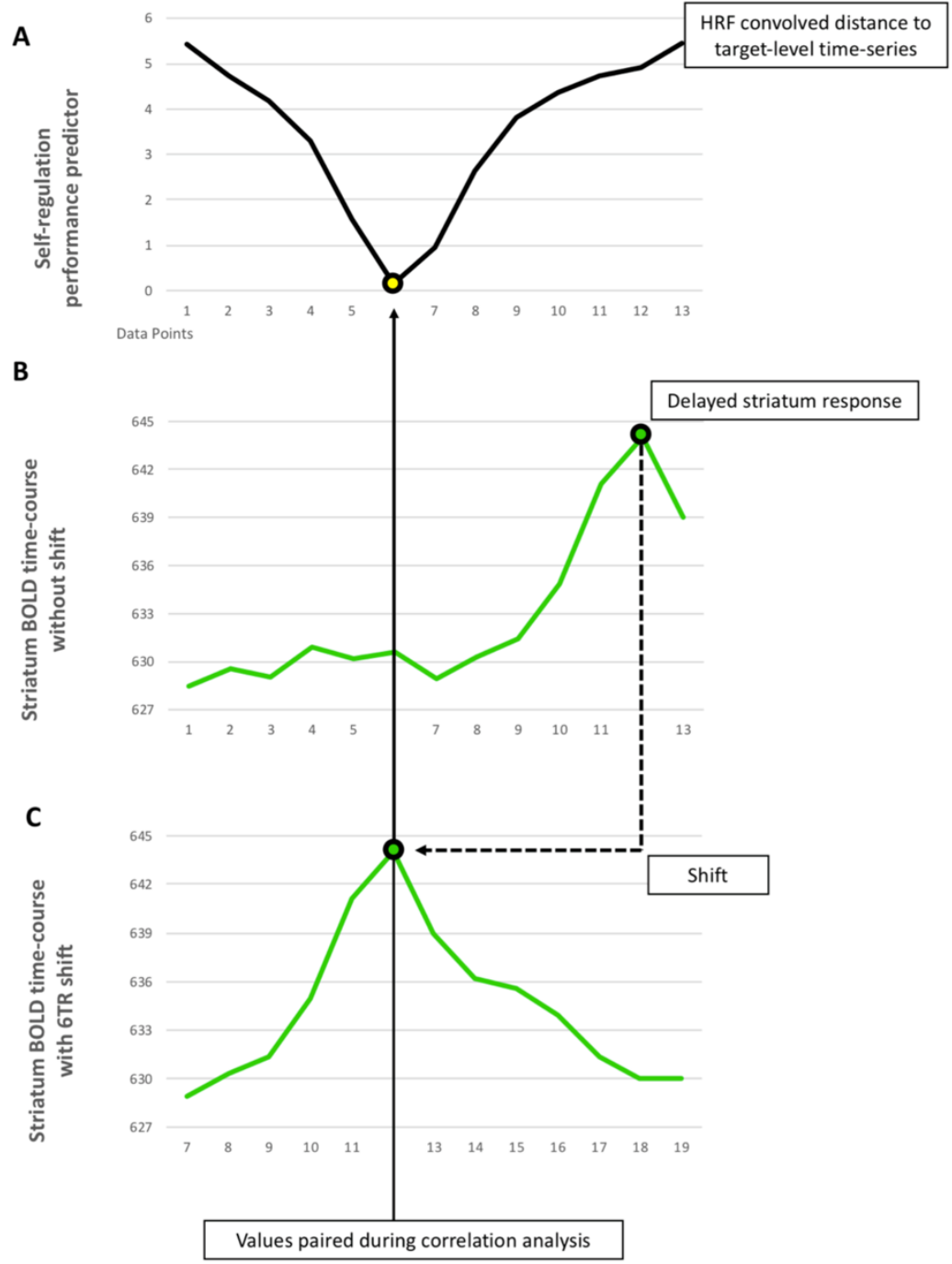


Figure 3: Time-resolved analysis of striatum activation in response to self-regulation success. The figure displays the logic of the performed correlation analysis. Simulated data during gradual self-regulation is shown: A. An HRF-convolved time series of performance indices is created from the absolute distance to the target activation level. Successful selfregulation (i.e., accurate regulation of the feedback signal to the target activation level) is represented by a low value. $B$. When a corresponding activation increase in the striatum ROI is delayed (in this example 6 TR), the activation peak is not paired to the improvement in performance during correlation analysis. C. Only, when the striatum time-course is shifted 6 TRs backwards, the increase in striatum activation is aligned to the decrease in absolute distance during correlation analysis.

This procedure resulted in one correlation coefficient per subject, time window and type of training (four correlation coefficients per participant). Z-transformed correlation coefficients were compared between types of training separately within the two time windows using student's paired-sample t-tests and applying Bonferroni correction for multiple comparison correction. Effect sizes were calculated based on Cohens D (Cohen, 1988) adapted for paired measures (Morris \& DeShon, 2002).

\subsubsection{Masked voxel-wise analysis of the neurofeedback network}

To identify activation increases during neurofeedback independent of selfregulation across the whole neurofeedback network, the voxel-wise group randomeffects GLM was restricted to a mask constituting of voxels within $15 \mathrm{~mm}$ radii around cortical and $20 \mathrm{~mm}$ radii around the two subcortical meta-analytic peak voxels centred in the thalamus (with the resulting mask also including the basal ganglia), that marked activation increases across several neurofeedback studies compared to rest, using the peak voxels described by Emmert et al. (2016) in the PACC, aINS, VIPFC, dIPFC, temporo-parietal and occipital cortex and two subcortical peak coordinates that constituted local maxima of several subcortical substructures 
(putamen, caudate, nucleus accumbens, globus pallidus, thalamus). The coordinates were transferred into Talairach space using the Yale Biolmage Suite Package tal2mni tool (Lacadie et al., 2008). Differences between the two training conditions were compared by contrasting activation during modulation periods with neurofeedback to modulation periods without neurofeedback across the three target levels. Results were cluster corrected using Monte-Carlo simulations with 1000 iterations, a FWHM of 1,608 with an initial threshold of $p<0.01$. Additionally, a liberal correction threshold was applied deliberately for decreasing the likelihood of missing potentially lower / more scattered activation in prefrontal control areas.

\section{Results}

\subsection{Effect of self-regulation}

The contrast for self-regulation with neurofeedback compared to passive viewing of neurofeedback (i.e. rest) revealed an extensive network of regional increases (FDR corrected, $\mathrm{q}<0.05$ ), encompassing the bilateral precentral gyrus, the bilateral anterior insula, bilateral visual cortices, bilateral dorsolateral prefrontal cortex (dIPFC), left ventrolateral prefrontal cortex (VIPFC), bilateral supplementary motor area (SMA), bilateral posterior anterior cingulate cortex (pACC), left frontopolar cortex and an extensive subcortical cluster encompassing the striatum, thalamus and claustrum and deactivation (Figure 4) across the bilateral default mode network (transverse temporal gyrus, angular gyrus (AG), precuneus, medial prefrontal cortex (mPFC)) and the posterior insula (pINS) bilaterally (Table 1). No significant differences between target levels or interactions were observed. 

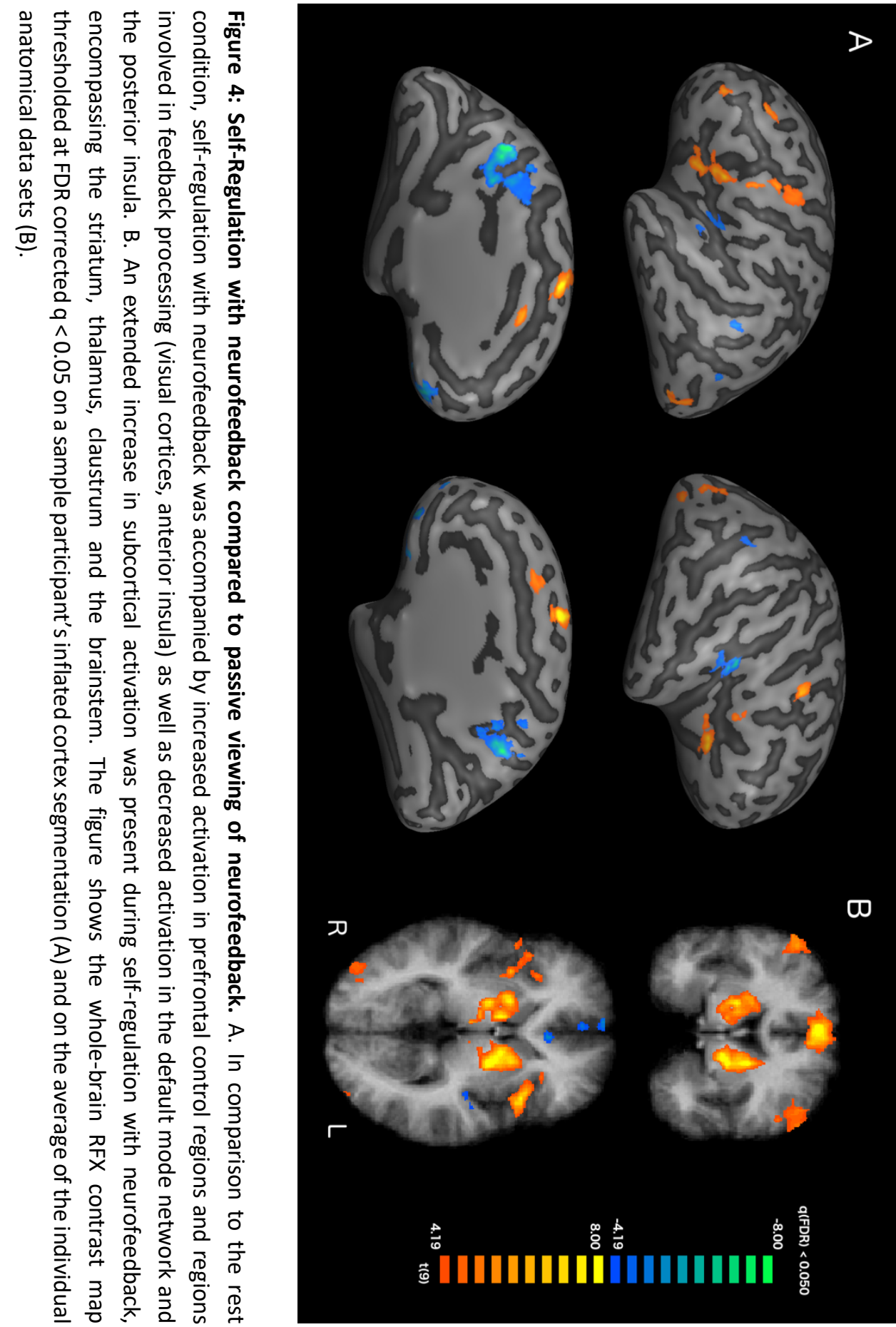


\begin{tabular}{|c|c|c|c|c|c|}
\hline Increased activation & \multicolumn{3}{|c|}{ Peak Voxel Coordinates } & \multicolumn{2}{|c|}{ Peak voxel statistics } \\
\hline Visual feedback and reward & $\mathbf{x}$ & $\mathbf{y}$ & $\mathbf{z}$ & $\mathbf{t}$ & $\mathbf{p}$ \\
\hline Subcortical L / anterior Insula L & -18 & -4 & 4 & 12.3283 & 0.000001 \\
\hline Anterior insula $\mathrm{R}$ & 33 & 20 & 10 & 10.0164 & 0.000004 \\
\hline Subcortical R & 18 & 8 & 16 & 9.4796 & 0.000006 \\
\hline Visual cortex L & -39 & -82 & -9 & 6.2985 & 0.000141 \\
\hline Visual cortex $\mathrm{R}$ & 36 & -85 & 7 & 5.8471 & 0.000245 \\
\hline \multicolumn{6}{|l|}{ Self-regulation and attention } \\
\hline Supplementary motor area bilateral & 3 & -1 & 58 & 13.0617 & 0.000001 \\
\hline Ventrolateral PFC / Precentral gyrus L & -60 & 11 & 16 & 8.7361 & 0.000011 \\
\hline Dorsal anterior cingulate bilateral & 0 & 14 & 43 & 7.7295 & 0.000029 \\
\hline Precentral gyrus L & -39 & 29 & 28 & 7.2432 & 0.000049 \\
\hline Precentral Gyrus R & 51 & 2 & 46 & 7.2152 & 0.000055 \\
\hline Frontopolar / dorsolateral PFC L & -45 & 48 & 13 & 7.0833 & 0.000058 \\
\hline \multicolumn{6}{|l|}{ Decreased activation } \\
\hline Precuneus & -3 & -55 & 31 & -10.6369 & 0.000002 \\
\hline Posterior insula $\mathrm{R}$ & 39 & -22 & 19 & -9.4743 & 0.000006 \\
\hline Posterior insula L & -51 & -22 & 13 & -8.4177 & 0.000015 \\
\hline Medial PFC & 0 & 41 & -9 & -7.5212 & 0.000036 \\
\hline Posterior temoral cortex/ Angular gyrus L & -60 & -58 & 23 & -6.7828 & 0.000081 \\
\hline Posterior temoral cortex/ Angular gyrus R & 48 & -61 & 25 & -6.7624 & 0.000082 \\
\hline
\end{tabular}

Table 1: Self-Regulation with neurofeedback compared to passive viewing of neurofeedback. Self-regulation with neurofeedback was accompanied by increased activation in prefrontal control regions (dorsolateral and ventrolateral PFC, dorsal anterior cingulate, precentral gyrus, supplementary motor area) and regions involved in feedback processing (visual cortices, anterior insula and an extended subcortical cluster) as well as decreased activation in the default mode network and the posterior insula. The table contains coordinates and statistics of peak voxels for the whole-brain RFX contrast map thresholded at FDR corrected $q<0.05$ (coordinates in Talairach space). Remarks: PFC, Prefrontal cortex; $L$, left; $R$, right. 


\subsection{Effect of neurofeedback information on striatum activation}

The main effect for type of training was significant ( $p=0.036$, one-sided, Figure $5 A)$ but there was no main effect of target level ( $p=0.14$, one-sided) and no significant interaction $(p=0.08$, one-sided). Correspondingly, eight out of ten participants showed increased mean beta values during self-regulation with neurofeedback compared to self-regulation without neurofeedback but striatum activation did not differ in a consistent fashion between target levels across participants (see Figure $5 B)$.

A
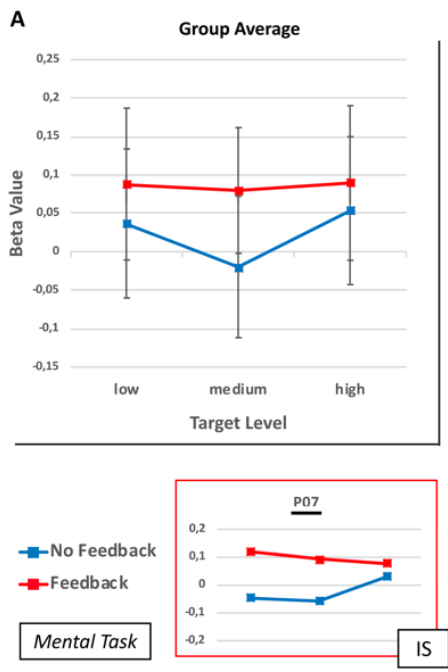

B
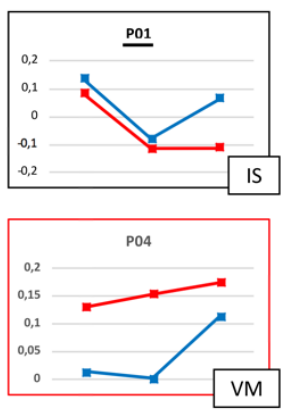

$\underline{\mathrm{P} 08}$

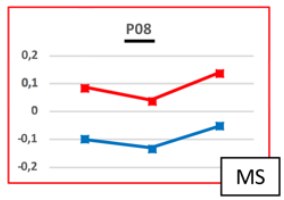

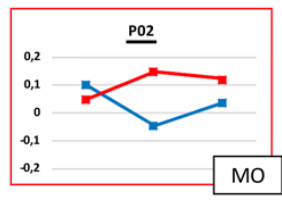
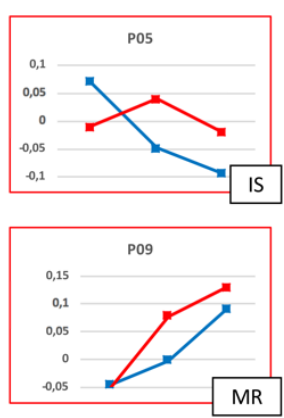
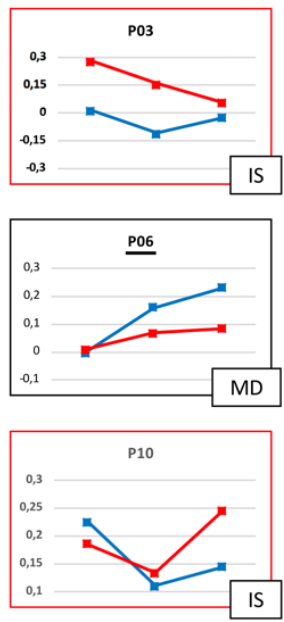

Figure 5: Effect of gradual self-regulation success on striatum activation (group and singlesubject results). The figure visualizes the BOLD-signal level within the striatum region of interest ipsilateral to the neurofeedback target region for the two type-of-training conditions and across the different target-level conditions: A. Mean beta values for each target-level condition across all participants separately for the no-feedback (blue) and feedback (red) condition. Error bars represent standard errors of the means. When pooling the data across the target-level conditions, the difference of mean-beta values for the two type-of training conditions (feedback, no-feedback) was significant ( $p<0.05$, Bonferronicorrected, one-sided) B. Single-subject mean beta values separately for each target-level and type-of-training condition. In $80 \%$ of participants (red-rimmed), the mean striatum activation (i.e., pooled activation across the three target-level conditions) was higher in the feedback compared to the no-feedback condition. Remark. Participants with black 
underline underwent the feedback condition first and no-feedback condition second. Abbreviations for mental strategies: IS = Inner speech, $\mathrm{MO}=$ mental orchestra, $\mathrm{VM}=$ visual motion imagery, $\mathrm{MD}=$ mental drawing, $\mathrm{MS}=$ mental sounds, $\mathrm{MR}=$ mental running.

\subsection{Modulation of striatum activation by self-regulation success}

An extensive analysis of task performance in the given sample can be found in Sorger et al. (2016). In short, participants were able to increase the BOLD signal magnitude to target levels in a gradual fashion across both training conditions (no-

feedback and feedback), but most participants demonstrated slightly increased ability to differentiate between target levels when provided with neurofeedback information. Both training condition were matched closely with regard to the absolute distance to the desired target level (absolute distance mean (feedback) = $3.866, \operatorname{SEM}=0.19 ;$ mean $($ no feedback) $=3.858, \operatorname{SEM}=0.22 ; \mathrm{p}=0.96$ ).

Correlation analysis between performance-predictor time courses and the striatum time courses resulted in one mean z-transformed correlation coefficients per typeof-training condition (feedback, no feedback) and the two predefined time windows (early, late): Early time window: Neurofeedback- $r_{z}=0.047, S E M=0.01$; No-feedback- $r_{z}=-0.018$, SEM $=0.02$; Late time window: Neurofeedback $-r_{z}=-0.065$, SEM $=0.03$; No-feedback mean $r_{z}=0.017$, SEM $=0.02$ (Figure 6A). Subsequent paired t-tests between training types indicated a significant difference between correlation coefficients only for the late time window ( $p=0.044$ (Bonferroni corrected), Cohens $d=0.912$ ) but not for the early time window ( $p=0.13$ (Bonferroni corrected), Cohens $d=-0.677$ ). The effect was consistent across participants (see Figure 6B): 8 out of 10 participants showed a more negative mean correlation during gradual self-regulation with feedback compared to gradual selfregulation without feedback in this time window. 


\subsection{Sub-components of the neurofeedback network involved in feedback processing}

Voxel-wise analysis restricted to regions showing increased activation during neurofeedback (as defined based on meta analytic coordinates from Emmert et al. (2016)) revealed no significant differences between self-regulation with neurofeedback compared to self-regulation without neurofeedback (FDR corrected, $\mathrm{q}<0.05)$. Deliberately applying a liberal correction threshold for decreasing the likelihood of false negatives in the our small sample revealed activation differences in the left anterior striatum, right anterior insula and left visual cortices and lower activation in the bilateral posterior striatum / thalamus remained, cluster corrected using Monte Carlo simulations with an initial threshold of $p<0.01$.

A

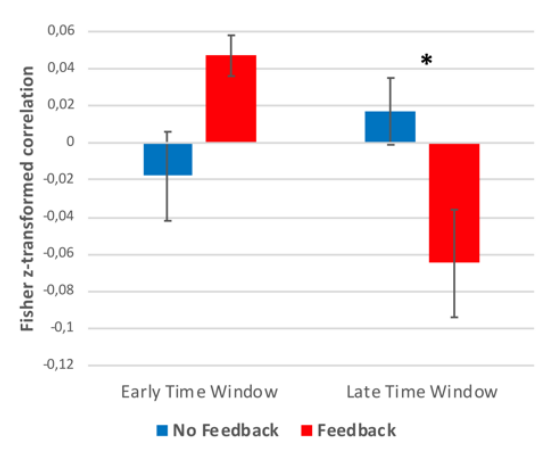

B

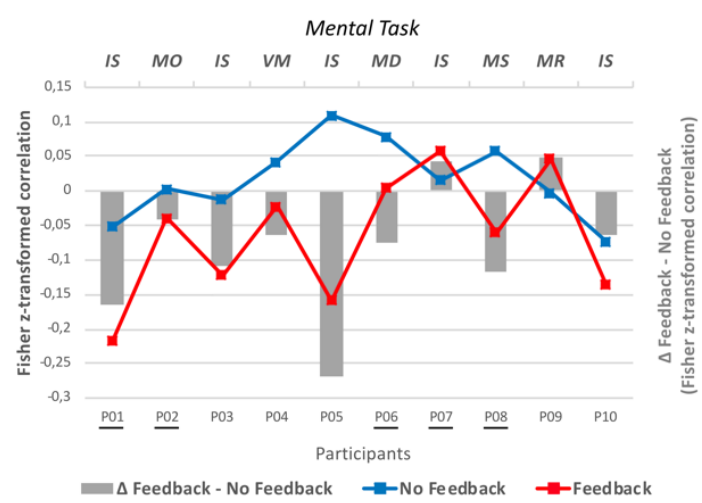

Figure 6: Relationship between self-regulation success and striatum activation level (group and single subject results). Relationship between absolute distance to target activation level and striatum activation separately for the two type-of-training conditions. A. Mean Fisher z-transformed correlation coefficients between self-regulation success and striatum activation separately for an early time window (0-3 TR shift, immediate and slightly delayed striatum activation) and a late time window (4-7 TR shift, delayed striatum activation). The difference of the correlation values with respect to the two type-of-training conditions (feedback, no feedback) was only significant for the late time window $(p<0.05$, Bonferroni-corrected, one-sided). B. Single-subject results for the late time window. $80 \%$ of 
participants showed a more negative correlation between distance to target-level and striatum activation during gradual self-regulation when receiving neurofeedback. Remark. Participants with black underline underwent feedback condition first and no-feedback condition seconds. Abbreviations for mental tasks: IS = Inner speech, $\mathrm{MO}=$ mental orchestra, $\mathrm{VM}=$ visual motion imagery, $\mathrm{MD}=$ mental drawing, $\mathrm{MS}=$ mental sounds, $\mathrm{MR}=$ mental running.

\section{Discussion}

\subsection{Brain activation in response to neurofeedback during gradual self-regulation}

The main aim of this study was to identify activation related to neurofeedback processing during neurofeedback-guided self-regulation. We investigated this research question through analysis at whole-brain level, in the striatum, a key region implicated in feedback and reward processing, and within a whole network of regions that reliably shows increased activation during neurofeedback as identified by a recent meta-analysis.

We could replicate recent meta-analytical findings (Emmert et al., 2016) within a single sample with regard to joint activation of cognitive control areas and areas involved in feedback learning by observing extended activation increases in prefrontal control hubs ( $\mathrm{PACC}$, lateral and posterior PFC) as well as regions involved in feedback and reward processing (aINS, striatum, visual cortices), the thalamus and deactivation in the default network across different mental tasks during neurofeedback. Region of interest analysis focussed on the striatum revealed significantly higher activation during gradual self-regulation with than without feedback, suggesting that during neurofeedback, the observed striatum 
modulations reflect feedback learning and not self-regulation per se, as participants achieved successful self-regulation already without receiving feedback (for an extensive discussion of self-regulation in the sample see Sorger et al. (2016)) and both self-regulation conditions did not differ with regard to the provided visual markers of task performance, i.e. absolute distance to target level. As participants were engaged in different self-regulation task domains, the observed increase in activation was not related to a specific task domain, but specifically driven by neurofeedback. Subsequent analysis on the relationship between visual information provided during neurofeedback and striatum activation showed that more accurate neurofeedback performance was accompanied by an increased BOLD-signal level in the anterior striatum in a late time window (8-14s after a particular neurofeedback value was visually displayed), suggesting that the observed striatal activation increases during neurofeedback are indeed reflecting the processing of feedback information. While region of interest analysis revealed increased activation during neurofeedback compared to self-regulation without neurofeedback in the anterior striatum, we failed to detect activation differences during voxel-wise analysis within the network of regions commonly involved in neurofeedback (Emmert et al., 2016). As we cannot exclude the absence of activation differences within other regions of the network (especially in feedback processing regions and visual areas as suggested by liberal cluster corrected analysis), further research with higher statistical power is needed to describe the distribution of activation within the whole network in comparison to self-regulation without neurofeedback, as the sample size of the given study constituted a limitation with regard to statistical power, as well as a potentially slight variance introduced by different MR systems. 
Overall, our findings are in line with recent theoretical approaches that suggest different sub-components of the neurofeedback network for feedback processing and self-regulation (Sitaram et al., 2017). While the anterior striatum appears to serve an unique function in response to neurofeedback, especially the lateral PFC and the ACC (of the network activated during neurofeedback in this study) have been defined as key regions in cognitive control in general (MacDonald, Cohen, Stenger, \& Carter, 2000). Both regions are also jointly activated during various task modalities that involve cognitive control, including emotion regulation (Etkin, Egner, \& Kalisch, 2011; Goldin, McRae, Ramel, \& Gross, 2008), response inhibition (Cai et al., 2015) and attentional control (Weissman, Gopalakrishnan, Hazlett, \& Woldorff, 2004), supporting their role as the general basis of self-regulation. Fittingly, participants were instructed to dynamically engaged and disengage from their mental task during self-regulation (indeed participants confirmed to have followed these general strategies closely, see Sorger et al. (2016)), thereby shifting their focus of attention to and away from the mental content driving the feedback, modulating activation in their attentional system during self-regulation as in accordance with the observed prefrontal and parietal activation.

Conforming to the observed modulations of striatum activation by neurofeedback, most theoretical approaches on neurofeedback underline the importance of reward processing during neurofeedback, although several different working mechanisms have been proposed (for a recent overview see Sitaram et al. (2017)). As the parametric activation paradigm applied in this study differs from previous research regarding the possibility to receive rewarding neurofeedback by up- as well as by down-regulating activation adequately in relation to the target level, it remains open whether graded neurofeedback, as employed here, recruits the striatum differently compared to neurofeedback studies aiming at maximizing the 
neurofeedback magnitude, as task load and reward probability are known to modulate activation strength and timing of reward system activation (Cardinal, 2006; Stalnaker, Calhoon, Ogawa, Roesch, \& Schoenbaum, 2012). Additionally, it would be important for further research to examine strategically whether modulating the reward contained in neurofeedback can be used to optimize its influence on the striatum, for example by investigating the effect of monetary reward for performance. Furthermore, to ensure that participants could optimally perform gradual self-regulation in both conditions, and focus on the relevant marker of performance (i.e. either derived from neurofeedback or in the no-

feedback condition from introspection), no blinding was applied in the current study. As the lack of (double) blinding constitutes a limitation of our design, future research should investigate the effects of blinding on the reward system during selfregulation with neurofeedback.

\subsection{The influence of neurofeedback on different stages of reward processing}

We also investigated how neurofeedback influences different stages of reward processing. In the current study, reward values were assigned to the distance between the instructed target activation level and the achieved activation level, which was updated every 2 s. Interestingly, the observed neurofeedback effect was substantially delayed (a significant difference between both training conditions was observed only in a time window 8-14s after a corresponding feedback information was provided). Taking into account that rtfMRI-based neurofeedback is delayed over several seconds, the reward information provided during and shortly after a mental action is unrelated to the neural activity subserving the mental action itself. It remains to be determined if reception of conflicting reward information during 
performance of a mental action leads to alterations in neurofeedback processing, as besides attributing value to a certain stimulus, the striatum also detects relations between performed actions and rewards (FitzGerald, Schwartenbeck, \& Dolan, 2014; Haruno et al., 2004; Kim, Sul, Huh, Lee, \& Jung, 2009) and predicts when a reward should occur (Kohrs et al., 2012). Additionally to receiving noncorresponding feedback during or shortly after a mental action, the predictability of reward information is also reduced due to noise or other confounding factors that distort the neurofeedback signal. Violations of reward expectancies as well as uncertainty of receiving rewards lead to alterations in striatum activation (Kohrs et al., 2012; McClure et al., 2003; Pagnoni et al., 2002). Both uncertainty and conflicting reward information could contribute to the difficulty to detect an immediate neurofeedback response in an early time window after the feedback is presented. Focusing on creating more direct closed-loop approaches (El Hady, 2016; Potter, El Hady, \& Fetz, 2014), e.g., by neurofeedback-guided brain stimulation systems that stimulate the striatum directly, could help to detect optimal time windows for operant conditioning and increase the efficacy of neurofeedback strongly.

With respect to the interpretability of the current results regarding different phases of reward processing, it has also to be noted that different stages of feedback processing do not only differ in time, but also recruit different sub-regions of the striatum (Balleine et al., 2007; Sleezer \& Hayden, 2016; Tanaka et al., 2004). As the current study aimed at describing the temporal relationship between visual neurofeedback information and striatum activation for the first time, regions of interest were chosen based on meta-analytic peak coordinates on reward processing in fMRI, to increase the probability of detecting reward related activation. Due to this region-of-interest selection approach, different anatomical 
sub-regions of the anterior striatum contribute to the observed activation, and as a consequence we cannot make any strong claims regarding the exact anatomical sub-structures underlying this activation pattern.

However, the ventral section of putamen and caudate indeed have been shown to create reward predictions using temporal information (Hiebert et al., 2014) supporting the interpretation that here temporal properties of reward information are crucial. Especially anterior caudate has also been associated with biasing actions based on reward information (O'Doherty et al., 2004; Tanaka, Balleine, \& O'Doherty, 2008; Tricomi, Delgado, \& Fiez, 2004; Valentin, Dickinson, \& O'Doherty, 2007), suggesting that the ROI signal represents merged processes of reward prediction and action selection, thereby reflecting the interwoven transitions between different reward processing stages in the striatum (Haber et al., 2000).

\subsection{Linking mental actions to the information contained in neurofeedback}

Neurofeedback differs from other domains of feedback learning during the learning process in that actions driving the reward are purely mental actions. To understand which mental actions lead to rewards, participants rely on introspection. The conscious monitoring of mental actions requires meta-awareness, which recruits a distributed network of areas, including the anterior cingulate cortex and the insula (Schooler et al., 2011). In a loop-like fashion, these hubs have been suggested to interact with other higher-order cognitive networks involved in working memory and attention, the striatum, the thalamus and regions recruited by the specific selfregulation task during neurofeedback (Emmert et al., 2017; McCaig, Dixon, Keramatian, Liu, \& Christoff, 2011; Sitaram et al., 2017). Neural-feedback loops 
between these networks and the striatum could be crucial in identifying a relationship between mental actions and corresponding reward values. The complex pattern of continuous top-down input from other regions to the striatum during reward processing (Haruno \& Kawato, 2006), could be an important contributing factor for the observed delay in striatum reactivity to neurofeedback.

However, action-effect mapping is not selectively dependent on conscious introspection (Hommel, 1996). Accordingly, recent studies (Ramot, Grossman, Friedman, \& Malach, 2016) demonstrated that covert neurofeedback, i.e., during which participants are not aware of the fact that they received neurofeedback, was accompanied by increased striatum activation (Ramot et al., 2016). Both automatic reward processing as well as conscious self-regulation have been argued to be crucial in neurofeedback (Sitaram et al., 2017) but a mechanistic model of how automatic and subsequent stages of reward processing interact during neurofeedback is still lacking. For future research to tackle this issue, the temporal properties of neurofeedback should be taken into account because action-reward mapping is known to be strongly influenced by the delay of a reward (Dobryakova \& Tricomi, 2013; Tanaka et al., 2004).

\subsection{Conclusion}

This study demonstrates that neurofeedback contributes to self-regulation through regions involved in feedback and reward processing, which share activation between different mental tasks. Focussing on the striatum as a key region in reward processing, we demonstrated increased activation in the anterior striatum during self-regulation with neurofeedback, which correlated with self-regulation success. The substantial delay in the observed effect suggests that these modulations reflect 
later stages of reward processing beyond simple detection of external rewards, but further research is needed to understand the mechanisms of neurofeedback reward learning. As trained associations between actions and rewards (for example during operant conditioning) are key to learning, the given results provide a promising outlook for neurofeedback to facilitate learning with the potential for operant conditioning of mental actions. 


\section{References}

Amano, K., Shibata, K., Kawato, M., Sasaki, Y., \& Watanabe, T. (2016). Learning to associate orientation with color in early visual areas by associative decoded fMRI neurofeedback. Current Biology, 26(14), 1861-1866.

Balleine, B. W., Delgado, M. R., \& Hikosaka, O. (2007). The role of the dorsal striatum in reward and decision-making. Journal of Neuroscience, 27(31), 81618165.

Bartra, O., McGuire, J. T., \& Kable, J. W. (2013). The valuation system: a coordinatebased meta-analysis of BOLD fMRI experiments examining neural correlates of subjective value. Neurolmage, 76, 412-427.

Birbaumer, N., Ruiz, S., \& Sitaram, R. (2013). Learned regulation of brain metabolism. Trends in cognitive sciences, 17(6), 295-302.

Boynton, G. M., Engel, S. A., Glover, G. H., \& Heeger, D. J. (1996). Linear systems analysis of functional magnetic resonance imaging in human V1. Journal of Neuroscience, 16(13), 4207-4221.

Cai, W., Chen, T., Ryali, S., Kochalka, J., Li, C.-S. R., \& Menon, V. (2015). Causal interactions within a frontal-cingulate-parietal network during cognitive control: Convergent evidence from a multisite-multitask investigation. Cerebral Cortex, 26(5), 2140-2153.

Cardinal, R. N. (2006). Neural systems implicated in delayed and probabilistic reinforcement. Neural Networks, 19(8), 1277-1301.

Caria, A., Veit, R., Sitaram, R., Lotze, M., Weiskopf, N., Grodd, W., \& Birbaumer, N. (2007). Regulation of anterior insular cortex activity using real-time fMRI. Neurolmage, 35(3), 1238-1246.

Cohen, J. (1988). Statistical power analysis for the behavioral sciences 2nd edn: Erlbaum Associates, Hillsdale.

Davelaar, E. J. (2018). Mechanisms of neurofeedback: A computation-theoretic 
approach. Neuroscience, 378, 175-188.

DePasque Swanson, S., \& Tricomi, E. (2014). Goals and task difficulty expectations modulate striatal responses to feedback. Cognitive, Affective, \& Behavioral Neuroscience, 14(2), 610-620.

Dobryakova, E., \& Tricomi, E. (2013). Basal ganglia engagement during feedback processing after a substantial delay. Cognitive, Affective, \& Behavioral Neuroscience, 13(4), 725-736.

El Hady, A. (2016). Closed loop neuroscience: Academic Press.

Emmert, K., Kopel, R., Koush, Y., Maire, R., Senn, P., Van De Ville, D., \& Haller, S. (2017). Continuous vs. intermittent neurofeedback to regulate auditory cortex activity of tinnitus patients using real-time fMRI-A pilot study. Neurolmage: Clinical, 14, 97-104.

Emmert, K., Kopel, R., Sulzer, J., Brühl, A. B., Berman, B. D., Linden, D. E., . . Frank, S. (2016). Meta-analysis of real-time fMRI neurofeedback studies using individual participant data: How is brain regulation mediated? Neurolmage, $124,806-812$.

Etkin, A., Egner, T., \& Kalisch, R. (2011). Emotional processing in anterior cingulate and medial prefrontal cortex. Trends in cognitive sciences, 15(2), 85-93.

Fetz, E. E. (2007). Volitional control of neural activity: implications for braincomputer interfaces. The Journal of physiology, 579(3), 571-579.

FitzGerald, T. H., Schwartenbeck, P., \& Dolan, R. J. (2014). Reward-related activity in ventral striatum is action contingent and modulated by behavioral relevance. Journal of Neuroscience, 34(4), 1271-1279.

Gevensleben, H., Holl, B., Albrecht, B., Schlamp, D., Kratz, O., Studer, P., .. . Heinrich, H. (2009). Distinct EEG effects related to neurofeedback training in children with ADHD: a randomized controlled trial. International Journal of Psychophysiology, 74(2), 149-157. 
Goldin, P. R., McRae, K., Ramel, W., \& Gross, J. J. (2008). The neural bases of emotion regulation: reappraisal and suppression of negative emotion. Biological psychiatry, 63(6), 577-586.

Grech, R., Cassar, T., Muscat, J., Camilleri, K. P., Fabri, S. G., Zervakis, M., . . . Vanrumste, B. (2008). Review on solving the inverse problem in EEG source analysis. Journal of neuroengineering and rehabilitation, 5(1), 25.

Gustavo, B., Soares, S., \& Paton, J. J. (2015). A scalable population code for time in the striatum. Current Biology, 25(9), 1113-1122.

Haber, S. N., Fudge, J. L., \& McFarland, N. R. (2000). Striatonigrostriatal pathways in primates form an ascending spiral from the shell to the dorsolateral striatum. Journal of Neuroscience, 20(6), 2369-2382.

Haller, S., Kopel, R., Jhooti, P., Haas, T., Scharnowski, F., Lovblad, K.-O., . . . Van De Ville, D. (2013). Dynamic reconfiguration of human brain functional networks through neurofeedback. Neurolmage, 81, 243-252.

Hamilton, J. P., Glover, G. H., Hsu, J. J., Johnson, R. F., \& Gotlib, I. H. (2011). Modulation of subgenual anterior cingulate cortex activity with real-time neurofeedback. Human Brain Mapping, 32(1), 22-31.

Haruno, M., \& Kawato, M. (2006). Heterarchical reinforcement-learning model for integration of multiple cortico-striatal loops: fMRI examination in stimulusaction-reward association learning. Neural Networks, 19(8), 1242-1254.

Haruno, M., Kuroda, T., Doya, K., Toyama, K., Kimura, M., Samejima, K., . . Kawato, M. (2004). A neural correlate of reward-based behavioral learning in caudate nucleus: a functional magnetic resonance imaging study of a stochastic decision task. Journal of Neuroscience, 24(7), 1660-1665.

Hiebert, N. M., Vo, A., Hampshire, A., Owen, A. M., Seergobin, K. N., \& MacDonald, P. A. (2014). Striatum in stimulus-response learning via feedback and in decision making. Neurolmage, 101, 448-457. 
Hommel, B. (1996). The cognitive representation of action: Automatic integration of perceived action effects. Psychological research, 59(3), 176-186.

Innocenti, G. M., Dyrby, T. B., Andersen, K. W., Rouiller, E. M., \& Caminiti, R. (2016). The crossed projection to the striatum in two species of monkey and in humans: behavioral and evolutionary significance. Cerebral Cortex, bhw161.

Jarbo, K., \& Verstynen, T. D. (2015). Converging structural and functional connectivity of orbitofrontal, dorsolateral prefrontal, and posterior parietal cortex in the human striatum. Journal of Neuroscience, 35(9), 3865-3878.

Johnston, S. J., Boehm, S. G., Healy, D., Goebel, R., \& Linden, D. E. (2010). Neurofeedback: A promising tool for the self-regulation of emotion networks. Neurolmage, 49(1), 1066-1072.

Jung, W. H., Jang, J. H., Park, J. W., Kim, E., Goo, E.-H., Im, O.-S., \& Kwon, J. S. (2014). Unravelling the intrinsic functional organization of the human striatum: a parcellation and connectivity study based on resting-state fMRI. PloS one, 9(9), e106768.

Kim, H., Sul, J. H., Huh, N., Lee, D., \& Jung, M. W. (2009). Role of striatum in updating values of chosen actions. Journal of Neuroscience, 29(47), 14701-14712.

Kjaer, T. W., Bertelsen, C., Piccini, P., Brooks, D., Alving, J., \& Lou, H. C. (2002). Increased dopamine tone during meditation-induced change of consciousness. Cognitive Brain Research, 13(2), 255-259.

Kohrs, C., Angenstein, N., Scheich, H., \& Brechmann, A. (2012). Human striatum is differentially activated by delayed, omitted, and immediate registering feedback. Frontiers in human neuroscience, 6 .

Lacadie, C. M., Fulbright, R. K., Rajeevan, N., Constable, R. T., \& Papademetris, X. (2008). More accurate Talairach coordinates for neuroimaging using nonlinear registration. Neurolmage, 42(2), 717-725. 
Lacroix, J. M., \& Gowen, A. H. (1981). The acquisition of autonomic control through biofeedback: some tests of discrimination theory. Psychophysiology, 18(5), 559-572.

MacDonald, A. W., Cohen, J. D., Stenger, V. A., \& Carter, C. S. (2000). Dissociating the role of the dorsolateral prefrontal and anterior cingulate cortex in cognitive control. Science, 288(5472), 1835-1838.

Marchesotti, S., Martuzzi, R., Schurger, A., Blefari, M. L., del Millán, J. R., Bleuler, H., \& Blanke, O. (2017). Cortical and subcortical mechanisms of brain-machine interfaces. Human Brain Mapping, 38(6), 2971-2989.

McCaig, R. G., Dixon, M., Keramatian, K., Liu, I., \& Christoff, K. (2011). Improved modulation of rostrolateral prefrontal cortex using real-time fMRI training and meta-cognitive awareness. Neurolmage, 55(3), 1298-1305.

McClure, S. M., Berns, G. S., \& Montague, P. R. (2003). Temporal prediction errors in a passive learning task activate human striatum. Neuron, 38(2), 339-346.

Megumi, F., Yamashita, A., Kawato, M., \& Imamizu, H. (2015). Functional MRI neurofeedback training on connectivity between two regions induces longlasting changes in intrinsic functional network. Frontiers in human neuroscience, 9.

Morris, S. B., \& DeShon, R. P. (2002). Combining effect size estimates in metaanalysis with repeated measures and independent-groups designs. Psychological methods, 7(1), 105.

Mottaz, A., Solcà, M., Magnin, C., Corbet, T., Schnider, A., \& Guggisberg, A. G. (2015). Neurofeedback training of alpha-band coherence enhances motor performance. Clinical Neurophysiology, 126(9), 1754-1760.

O'Doherty, J., Dayan, P., Schultz, J., Deichmann, R., Friston, K., \& Dolan, R. J. (2004). Dissociable Roles of Ventral and Dorsal Striatum in Instrumental Conditioning. Science, 304(5669), 452. 
Pagnoni, G., Zink, C. F., Montague, P. R., \& Berns, G. S. (2002). Activity in human ventral striatum locked to errors of reward prediction. Nature neuroscience, 5(2), 97.

Potter, S. M., El Hady, A., \& Fetz, E. E. (2014). Closed-loop neuroscience and neuroengineering. Frontiers in neural circuits, 8, 115.

Ramot, M., Grossman, S., Friedman, D., \& Malach, R. (2016). Covert neurofeedback without awareness shapes cortical network spontaneous connectivity. Proceedings of the National Academy of Sciences, 113(17), E2413-E2420.

Robbins, T. W., \& Everitt, B. J. (1996). Neurobehavioural mechanisms of reward and motivation. Current opinion in neurobiology, 6(2), 228-236.

Samejima, K., Ueda, Y., Doya, K., \& Kimura, M. (2005). Representation of ActionSpecific Reward Values in the Striatum. Science, 310(5752), 1337-1340.

Scheinost, D., Stoica, T., Saksa, J., Papademetris, X., Constable, R., Pittenger, C., \& Hampson, M. (2013). Orbitofrontal cortex neurofeedback produces lasting changes in contamination anxiety and resting-state connectivity. Translational psychiatry, 3(4), e250.

Schooler, J. W., Smallwood, J., Christoff, K., Handy, T. C., Reichle, E. D., \& Sayette, M. A. (2011). Meta-awareness, perceptual decoupling and the wandering mind. Trends in cognitive sciences, 15(7), 319-326.

Sitaram, R., Ros, T., Stoeckel, L., Haller, S., Scharnowski, F., Lewis-Peacock, J., . . . Oblak, E. (2017). Closed-loop brain training: the science of neurofeedback. Nature reviews neuroscience, 18(2), 86.

Sleezer, B. J., \& Hayden, B. Y. (2016). Differential contributions of ventral and dorsal striatum to early and late phases of cognitive set reconfiguration. Journal of cognitive neuroscience.

Sorger, B., Kamp, T., Weiskopf, N., Peters, J. C., \& Goebel, R. (2016). When the brain takes 'BOLD'steps: real-time fMRI neurofeedback can further enhance the 
ability to gradually self-regulate regional brain activation. Neuroscience.

Stalnaker, T. A., Calhoon, G. G., Ogawa, M., Roesch, M. R., \& Schoenbaum, G. (2012). Reward prediction error signaling in posterior dorsomedial striatum is action specific. Journal of Neuroscience, 32(30), 10296-10305.

Subramanian, L., Hindle, J. V., Johnston, S., Roberts, M. V., Husain, M., Goebel, R., \& Linden, D. (2011). Real-time functional magnetic resonance imaging neurofeedback for treatment of Parkinson's disease. Journal of Neuroscience, 31(45), 16309-16317.

Talairach, J., \& Tournoux, P. (1988). Co-planar stereotaxic atlas of the human brain. 3-Dimensional proportional system: an approach to cerebral imaging.

Tanaka, S. C., Balleine, B. W., \& O'Doherty, J. P. (2008). Calculating consequences: brain systems that encode the causal effects of actions. Journal of Neuroscience, 28(26), 6750-6755.

Tanaka, S. C., Doya, K., Okada, G., Ueda, K., Okamoto, Y., \& Yamawaki, S. (2004). Prediction of immediate and future rewards differentially recruits corticobasal ganglia loops. Nature neuroscience, 7(8), 887.

Tang, Y.-Y., Hölzel, B. K., \& Posner, M. I. (2015). The neuroscience of mindfulness meditation. Nature reviews neuroscience, 16(4), 213-225.

Tricomi, E. M., Balleine, B. W., \& O’Doherty, J. P. (2009). A specific role for posterior dorsolateral striatum in human habit learning. European Journal of Neuroscience, 29(11), 2225-2232.

Tricomi, E. M., Delgado, M. R., \& Fiez, J. A. (2004). Modulation of caudate activity by action contingency. Neuron, 41(2), 281-292.

Valentin, V. V., Dickinson, A., \& O'Doherty, J. P. (2007). Determining the neural substrates of goal-directed learning in the human brain. Journal of Neuroscience, 27(15), 4019-4026.

Weissman, D. H., Gopalakrishnan, A., Hazlett, C., \& Woldorff, M. (2004). Dorsal 
anterior cingulate cortex resolves conflict from distracting stimuli by boosting attention toward relevant events. Cerebral Cortex, 15(2), 229-237.

Young, K. D., Siegle, G. J., Zotev, V., Phillips, R., Misaki, M., Yuan, H., . . Bodurka, J. (2017). Randomized clinical trial of real-time fMRI amygdala neurofeedback for major depressive disorder: effects on symptoms and autobiographical memory recall. American Journal of Psychiatry, 174(8), 748-755.

Zilverstand, A., Sorger, B., Sarkheil, P., \& Goebel, R. (2015). fMRI neurofeedback facilitates anxiety regulation in females with spider phobia. Frontiers in behavioral neuroscience, 9, 148.

Zilverstand, A., Sorger, B., Slaats-Willemse, D., Kan, C. C., Goebel, R., \& Buitelaar, J. K. (2017). fMRI neurofeedback training for increasing anterior cingulate cortex activation in adult attention deficit hyperactivity disorder. An exploratory randomized, single-blinded study. PloS one, 12(1), e0170795.

\section{Acknowledgements}

The authors would like to thank Florian Krause for valuable theoretical input and gratefully acknowledge the support of the BrainGain Smart Mix Programme of the Netherlands Ministry of Economic Affairs and the Netherlands Ministry of Education, Culture and Science. This work was also supported by the Netherlands Organization for Scientific Research [NWO; RUBICON 446-09-010 to BS] and the European Research Council [ERC advanced grant \#269853 to RG]. 


Chapter 5 - Summary, General Discussion, Conclusion 



\section{Summary}

While the major focus of previous neurofeedback work lay on observing changes in the neurofeedback target or task-specific psychological outcome variables, only a much smaller part of research has been dedicated to study the general mechanisms of self-regulation and learning in the context of neurofeedback. To contribute to an understanding of how the brain responds to neurofeedback, this thesis focused on the mechanisms contributing to neurofeedback-guided self-regulation across different neurofeedback tasks and time.

In chapter 2, we focused on whether neurofeedback performance and psychological effects of neurofeedback are influenced by different training styles. Specifically, we investigated the effects of providing an initial neurofeedback task with assumed lower task demands before switching to the main training. Participants underwent three real-time fMRI neurofeedback-training sessions, in order to learn to regulate activation of the anterior insula (aINS) to three different target levels, either by performing the identical emotion regulation task over the whole training (constant training style group: CON), or by first training to reach three target levels with motor-imagery-driven neurofeedback from the supplementary motor area (SMA; changing training style group: $\mathrm{CHA}$ ). While achieving finegrained control over the fMRI-signal level was more feasible for participants receiving SMA in comparison to alNS neurofeedback during the initial training, both groups were able to significantly improve their alNS regulation performance over the subsequent sessions. Although both groups did not 
differ in their ability to stabilize the neurofeedback signal at a certain target level, the group initially obtaining neurofeedback experience through training with a motor-imagery task showed an increased tendency to upregulate the neurofeedback signal, which correlated with more effective training outcomes with regard to post-training positive affect. Findings were supported by participants' self-evaluation on personal neurofeedback regulation abilities and motivation. Overall, the results of chapter 2 support that neurofeedback guided emotion regulation is associated with subjective experiences of emotion. While differences between training styles are subtle, the overall pattern of group differences suggests that different training styles modulate self-regulation and psychological outcomes of a neurofeedback training.

In chapter 3, we focused on the neural basis of neurofeedback guided selfregulation and how it changes over time. By comparing brain activation between the two different mental tasks described in chapter 2 (SMA and aINS neurofeedback), we could replicate recent findings on involvement of a domain-general network in neurofeedback (Emmert et al., 2016) and in addition revealed differential activation in task-specific regions selectively involved in emotion regulation and motor imagery. Across both neurofeedback tasks, neurofeedback target regions showed significant increases in connectivity to the rest of the brain. Significant connectivity clusters of the two different target regions (SMA and aINS) overlapped with brain activation shared between both tasks, supporting the domain general function of the involved network. During the subsequent sessions of alNS 
neurofeedback training, activation remained largely constant, with a trend for increased striatum activation for the $\mathrm{CHA}$ group that was not present in the CON group. Connectivity between the target region and several cortical regions was significant during all sessions, particularly to regions that have been shown to be activated (lateral prefrontal, parietal, insular) or deactivated (hubs of the default mode network, DMN) across different neurofeedback tasks (Emmert et al., 2016). Additionally, connectivity between the target region and the anterior striatum was only observed during the last session. Overall, the results of this chapter support the notion, that during neurofeedback a defined network is recruited reliably. They suggest, that regions of this network synchronize their activation with the neurofeedback target, while especially the striatum could be implicated in learning effects in response to neurofeedback.

In chapter 4, we focused on the neural basis of feedback processing during neurofeedack guided self-regulation. For this matter, we compared fMRI activation across different mental tasks involving gradual self-regulation with and without providing neurofeedback. Participants freely chose one self-regulation task and underwent two training sessions, one with and one without receiving neurofeedback. Neurofeedback signals were provided based on activity in task-related, individually defined target regions. Wholebrain analysis revealed, that a network of cortical control regions as well as regions implicated in reward and feedback processing were activated during neurofeedback compared to rest. Self-regulation with feedback was accompanied by stronger activation within the striatum across different 
mental tasks. Additional time-resolved single-trial analysis revealed that neurofeedback performance was positively correlated with a delayed brain response in the striatum that reflected the accuracy of self-regulation.

\section{General Discussion}

\subsection{Task-general effects of neurofeedback}

The reliable activation of a network of prefrontal control areas (aINS, ventrolateral and dorsolateral prefrontal cortex (vl/dIPFC) and anterior cingulate cortex (ACC)) across various neurofeedback tasks (chapter 3 and 4, see also Emmert et al. (2016)) suggests that during neurofeedback a network is trained, which exerts control over a wide range of cognitive and affective processes. That the recruited network is involved in many different mental tasks suggests that it is not narrowly dependent on a particular task setting. It seems therefore likely that this network will exert its influence also in other mental tasks outside the neurofeedback setting. Studies on emotional self-regulation without neurofeedback support this notion: The aINS, VIPFC, dIPFC and ACC have been shown to be involved in endogenous generation of emotional states of positive as well as negative valance, and across different mental imagery modalities (Engen, Kanske, \& Singer, 2016). Additionally, the aINS, VIPFC, dIPFC and ACC appear to be crucial in downregulating negative emotions: Dörfel et al. (2014) could show that across emotional down-regulation strategies the dIPFC, ACC and aINS were reliably recruited, while VIPFC activation was particularly pronounced during reappraisal. The transfer from neurofeedback training effects in this network 
to clinically relevant forms of emotional self-regulation without neurofeedback seems therefore to be likely. In accordance, it recently has been shown that just one session of neurofeedback training can increase efficacy of mental strategies applied during cognitive-behavioral therapy in depressed patients (MacDuffie et al., 2018).

Considering the reliable involvement of the neurofeedback network described by Emmert in the here described work, it is fair to assume that the target-level paradigms used in this thesis as well as paradigms making use of only up- or down-regulation (described by Emmert and colleagues) recruit the same set of regions. In comparison to Emmert et al. (2016), the paradigms described in this thesis have also been associated with decreased activation in the pINS in comparison to baseline (chapter 3) and in comparison passive viewing of neurofeedback (chapter 4) across different mental tasks. Such deactivation of the pINS was present during emotionregulation neurofeedback (chapter 3 ) as well as during non-affective neurofeedback tasks (chapter 3 and 4).

While the consistency of results suggests the relevance of pINS deactivation in neurofeedback guided self-regulation, theoretical accounts for the underlying mechanism are at the moment hypothetical. The functional role of the pINS is multifaceted and various functions have been suggested (regarding the role of the pINS in pain processing see Apkarian, Bushnell, Treede, and Zubieta (2005), Segerdahl, Mezue, Okell, Farrar, and Tracey (2015), Davis, Bushnell, lannetti, Lawrence, and Coghill (2015); regarding its 
involvement in somatosensory and body representation see Tsakiris, Hesse, Boy, Haggard, and Fink (2006), Heydrich and Blanke (2013), Cereda, Ghika, Maeder, and Bogousslavsky (2002); for auditory processing and speech see Bamiou et al. (2006), Eickhoff, Heim, Zilles, and Amunts (2009), Wise, Greene, Büchel, and Scott (1999)).

Overall, more posterior sections of the insula contribute to various sensory processes, while medial-anterior sections are associated with emotions, executive functions and memory (Cloutman, Binney, Drakesmith, Parker, \& Ralph, 2012; A. D. Craig \& Craig, 2009; Kurth, Zilles, Fox, Laird, \& Eickhoff, 2010). It remains a matter of debate, which common function the pINS serves across different sensory modalities. A variety of roles have been suggested, including interoception, i.e., awareness of bodily functions (A. Craig, 2003; A. D. Craig, 2002; Kuehn, Mueller, Lohmann, \& SchuetzBosbach, 2016; Simmons et al., 2013), and encoding of sensory intensity (Davis et al., 2015; Moayedi \& Weissman-Fogel, 2009; Olausson et al., 2002; Seminowicz \& Davis, 2006). In the context of neurofeedback, deactivation of the pINS could reflect a high attentional focus on the ongoing mental imagery task, so that attentional weight on sensory input is reduced not to interfere with ongoing imagery. Accordingly, Wager, Spicer, Insler, and Smith (2014) describe pINS and DMN de-activation being reflective of task demand as well as predicting successful task performance during a working memory task, in accordance with the observed deactivation pattern described in chapter 3 and 4 . 


\subsection{Task-specific effects of neurofeedback}

Besides providing further evidence for task-general mechanisms of selfregulation in neurofeedback, the work presented in chapter 3 of this thesis also shows that areas specific to a certain mental task are recruited during neurofeedback. Comparing activation between emotion-regulation neurofeedback and motor-imagery neurofeedback showed spread out differences in activation and modulations in connectivity. However, it has to be noted that reported activation differences were acquired in a small sample and might reflect activation specific to participants in our sample. Nevertheless, it is promising that already on such a small scale neurofeedback tasks showed differing activation in task-relevant areas. For example, brainstem activation was only observed during motor-imagery neurofeedback and has previously been observed in motor imagery of running compared to mental imagery of lying (Jahn et al., 2008). This underlines the capability of neurofeedback to target a specific neural mechanism instead of general self-regulation skills.

Accordingly, recent neurofeedback studies have targeted the neural basis of very specific emotional states as affiliative emotions (Moll et al., 2014). In the future, such training schemes could help to treat very specific neuropsychiatric deficits with neurofeedback. For example, Green, Ralph, Moll, Deakin, and Zahn (2012) showed that self-blame in depression was related to alterations in functional coupling between the anterior temporal cortex and the subgenual cingulate cortex. Subsequently, Zahn et al. (2017) reported the possibility of modulating coupling between these regions 
through neurofeedback. By developing such specific neurofeedback tasks, neurofeedback can be tailored towards the specific symptoms of patients.

Differentiating between task-general and task-specific components of neurofeedback could also help to fine-tune the design of neurofeedback control groups, a topic of ongoing debate in the neurofeedback community: A recent study has for example shown that controlling an area responsive to visual scenes with visual imagery significantly improved depression symptoms of patients, with effect size comparable to symptom improvements after emotion-regulation neurofeedback and exceeding the expected effect size of placebo effects (Mehler et al., 2018). Understanding which neural and psychological mechanisms are shared between different types of neurofeedback could enable researchers to take the expected shared effects of different mental tasks into account for choosing control tasks or determining expected effect sizes of group differences.

\subsection{Limitations and future directions}

While the presented findings extend the field by providing novel insights into the working mechanisms of neurofeedback, future studies have to determine to which extend they can be generalized to other neurofeedback settings. Several aspects of the presented work potentially limit the generalizability of our findings. Overall, the presented studies are based on rather small samples. Nonetheless, whole-brain results based on pooled data of mental tasks and groups (chapters 3 and 4) were in accordance with 
previous research (Emmert et al., 2016) and appeared to be replicable over the studies described in this thesis. In turn, especially the sub-parts of the here presented work that focus on specific effects of neurofeedback tasks (chapters 2 and 3) should be reproduced with larger samples. Despite including different neurofeedback tasks and neurofeedback target regions, the here described research relies on several design properties, that potentially limit generalizability of results towards other neurofeedback studies:

Firstly, the applied neurofeedback tasks required participants to reach and hold the neurofeedback signal at defined target activation levels. As this includes up-regulating activation in a region, as well as down-regulating (when exceeding the target level), it remains open, whether continuous training of only up-regulation or only down-regulation will lead to different training outcomes.

Secondly, the majority of neurofeedback applications is based on electroencephalography (EEG). EEG neurofeedback can be updated in milliseconds, as it is not affected by the hemodynamic delay. This might entail, that mental operations performed during EEG neurofeedback might differ from mental operations performed during $\mathrm{FMRI}$ neurofeedback. For example, working memory capacities might be used differently as EEG neurofeedback does not constantly have to be related to a mental operation that occurred 6-8 seconds backwards in time. Indeed, Perronnet and colleagues describe more BOLD activation during motor-imagery-guided by 
fMRI-neurofeedback than during EEG-neurofeedback in comparison to rest, and most pronounced activation during bimodal neurofeedback, i.e., were EEG- and fMRI-neurofeedback information is integrated (Perronnet et al., 2017). Specifically, areas beyond the motor system, including the IFG and the aINS, were only recruited when incorporating fMRI information, suggesting a modulatory influence on the task-general network.

Thirdly, participants performed active self-regulation: They were aware that they were receiving neurofeedback and aimed to achieve control over the neurofeedback signal. While most neurofeedback studies (including the experiments described in this thesis) require voluntary self-regulation involving the awareness of the participant to be provided with neurofeedback, the alternative covert-neurofeedback approach relies on sub-conscious associative learning (Koizumi et al., 2017; Ramot, Grossman, Friedman, \& Malach, 2016; Taschereau-Dumouchel, Liu, \& Lau, 2018). Although taking care that participants were not aware of receiving neurofeedback, Ramot and colleagues described increasing connectivity between the target ROI and the default-mode network, cingulate cortex, thalamus, striatum, brainstem, and cerebellum during covert neurofeedback over time, as well as increased activation in the lateral fissure, ACC and striatum in response to neurofeedback (Ramot et al., 2016). Notably, profound involvement of prefrontal and parietal control areas was not detected. This lack of control network involvement in comparison to the findings described in this thesis (chapter 3 and 4) supports that a core network of regions is reactive to neurofeedback, which is extended by 
prefrontal and parietal control regions when (voluntary) self-regulation is involved. Future research could determine whether covert neurofeedback paradigms that do not require demanding self-regulatory skills could be used for improving the efficacy of neurofeedback paradigms, or whether they are better suited for a certain patient population with deficits in the circuitry of self-regulation.

\section{Conclusion}

Overall, the research summarized in this thesis sheds light on several working mechanisms of neurofeedback by investigating involved psychological processes (e.g. self-efficacy and positive affect after emotionregulation neurofeedback training), by describing brain systems generally recruited during neurofeedback guided self-regulation, by describing brain systems recruited during specific neurofeedback tasks, as well as showing functional changes that the brain undergoes during neurofeedback over time, and by revealing processing of neurofeedback information in the striatum, as a key region in feedback learning.

Taken together, these findings support that neurofeedback affects the brain by stimulating the reward system, while simultaneously guiding higher-level cognitive control and task specific core networks. The reliable interplay of multiple regions thereby provides a pronounced opportunity for inducing changes on a network level, as during neurofeedback an extensive network of regions potentially synchronizes with the target region. In addition to the 
implicated clinical possibilities, neurofeedback thereby provides a controlled setting to study how brain networks adapt in response to feedback. Considering that most neural systems function through dynamic interactions between neural activity and intrinsic as well as environmental feedback, neurofeedback constitutes a holistic research tool for neuroscience. 


\section{References}

Apkarian, A. V., Bushnell, M. C., Treede, R.-D., \& Zubieta, J.-K. (2005). Human brain mechanisms of pain perception and regulation in health and disease. European journal of pain, 9(4), 463-484.

Bamiou, D.-E., Musiek, F., Stow, I., Stevens, J., Cipolotti, L., Brown, M., \& Luxon, L. (2006). Auditory temporal processing deficits in patients with insular stroke. Neurology, 67(4), 614-619.

Cereda, C., Ghika, J., Maeder, P., \& Bogousslavsky, J. (2002). Strokes restricted to the insular cortex. Neurology, 59(12), 1950-1955.

Cloutman, L. L., Binney, R. J., Drakesmith, M., Parker, G. J., \& Ralph, M. A. L. (2012). The variation of function across the human insula mirrors its patterns of structural connectivity: evidence from in vivo probabilistic tractography. Neurolmage, 59(4), 3514-3521.

Craig, A. (2003). Interoception: the sense of the physiological condition of the body. Current opinion in neurobiology, 13(4), 500-505.

Craig, A. D. (2002). How do you feel? Interoception: the sense of the physiological condition of the body. Nature reviews neuroscience, 3(8), 655.

Craig, A. D., \& Craig, A. (2009). How do you feel--now? The anterior insula and human awareness. Nature reviews neuroscience, 10(1).

Davis, K. D., Bushnell, M. C., lannetti, G. D., Lawrence, K. S., \& Coghill, R. (2015). Evidence against pain specificity in the dorsal posterior insula. F1000Research, 4.

Dörfel, D., Lamke, J.-P., Hummel, F., Wagner, U., Erk, S., \& Walter, H. (2014). Common and differential neural networks of emotion regulation by detachment, reinterpretation, distraction, and expressive suppression: a comparative fMRI investigation. Neurolmage, 101, 298-309.

Eickhoff, S. B., Heim, S., Zilles, K., \& Amunts, K. (2009). A systems perspective on the 
effective connectivity of overt speech production. Philosophical Transactions of the Royal Society of London A: Mathematical, Physical and Engineering Sciences, 367(1896), 2399-2421.

Emmert, K., Kopel, R., Sulzer, J., Brühl, A. B., Berman, B. D., Linden, D. E., . . Frank, S. (2016). Meta-analysis of real-time fMRI neurofeedback studies using individual participant data: How is brain regulation mediated? Neurolmage, $124,806-812$.

Engen, H. G., Kanske, P., \& Singer, T. (2016). The neural component-process architecture of endogenously generated emotion. Social cognitive and affective neuroscience, 12(2), 197-211.

Green, S., Ralph, M. A. L., Moll, J., Deakin, J. F., \& Zahn, R. (2012). Guilt-selective functional disconnection of anterior temporal and subgenual cortices in major depressive disorder. Archives of General Psychiatry, 69(10), 10141021.

Heydrich, L., \& Blanke, O. (2013). Distinct illusory own-body perceptions caused by damage to posterior insula and extrastriate cortex. Brain, 136(3), 790-803.

Jahn, K., Deutschländer, A., Stephan, T., Kalla, R., Wiesmann, M., Strupp, M., \& Brandt, T. (2008). Imaging human supraspinal locomotor centers in brainstem and cerebellum. Neurolmage, 39(2), 786-792.

Koizumi, A., Amano, K., Cortese, A., Shibata, K., Yoshida, W., Seymour, B., .. . Lau, H. (2017). Fear reduction without fear through reinforcement of neural activity that bypasses conscious exposure. Nature Human Behaviour, 1(1), 0006.

Kuehn, E., Mueller, K., Lohmann, G., \& Schuetz-Bosbach, S. (2016). Interoceptive awareness changes the posterior insula functional connectivity profile. Brain Structure and Function, 221(3), 1555-1571.

Kurth, F., Zilles, K., Fox, P. T., Laird, A. R., \& Eickhoff, S. B. (2010). A link between the 
systems: functional differentiation and integration within the human insula revealed by meta-analysis. Brain Structure and Function, 214(5-6), 519-534. MacDuffie, K. E., Maclnnes, J., Dickerson, K. C., Eddington, K. M., Strauman, T. J., \& Adcock, R. A. (2018). Single session real-time fMRI neurofeedback has a lasting impact on cognitive behavioral therapy strategies. Neurolmage: Clinical, 19, 868-875.

Mehler, D. M., Sokunbi, M. O., Habes, I., Barawi, K., Subramanian, L., Range, M., . . . Keedwell, P. (2018). Targeting the affective brain-a randomized controlled trial of real-time fMRI neurofeedback in patients with depression. Neuropsychopharmacology, 1.

Moayedi, M., \& Weissman-Fogel, I. (2009). Is the insula the "how much" intensity coder? Journal of neurophysiology, 102(3), 1345-1347.

Moll, J., Weingartner, J. H., Bado, P., Basilio, R., Sato, J. R., Melo, B. R., . . Zahn, R. (2014). Voluntary enhancement of neural signatures of affiliative emotion using FMRI neurofeedback. PloS one, 9(5), e97343.

Olausson, H., Lamarre, Y., Backlund, H., Morin, C., Wallin, B., Starck, G., . . Vallbo, A. (2002). Unmyelinated tactile afferents signal touch and project to insular cortex. Nature neuroscience, 5(9), 900.

Perronnet, L., Lécuyer, A., Mano, M., Bannier, E., Lotte, F., Clerc, M., \& Barillot, C. (2017). Unimodal versus bimodal EEG-fMRI neurofeedback of a motor imagery task. Frontiers in human neuroscience, 11, 193.

Ramot, M., Grossman, S., Friedman, D., \& Malach, R. (2016). Covert neurofeedback without awareness shapes cortical network spontaneous connectivity. Proceedings of the National Academy of Sciences, 113(17), E2413-E2420.

Riecker, A., Ackermann, H., Wildgruber, D., Dogil, G., \& Grodd, W. (2000). Opposite hemispheric lateralization effects during speaking and singing at motor cortex, insula and cerebellum. Neuroreport, 11(9), 1997-2000. 
Segerdahl, A. R., Mezue, M., Okell, T. W., Farrar, J. T., \& Tracey, I. (2015). The dorsal posterior insula subserves a fundamental role in human pain. Nature neuroscience, 18(4), 499.

Seminowicz, D. A., \& Davis, K. D. (2006). Interactions of pain intensity and cognitive load: the brain stays on task. Cerebral Cortex, 17(6), 1412-1422.

Simmons, W. K., Avery, J. A., Barcalow, J. C., Bodurka, J., Drevets, W. C., \& Bellgowan, P. (2013). Keeping the body in mind: insula functional organization and functional connectivity integrate interoceptive, exteroceptive, and emotional awareness. Human Brain Mapping, 34(11), 2944-2958.

Taschereau-Dumouchel, V., Liu, K.-y., \& Lau, H. (2018). Unconscious psychological treatments for physiological survival circuits. Current Opinion in Behavioral Sciences, 24, 62-68.

Tsakiris, M., Hesse, M. D., Boy, C., Haggard, P., \& Fink, G. R. (2006). Neural signatures of body ownership: a sensory network for bodily self-consciousness. Cerebral Cortex, 17(10), 2235-2244.

Wager, T. D., Spicer, J., Insler, R., \& Smith, E. E. (2014). The neural bases of distracterresistant working memory. Cognitive, Affective, \& Behavioral Neuroscience, 14(1), 90-105.

Wise, R., Greene, J., Büchel, C., \& Scott, S. K. (1999). Brain regions involved in articulation. The Lancet, 353(9158), 1057-1061.

Zahn, R., Weingartner, J., Basilio, R., Bado, P., Mattos, P., de Oliveira-Souza, S. J., . . . Moll, J. (2017). 30 Blame rebalance fmri feedback proof-of-concept trial in major depressive disorder: BMJ Publishing Group Ltd. 


Appendix 



\section{Knowledge Valorisation}

In the last fifty years, a sharp rise in mental health issues has been observed (Kim, Coumar, Lober, \& Kim, 2011; Whitaker, 2005), widely described as the „mentalhealth epidemic". During the same time, technical improvements like the development of functional magnetic resonance imaging and the exponential growth of processing speed and digital memory has put neuroscience, psychology and psychiatry into the state to create mental-health treatments based on excessively growing data related to human (e.g., brain) functions.

While a research community contributing to such a massive increase of clinically relevant data has the ethical responsibility to support the utilization of its research in the clinical practice, developing treatments based on fast-changing and costintensive neuroimaging techniques remains a challenge under constant economic pressure. Increasing the efficacy of treatments and tailoring it to the patient population that will benefit the most from it will help to optimally translate findings from basic research in cognitive, affective and social neuroscience to the clinical domain.

In the process of this transition, neurofeedback has to compete or merge with other mental-health treatments that do not require such specialized technical equipment and are easier to access, as psychopharmacological treatments, psychotherapy or mindfulness-based approaches. Currently, research that observes such interactions between rtfMRI and other mental health treatments is emerging. A recent study could demonstrate that neurofeedback can support cognitive behavioural therapy by improving meta-cognitive abilities of participants (MacDuffie et al., 2018). An ongoing clinical trial is also evaluating whether brain activation during 
neurofeedback can function as a biomarker for responsiveness to pharmacological treatments (Peciña, 2016). However, potential interaction with regard to the neural effects of such combinations are hard to predict, due to complexity of the involved neural mechanisms.

Acquiring and analyzing data of the neural processes that take place during neurofeedback is therefore crucial, in order to form valid hypothesis on how other available treatment approaches can contribute to the effects of neurofeedback and vice versa. Especially combinations between neurofeedback and brain stimulation techniques could constitute a first pathway beyond neurofeedback-only interventions, as both techniques provide flexible control over neural treatment effects. But while first closed-loop neurofeedback transcranial magnetic stimulation (NF-TMS) systems have already successfully been implemented (Koganemaru et al., 2018; Sokhadze et al., 2014), their applicability in the clinical domain is still in its infancy.

Another group of treatments that shows potential for being combined with neurofeedback are biofeedback trainings that do not feedback brain activation, but rather respiratory or cardiac activity. Such biofeedback approaches, as heart rate variability biofeedback, have also shown to constitute effective treatment options for major psychiatric disorders (see Siepmann, Aykac, Unterdörfer, Petrowski, and Mueck-Weymann (2008); Karavidas et al. (2007)), and could contribute to the training of self-regulation strategies, interoceptive abilities, as well as self-efficacy (Gevirtz, 2013).

In addition, meditation based treatments share several psychological core aspects with neurofeedback interventions, as training of self-regulation skills and 
introspection (Baird, Mrazek, Phillips, \& Schooler, 2014; Tang et al., 2007; Tang, Posner, \& Rothbart, 2014). As these approaches are not as cost-intensive as fMRINF approaches, but potentially require more training sessions, combining such interventions with neurofeedback could provide a possibility to allow for continuous self-regulation training, while reducing treatment costs.

Another option for reducing treatment costs would be the application of cheaper and more easily accessible neuroimaging techniques for generating neurofeedback, as electroencephalography (EEG) or functional near-infrared spectroscopy (fNIRS). While these techniques lack the ability to provide reliable neurofeedback from subcortical areas, they are not associated with so many practical issues as MRI (e.g. related to safety) and can also be used without trained technical staff: Contemporary developments as BCI-EEG devices for home use (Sellers, Vaughan, \& Wolpaw, 2010) could therefor help to integrate neurofeedback into the daily life of patients. Understanding how participants react to different types of neurofeedback will contribute to sensible decisions on which neuroimaging techniques are best to be applied for certain clinical conditions.

But before understanding the unique effects of a particular neurofeedback setting, as well as the effects emerging from combinations between neurofeedback with other therapies, research on the neural basis of neurofeedback is a first, necessary step. Neurofeedback can only develop its full potential as a clinical tool when we understand how it works.

To achieve this 
i. ...improvements of training designs as described in chapter 2 and 3 of this thesis will help to increase the cost efficacy of neurofeedback. At the same time they provide an example for transfer of self-regulation skills and thus give an optimistic outlook for more holistic training schemes, which not only implement different neurofeedback tasks, but in consecutive treatment phases apply neurofeedback and other mental health interventions.

ii. ...research on the neural mechanisms of neurofeedback (as described in chapter 3 and 4), will allow to understand which properties of neurofeedback are unique in comparison to other types of treatment. Especially when considering that striatum activation (as described in chapter 4) could be modulated directly at reward sensitive time windows with neurofeedback-guided neurostimulation, neurofeedback contains powerful potential for reshaping dysfunctional brain circuits through reinforcement learning.

With its potential for integrating state of the art research from neuroscience, psychiatry and psychology into one comprehensive treatment approach, neurofeedback constitutes a modern treatment for psychiatric conditions that manifest themselves in the brain as well as in psychological experiences. 


\section{References}

Baird, B., Mrazek, M. D., Phillips, D. T., \& Schooler, J. W. (2014). Domain-specific enhancement of metacognitive ability following meditation training. Journal of Experimental Psychology: General, 143(5), 1972.

Gevirtz, R. (2013). The promise of heart rate variability biofeedback: Evidencebased applications. Biofeedback, 41(3), 110-120.

Karavidas, M. K., Lehrer, P. M., Vaschillo, E., Vaschillo, B., Marin, H., Buyske, S., . . . Hassett, A. (2007). Preliminary results of an open label study of heart rate variability biofeedback for the treatment of major depression. Applied psychophysiology and biofeedback, 32(1), 19-30.

Kim, E.-H., Coumar, A., Lober, W. B., \& Kim, Y. (2011). Addressing mental health epidemic among university students via web-based, self-screening, and referral system: a preliminary study. IEEE Transactions on Information Technology in Biomedicine, 15(2), 301-307.

Koganemaru, S., Mikami, Y., Maezawa, H., Ikeda, S., Ikoma, K., \& Mima, T. (2018). Neurofeedback Control of the Human GABAergic System Using Noninvasive Brain Stimulation. Neuroscience, 380, 38-48.

MacDuffie, K. E., MacInnes, J., Dickerson, K. C., Eddington, K. M., Strauman, T. J., \& Adcock, R. A. (2018). Single session real-time fMRI neurofeedback has a lasting impact on cognitive behavioral therapy strategies. Neurolmage: Clinical, 19, 868-875.

Peciña, M. (2016). Study of Neural Responses Induced by Antidepressant Effects (SONRISA). Identification No. NCT02674529. Retrieved from https://clinicaltrials.gov/ct2/show/NCT02674529.

Sellers, E. W., Vaughan, T. M., \& Wolpaw, J. R. (2010). A brain-computer interface for long-term independent home use. Amyotrophic lateral sclerosis, 11(5), 449-455. 
Siepmann, M., Aykac, V., Unterdörfer, J., Petrowski, K., \& Mueck-Weymann, M. (2008). A pilot study on the effects of heart rate variability biofeedback in patients with depression and in healthy subjects. Applied psychophysiology and biofeedback, 33(4), 195-201.

Sokhadze, E. M., El-Baz, A. S., Tasman, A., Sears, L. L., Wang, Y., Lamina, E. V., \& Casanova, M. F. (2014). Neuromodulation integrating rTMS and neurofeedback for the treatment of autism spectrum disorder: an exploratory study. Applied psychophysiology and biofeedback, 39(3-4), 237257.

Tang, Y., Ma, Y., Wang, J., Fan, Y., Feng, S., Lu, Q., . . Fan, M. (2007). Short-term meditation training improves attention and self-regulation. Proceedings of the National Academy of Sciences, 104(43), 17152-17156.

Tang, Y., Posner, M. I., \& Rothbart, M. K. (2014). Meditation improves self-regulation over the life span. Annals of the New York Academy of Sciences, 1307(1), 104-111.

Whitaker, R. (2005). Anatomy of an epidemic: Psychiatric drugs and the astonishing rise of mental illness in America. Ethical Human Sciences and Services, 7(1), 23-35. 


\section{Acknowledgements}

I would like to take this opportunity to thank all the people that actively contributed to the conducted research and my development in the field of neuroscience over the last years. I am especially grateful to my supervisors Rainer and Bettina, who provided me with the opportunity and means to develop my own scientific ideas and made it possible for me to connect with the Braintrain consortium and the Hemodynamic $\mathrm{BCl}$ group.

Undoubtedly Carmine, Armin, Rick, Florian, Michael, Judith, Maria, Panda, Makis, Anita, Caroline, Claudia, Bianca, Ralph, Quentin and Hester added a lot to my work (especially cake) during hours of conversation shared over quality coffee. All members of Brain Innovation and Braintrain contributed with their various technical expertise and personalities as well as Amaia, Laurien, Tabea, Hannah and all other members of the Hemodynamic $\mathrm{BCl}$ group. Special thanks I want to dedicate to the core members of the lunch consortium, for constantly high humorous input during constantly low culinary experiences: Carmine, Rick (and Florian and Caroline and Michael...); Carmine also for compensating everything with proper Italian food and for being one of my earliest office mates, as was Armin, the unmatched movie database, Dr. Lumma, who constantly spread enlightenment, and Dr. Hoffmann, who contributed gourmet juice.

Finally, I want to thank all my friends for being so understanding towards my stoic work ethic in the last years, and my parents for their intellectual and practical contributions to my development. I cannot appreciate their support during these densely productive years enough. 



\section{Curriculum Vitae}

Leon Skottnik was born in Marl, Germany, on 10th August 1988 and graduated from Albert-Schweitzer-Gymnasium, Marl in 2007. He studied Philosophy and Dutch Studies from 2007 to 2008 at the University of Münster in Germany, Psychology at Maastricht University from 2008 to 2011 and Biology and Neuropsychology at the Open University of the Netherlands in Heerlen from 2009 to 2010. In 2011 he participated in the Erasmus program with which he studied Cognitive Neuroscience and Neuroscience of Language at Bangor University in the United Kingdom.

After obtaining his Bachelor of Science in Psychology from Maastricht University with merit, he followed the Research Master program in Cognitive and Clinical Neuroscience at Maastricht University from 2011 to 2013. During this time, he worked as a teaching assistant for neuroanatomy at Maastricht University and conducted his research master project on cortical thickness alterations induced by long-term meditation at the Department of Social Neuroscience of the Max Planck Institute for Human Cognitive and Brain Sciences in Leipzig, Germany. After receiving his Master of Science from Maastricht University, he continued his scientific work at the Department of Social Neuroscience in Leipzig focusing on neural mechanisms of emotion regulation and resilience until 2014.

In 2015 he started his PhD at the Department of Cognitive Neuroscience in the Faculty of Psychology and Neuroscience at Maastricht University and at Brain Innovation BV, Maastricht, the Netherlands. In 2018 he transitioned to the Department of Psychiatry and Neuropsychology at the Faculty of Health, Medicine and Life Science at Maastricht University, where his scientific work focuses on clinical applications of neurofeedback. 



\section{Publications}

\section{Publications in peer reviewed journals}

Skottnik, L., \& Linden, D. (2019). Mental imagery and brain regulation - new links between psychotherapy and neuroscience. Frontiers in Psychiatry, 10, 779 .

Skottnik, L., Sorger, B., Kamp, T., Linden, D., \& Goebel, R. (2019). Success and failure of controlling the real-time functional magnetic resonance imaging neurofeedback signal are reflected in the striatum. Brain and Behavior, 9(3), e01240.

Engen, H., Bernhardt, B., Skottnik, L., Ricard, M., \& Singer, T. (2018). Structural changes in socio-affective networks: Multi-modal MRI findings in long-term meditation practitioners. Neuropsychologia, 116, 26-33.

Skottnik, L. (2013). The function of the medial prefrontal cortex in emotions and empathy. Maastricht Student Journal of Psychology and Neuroscience, 1(1).

\section{Book chapters}

Skottnik, L., \& Linden, D. (in press). FMRI neurofeedback as treatment for depression. In S. Selvaraj, J. Soares, \& P. Brambilla (Eds.), Mood Disorders: Brain imaging and Therapeutic Implications. Cambridge: Cambridge University Press.

\section{Conference presentations}

Skottnik, L., Sorger, B., Benjamins, C., \& Goebel, R. (2019). Two tasks are better than one: Initial motor imagery neurofeedback can increase efficacy of rtfMRI neurofeedback-guided emotion regulation trainings. Poster session presented at the Real-time functional imaging and neurofeedback conference (rtFIN), Maastricht, The Netherlands. 
Mehler, D., Skottnik, L., Playle, R., Havermans, R., Schruers, K., Delespault, P., Goebel, R., \& Linden, D (2019). Novel trial design for fMRI neurofeedback using a Bayesian sampling plan: Evaluating neurofeedback as add-on treatment of depression. Poster session presented at the Real-time functional imaging and neurofeedback conference (rtFIN), Maastricht, The Netherlands.

Cox, M., Skottnik, L., Lührs, M., McNamara, R., Playle, R., Hood, K., Watson, G., Whittaker, J., Williams, A., Sakhuja, R., Ihssen, N., Linden, D., \& Subramanian L. (2019). Neurofeed back Training for Preventing Relapse to Alcohol Dependence. Talk presented at the International Conference on Psychological Sciences (ICPS), Paris, France.

Skottnik, L., Sorger, B., Kamp, T., \& Goebel, R. (2017). Involvement of the reward system during rtfMRI neurofeedback across various self-regulation tasks. Poster session presented at the Real-time functional imaging and neurofeedback conference (rtFIN), Nara, Japan.

Skottnik, L., (2017). Beyond up- and down-regulation: Learning to regulate brain activity to precise target levels. Talk presented at the Real-time functional imaging and neurofeedback conference 2017 (rtfin2017), Nara, Japan.

Skottnik, L., Sorger, B., Esposito, F., \& Goebel, R. (2015). Connectivity alterations of cognitive-control regions during fMRI-based neurofeed back training. Poster session presented at TALK 2 UR BRAIN - Advances in Neurofeedback: Research and Practice, Tel Aviv University, Ramat Aviv, Israel.

Skottnik, L., Bernhardt, B. , Engen, H. Valk, S., Ricard, M., \& Singer, T. (2014). Expert compassion and loving-kindness meditators show cortical thickness increases in socio-affective brain networks. Poster session presented at the 20th Annual Meeting of the Organization for Human Brain Mapping, Hamburg, Germany.

Abrahams, J., van der Bij, I., Brakelé, R., van Kuijk, S., Morawietz, A., Skottnik, L., Hausfeld, L. \& Santoro, L. (2010). What changes when the key changes? Talk presented at the Annual Psychology Student Research Meeting Maastricht, Maastricht, The Netherlands. 